Florida International University FIU Digital Commons

$4-1-2011$

\title{
Color-Based Surface Reflectance Separation for Scene Illumination Estimation and Rendering
}

Mouncef Lahlou

Florida International University, mlahl002@fiu.edu

DOI: $10.25148 /$ etd.FI1 1042702

Follow this and additional works at: https://digitalcommons.fiu.edu/etd

\section{Recommended Citation}

Lahlou, Mouncef, "Color-Based Surface Reflectance Separation for Scene Illumination Estimation and Rendering" (2011). FIU Electronic Theses and Dissertations. 381.

https://digitalcommons.fiu.edu/etd/381 


\title{
FLORIDA INTERNATIONAL UNIVERSITY
}

Miami, Florida

\section{COLOR-BASED SURFACE REFLECTANCE SEPARATION FOR SCENE \\ ILLUMINATION ESTIMATION AND RENDERING}

\author{
A dissertation submitted in partial fulfillment of the \\ requirements for the degree of \\ DOCTOR OF PHILOSOPHY \\ in \\ ELECTRICAL ENGINEERING \\ by \\ Mouncef Lahlou
}

2011 
To: Dean Amir Mirmiran

College of Engineering and Computing

This dissertation, written by Mouncef Lahlou, and entitled Color-Based Surface Reflectance Separation for Scene Illumination Estimation and Rendering, having been approved in respect to style and intellectual content, is referred to you for judgment.

We have read this dissertation and recommend that it be approved.

$\begin{array}{r}\text { Armando Barreto } \\ \hline \text { Jean Andrian } \\ \hline \text { Naphtali Rishe } \\ \hline \text { Malek Adjouadi, Major Professor }\end{array}$

Date of Defense: April 1, 2011

The dissertation of Mouncef Lahlou is approved.

Dean Amir Mirmiran
College of Engineering and Computing

Florida International University, 2011 
(C) Copyrights 2011 by Mouncef Lahlou All rights reserved. 


\section{DEDICATION}

To my marvelous parents who are always supportive in attaining my wishes and dreams, all my familly members for their unconditioned support and love, and my friends whom I had the pleasure to share several exceptional moments during the past years. 


\section{ACKNOWLEDGMENTS}

As humans, at one point in our life, we decide and become determined to achieve certain goals, which for some represent dreams and for others obligations that need to be fulfilled regardless of any kind of motivations. But most of us forget the true meaning of these achievements. We all seek a better living by advancing our knowledge mainly to help our society and facilitate our existence in this world. Knowledge in reality won't match the divine creation of something as small as an ant or as little as a fly, even after combining the knowledge of all humans and other living-beings that God has created throughout time.

First and foremost, all praises, acknowledgements, and thanks go to the Lord of the seven heavens and earth, lord of the great and supreme throne, ALLAH (s.w.t.) almighty, above all his creations. He is the one who gave me life, health, strength, wealth, knowledge and blessed me with countless of other bounties in all aspects, "Al-Hamdu wa Shukru li-Llah". His blessings have been endless and with his grace my wishes have always been fulfilled in a better way than I could have imagined them happening. Moreover, I would not be fully admitting my gratitude towards my creator, the merciful and the all-knowing, if I didn't recognize the people who have helped me during my years of study, I acknowledge their help without any adornments by simply following the teaching of one

of my beloved prophets, Muhammad (peace be upon him), who plainly said: "Any one who doesn't thank people has not thanked ALLAH".

I start by thanking my wonderful parents, with their care and love they have succeeded in planting in me the seeds of love for learning and helping others by easing their hardships through this life. Seeds that with time have flourished for me to understand the true 
meanings of this life, to not just satisfy our desires but rather to adhere to the commands of our creator with the hope to reach his mercy and gain the best life of the hereafter. Luckily, many people who I was fortunate to meet have contributed to the growth of these seeds in an excellent way. I was very fortunate to meet my advisor, mentor, and major professor Dr. Malek Adjouadi who during the past years has truly contributed to the person I'm today. Not only with his remarkable ways of transferring his knowledge to his students but also by teaching us leadership, motivation, and how to successfully attain and achieve our objectives. The care that he has always shown for our success in addition to his patience and trust has always pushed us to work harder to reach our goals. I sincerely appreciate his support during these past years, and thank him for always showing me the same level of care and help to succeed as if I was part of his family. My fellow CATE members were also of significant help during my graduate studies. I am appreciative for the many hours of meetings and brainstorming with Dr. Melvin Ayala and Dr. Mercedes Cabrerizo, during the final stages of this dissertation, and Dr. Magno R. Guillen during the early stages of my graduate projects within the CATE center. During my years in South Florida, I had the pleasure of meeting several people and some of them have left a lasting impression on me, not only because of their friendship but also for always considering me as one of their family members. I need to mention Shamim Khan's family, with their care and love I always felt being at home as soon as I entered their house and shared many moments with them. Also, a wonderful person who I was truly fortunate to meet and who became one of my excellent friends within a few years, Ana M. Guzman, she has always been there in my ups and downs with her magnificent care and love, not only for me but also for all her friends which makes her a very unique 
and precious person. Finally, I thank my committee members for their comments and suggestions during the beginning and final stages of this research. Last but not least, the support provided by the National Science Foundation through grants CNS-0959985, HDR-0833093, and CNS-1042341 was very well appreciated. I'm also thankful for the support provided by the FIU Graduate School through the Dissertation Year Fellowship received in my last year as a Ph.D. student. 


\title{
ABSTRACT OF THE DISSERTATION \\ COLOR-BASED SURFACE REFLECTANCE SEPARATION FOR SCENE ILLUMINATION ESTIMATION AND RENDERING
}

\author{
by \\ Mouncef Lahlou \\ Florida International University, 2011 \\ Miami, Florida \\ Professor Malek Adjouadi, Major Professor
}

Given the importance of color processing in computer vision and computer graphics, estimating and rendering illumination spectral reflectance of image scenes is important to advance the capability of a large class of applications such as scene reconstruction, rendering, surface segmentation, object recognition, and reflectance estimation. Consequently, this dissertation proposes effective methods for reflection components separation and rendering in single scene images. Based on the dichromatic reflectance model, a novel decomposition technique, named the Mean-Shift Decomposition (MSD) method, is introduced to separate the specular from diffuse reflectance components. This technique provides a direct access to surface shape information through diffuse shading pixel isolation. More importantly, this process does not require any local color segmentation process, which differs from the traditional methods that operate by aggregating color information along each image plane.

Exploiting the merits of the MSD method, a scene illumination rendering technique is designed to estimate the relative contributing specular reflectance attributes of a scene image. The image feature subset targeted provides a direct access to the surface 
illumination information, while a newly introduced efficient rendering method reshapes the dynamic range distribution of the specular reflectance components over each image color channel. This image enhancement technique renders the scene illumination reflection effectively without altering the scene's surface diffuse attributes contributing to realistic rendering effects.

As an ancillary contribution, an effective color constancy algorithm based on the dichromatic reflectance model was also developed. This algorithm selects image highlights in order to extract the prominent surface reflectance that reproduces the exact illumination chromaticity. This evaluation is presented using a novel voting scheme technique based on histogram analysis.

In each of the three main contributions, empirical evaluations were performed on synthetic and real-world image scenes taken from three different color image datasets. The experimental results show over $90 \%$ accuracy in illumination estimation contributing to near real world illumination rendering effects. 


\section{TABLE OF CONTENTS}

CHAPTER

PAGE

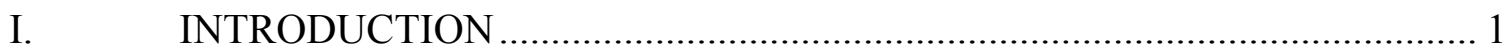

$1.1 \quad$ General Statement of the Problem Area ........................................................... 1

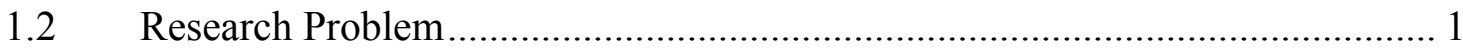

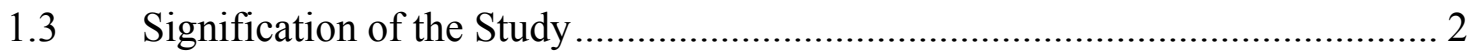

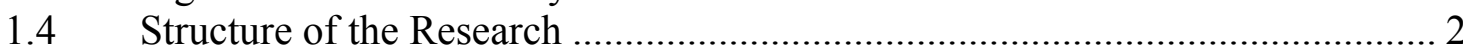

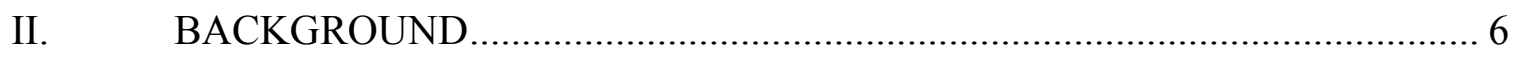

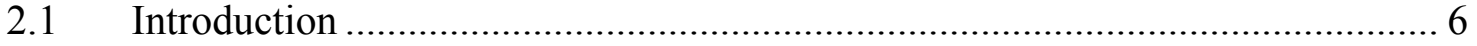

2.2 Illumination Spectral Power Distribution.................................................. 7

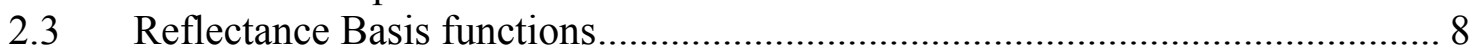

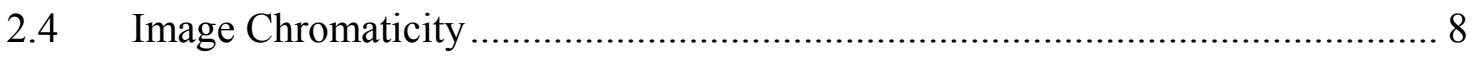

III. SURFACE REFLECTANCE COMPONENTS SEPARATION FROM SINGLE COLOR IMAGES USING THE MEAN-SHIFT DECOMPOSITION

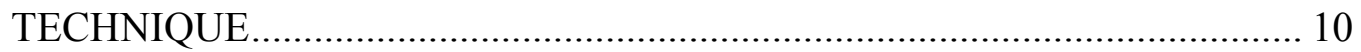

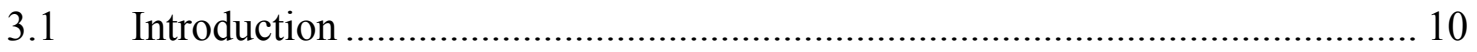

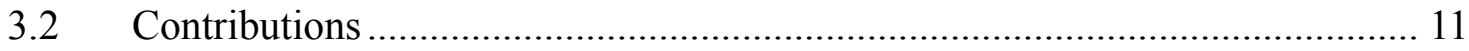

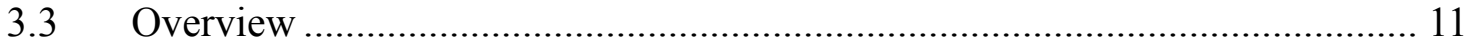

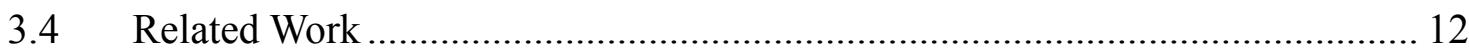

3.5 Reflection Model and Image Formation ..................................................... 13

3.6 Image-Chromaticity Color Space .............................................................. 16

3.7 Image Reflectance Components Classification ............................................... 18

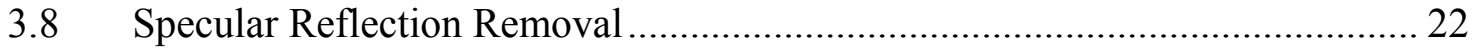

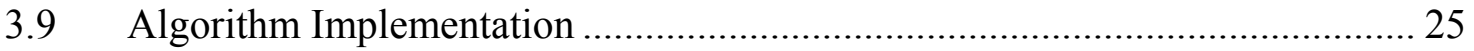

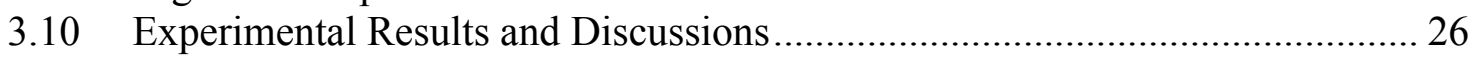

IV. COLOR BASED SPECULAR REFLECTANCE ESTIMATION FOR SCENE ILLUMINATION RENDERING ....................................... 34

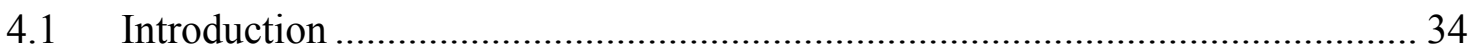

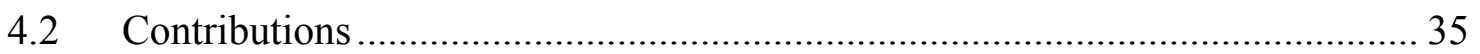

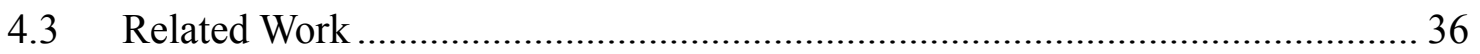

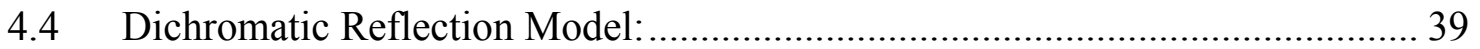

4.5 Inverse-Intensity Chromaticity (IIC) Space ……….......................................... 40

4.6 Surface Reflectance Decomposition ................................................................. 43

4.7 Specular Reflection Range Modeling............................................................. 47

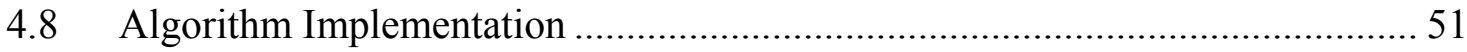

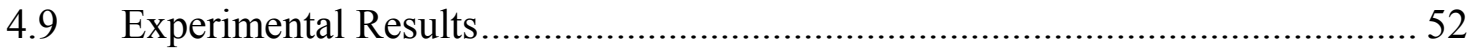


V. COLOR CONSTANCY BASED DICHROMATIC OBJECTS REFLECTION

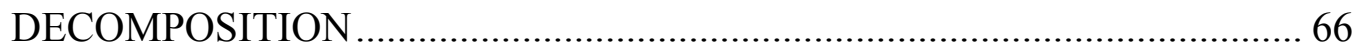

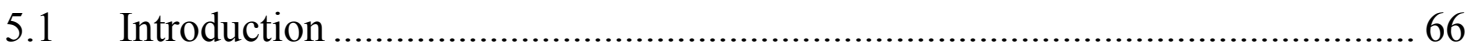

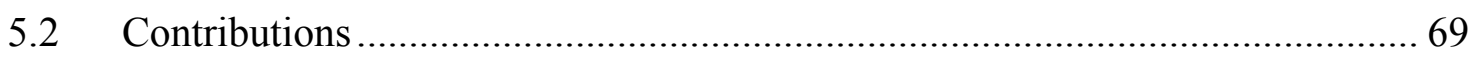

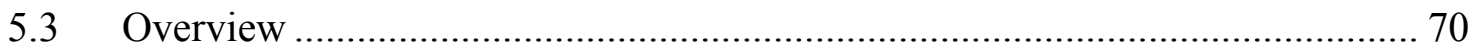

5.4 Dichromatic Reflectance Model.................................................................. 70

5.5 Diagonal Model Transformation ............................................................. 71

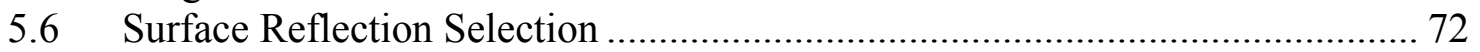

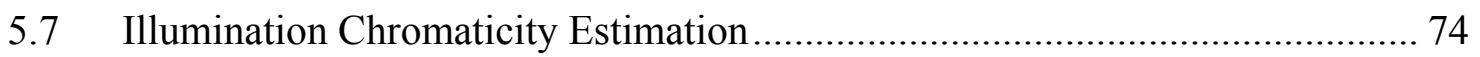

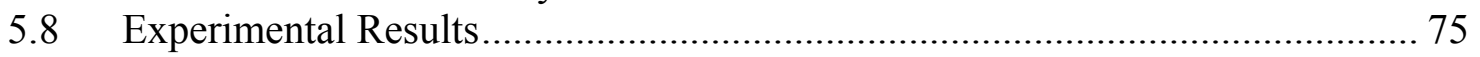

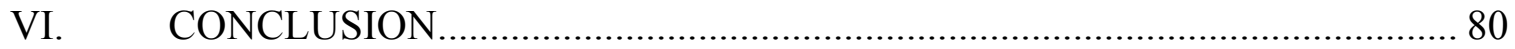

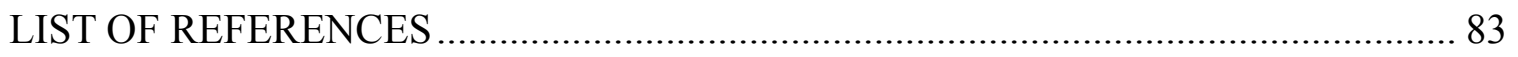

VITA 


\section{LIST OF FIGURES}

FIGURE

PAGE

Figure 3.1 Reflectance distribution separation over the IIC-space. (a) Input image. (b) Mapping image intensities of (a) over the IIC-space. (c) Separating the reflectance distribution into two distinct classes using the separation line (black) obtained from the proposed MSD method. (d) Classification of the projected image reflectance into diffuse reflectance distribution (red) and specular reflectance distribution (blue).

Figure 3.2 Specular reflectance shifting process: (a) Specular reflectance components (blue) shifting toward the new projected value (green) in the IICspace. (b) Specular reflectance new positioning in the diffuse reflection distribution.

Figure 3.3-A Reflection separation results: (a) Input image. (b) Separation result using our method. (c) Separation result using Tan et al. method [Tan , 2005].

Figure 3.4 Specular Removal process along the input image blue horizontal line. (a) Input image. (b) Input image after 2 iterations. (c) Input image after 4 iterations. (d) Cross-section of the image red channel for several iterations. (e) Cross-section of the image green channel for several iterations. (f) Crosssection of the image green channel for several iterations.

Figure 3.4 (Cont.) Specular Removal process along the input image blue horizontal line. (a) Input image. (b) Input image after 2 iterations. (c) Input image after 4 iterations. (d) Cross-section of the image red channel for several iterations. (e) Cross-section of the image green channel for several iterations. (f) Cross-section of the image green channel for several iterations.

Figure 4.1 Image (a) shows an outdoor scene image taken under mixed illumination. Images (b-d) portray the use of our proposed method to render scene illumination using different thresholds $(\eta R, \eta G, \eta B)$

Figure 4.2 An overview of the proposed reflectance separation method. (a) Synthetic input image. (b) Diffuse and specular points of the projected input image on the IIC-space. (c) A magnification of the portrayed projected image chromaticity values forming clusters of straight lines. (d) Resulted reflectance cluster separation using the proposed decomposition method. The blue line represents the separation line while the green and red clusters represent the specular and diffuse reflectance components respectively. 
Figure 4.3 (a) Transformation of the specular components (grey) coordinates by aligning the inverse-intensity axis of the IIC space with the reflection separation line (blue). (b) Specular components stretching process indicated by varying the threshold ratio $\eta>1$ (red) and $\eta<1$ (green)

Figure 4.5 Comparing the estimated power spectrum of eleven different illuminants with their relative real power spectra measured by a spectrometer.

Figure 4.6. Example of rendering scenes with uniform illumination distribution using our proposed method with the shown thresholds.

Figure 4.6. (Cont.) Example of rendering scenes with uniform illumination distribution using our proposed method with the shown thresholds.

Figure 4.7 Illustration of the presented decomposition method into specular components (green) and diffuse components (red) overlapped by the modeling process (blue). (a) Input image. (b) The result of rendering (a). (c) Reflectance separation and modeling over the red channel. (d) Reflectance separation and modeling over the green channel. (e) Reflectance separation and modeling over the blue channel.

Figure 4.8 Illustration of the presented decomposition method into specular components (green) and diffuse components (red) overlapped by the modeling process (blue). (a) Input image. (b) The result of rendering (a). (c) Reflectance separation and modeling over the red channel. (d) Reflectance separation and modeling over the green channel. (e) Reflectance separation and modeling over the blue channel.

Figure 4.9 Illustration of the presented decomposition method into specular components (green) and diffuse components (red) overlapped by the modeling process (blue). (a) Input image. (b) The result of rendering (a). (c) Reflectance separation and modeling over the red channel. (d) Reflectance separation and modeling over the green channel. (e) Reflectance separation and modeling over the blue channel.

Figure 5.1 The results of the proposed algorithm compared to other color constancy methods on the real-world dataset. The illumination angular error is shown under each image.

Figure 5.2 The results of the proposed algorithm compared to other color constancy methods on the real-world dataset. The illumination angular error is shown under each image.

Figure 5.3 The results of the proposed algorithm compared to other color constancy methods on the controlled indoor illumination dataset. The illumination angular error is shown under each image. 
Figure 5.4 The results of the proposed algorithm compared to other color constancy methods on the controlled indoor illumination dataset. The illumination angular error is shown under each image............................................. 79 


\section{CHAPTER I}

\section{Introduction}

\subsection{General Statement of the Problem Area}

In computer vision and image processing, the modeling of surface reflectance remains of vital importance for purposes involving surface analysis and image understanding. By separating objects' surface reflectance properties, powerful Lambertian-based methods can be effectively applied for object tracking, pattern classification, reconstruction and object recognition in real-world scenarios. They are also extremely useful for the more subtle tasks of shape from shading, binocular stereo vision, and motion vision. Scenarios that are considered by many algorithms often assume that only diffuse reflections are present, while specular ones are often considered to be negligible. However, specular reflections confuse many vision problems since they produce image attributes that do not bind directly with the intrinsic surface properties such as shape and spectral reflectance. Thus, methods that successfully separate both reflectance components diffuse and specular in images are highly desired for advancing current technology that suffers from the unyielding effects of this complex reflectance occurrence.

\subsection{Research Problem}

The main research problem of this dissertation is in determining a new mathematical framework in terms of both a domain of analysis as well as in the way real-world illumination could be determined through the RGB color model. A singular research problem that remains elusive and is addressed in this dissertation is in the development of 
an efficient separation method based on the Eigen-decomposition transformation plane in order to isolate the diffuse and specular reflectance information from color images. Secondary contributions in developing this new framework emanate from the facts that through this new transformation plane it now becomes possible to estimate real-world elimination as well as contend with the problem of color constancy.

\subsection{Signification of the Study}

The significance of this study relates directly to augmenting our understanding of the two key concepts in radiometry, illumination and reflectance. In so doing, the notion of color constancy is elucidated as well. These key concepts are intrinsically linked to advancing major research domains in computer vision and computer graphics. These include object recognition, motion vision, stereo vision, shape from shading and structure from motion, to name a few.

\subsection{Structure of the Research}

Chapter 2 provides the background on illumination and all the related factors that contribute to understanding the color model, particularly the RGB (Red-Green-Blue) model and how through such model one can characterize the specular and diffuse information and its power spectral density. The key concepts that relate to the research of this dissertation are summarized within the confines of radiometry and photometry as means to gauge an appreciation of electromagnetic radiation measurements, including visible light. This chapter also includes an overview of the illumination spectral power 
distribution, surface spectral reflectance, and essential camera properties within the context of this dissertation.

Chapter 3 addresses the separation of reflection components in scene images as means to improve the effectiveness of the aforementioned applications. Unlike many of the existing methods whose applications remain limited to the reflectance component, the proposed approach suggests the possibility of decomposing surfaces into two-distinct reflectance constituents solely based on color image information. The main contribution at this research stage is attributed to the development of an efficient separation method based on the Eigen-decomposition transformation plane, we call the mean shift decomposition, which is then used to isolate the diffuse and specular reflectance information from color images. The dissimilarities between the diffuse and specular reflectance distributions projected in this normalized color space become apparent and can be readily isolated. What is most important is developing appropriate rendering methods that benefit the scenes' illumination distributions while conserving their surface diffuse reflectance unaffected, leading to realistic illumination effects.

This novel process can be very useful in many applications that involve refining indoor and outdoor scenes environment appearance in the fields of computer graphics and computer vision. This proposed method is evaluated for its practical merit on several real-world illumination spectrums used to render scenes of both indoor and outdoor images. This method is also implemented on both synthetic and real-world images for visual appreciation as well as for gauging the different realistic rendering that take into account the concept of illumination. 
Chapter 4 introduces a novel scene illumination rendering technique based on the specular/diffuse separation concepts described in Chapter 3. Ancillary contributions of this chapter on the basis of the framework established in chapter 3 include: (1) the development of a complementary method to estimate the illumination reflectance component using the Mean-Shift Decomposition (MSD) technique that was described in Chapter 3; and (2) the establishment of a linear transformation method, similar to the methods involving changing the dynamic range of histograms as used in image enhancement techniques, in order to achieve effective illumination rendering. Implementation of this approach on synthetic and real-world scenes demonstrate that the proposed rendering method can produce real world illumination effects that can be appreciated under different thresholds that can be preset or manually changed by the user. The obtained "real" rendered results of this method are compared with many different indoor and outdoor real world illuminations spectral distributions for comparative purposes.

In chapter 5, the approach considered was derived more as a supplementary contribution by estimating illumination chromaticity in images based on the dichromatic reflectance approach introduced in Chapter 3. The aim was to prove that by projecting image reflectance into the inverse-intensity chromaticity space, image highlights can be selected which are then evaluated using histogram analysis to sift only those prominent surface reflectances that replicate the exact illumination chromaticity. The illumination chromaticity is estimated through the intersection of the first and second eigenvectors of the orthogonal basis of the principal component analysis. In so doing, the process no longer necessitates any type of preprocessing generally needed to isolate the reflectance 
highlight. The significant aims that have been attained in this chapter include: (1) a voting-scheme that assumes a small set of surface reflectance to be used as means to characterize the most prominent image pixels that best reflect the illumination chromaticity; and (2) the estimation of the illumination chromaticity accomplished by projecting the intersection of the first and second orthogonal bases onto the image chromaticity axis of the illumination chromaticity is IIC-space.

Chapter 6 concludes with a retrospective on the major contributions that are made through this dissertation and provides directions for future research. This includes a potential resolution to the automatic setting of thresholds as an alternative to the intersect of the first two eigenvectors in seeking a more accurate estimation of the real-world illumination; and the determining the distance separating the targeted new location of the specular pixel in the diffuse reflectance distribution and its current location. 


\section{CHAPTER II}

\section{Background}

\subsection{Introduction}

The natural ability of the human visual system to perceive the color of objects as being relatively constant under different illumination conditions, a phenomenon known as color constancy, is a remarkable processing feat that has attracted the attention of several researchers in the last decades. Visual systems matching this ability of perceiving natural scenes under varying illuminations still struggle in determining new paradigms that could emulate this phenomenon. It is this important issue that served as motivation to this dissertation work. In machine vision, the appearance of object surfaces is a crucial requirement for various applications in image rendering, illumination estimation, object recognition, etc. Objects, especially dielectric objects, have the property of exhibiting highlights in their surface, as will be seen in the next chapter, the objects' surface properties can be divided into two distinct reflectance components, diffuse reflectance and specular reflectance.

When an object is illuminated by a light source, based on its surface intrinsic properties, the light rays hit the object surface at some point. Some of the light ray bundles immediately reflect back into the air, while other rays penetrate the surface body. The penetrated light rays are called diffuse or body reflection, and the reflected light is called specular or interface reflection. Unlike diffuse reflections, specular reflections discerned from objects depend on illumination and viewing directions explaining their variant appearance. However, diffuse reflections depend only on illumination directions as 
indicated by their intensities. As a consequence, the first objective of this dissertation is to decompose objects reflectance components and then acquire diffuse only surface reflectance. This practice of recovering specular free object surfaces is believed to positively advance the operation of machine vision applications, which are often confused by the specular reflections. However, The capability of recovering the correct object surface color is limited to the knowledge of the actual illumination conditions in which the object scene was exposed. The illumination color changes can affect the appearance of the color descriptors governing the object surfaces. For objects exhibiting highlights, estimating the illumination color is important in order to accurately retrieve the image components representing the surface diffuse reflectance. These color constancy approaches and reflection-components separation are analyzed based on the physical appearance of object surfaces under real-world conditions. Therefore, it is important to consider understanding the physical interactions occurring between the illumination, surface reflectance, and device recordings such as digital cameras.

\subsection{Illumination Spectral Power Distribution}

The visible light spectrum is a portion of the electromagnetic radiation identified as a natural agent to stimulate human vision. Light is composed of a number of energy packets known as photons that travel in a straight direction and oscillate at a certain orientation at a spectrum of wavelength, and are visible within a range of $380 \mathrm{~nm}$ to 780 nm. All light sources emit several spectral power distributions containing all physical data describing the light color. These spectral distributions can be approximated using finite linear basis functions expressed as: 


$$
E(\lambda)=\sum_{i} e_{i} E_{i}(\lambda)
$$

where $E(\lambda)$ is the light spectral power distribution, $E_{i}(\lambda)$ is a basis function, and $e_{i}$ are the coefficients of the basis functions for $i \in\left\{1, \ldots, n_{b}\right\}$, where $n_{b}$ is the number of basis functions. In practice, three to five basis functions are sufficient to model most daylight spectra.

\subsection{Reflectance Basis functions}

The surface spectral reflectance describes the ability of an object to reflect illumination spectral distribution. It is defined as the ratio between the reflected spectral power distribution and the incident spectral power distribution. Similarly to illumination characteristics, reflectance can also be approximated using a finite set of linear basis functions. If the space of reflectance is decomposed into a finite set of $m_{b}$ basis functions $\boldsymbol{R}_{\boldsymbol{i}}$ with $i \in\left\{1, \ldots, m_{b}\right\}$, then the reflectance $R(\lambda)$ for a given wavelength $\lambda$ can be written as:

$$
R(\lambda)=\sum_{i} r_{i} R_{i}(\lambda)
$$

where $r_{i}$ are the coefficients of the basis functions. Generally, these basis functions are obtained by performing principle component analysis (PCA).

\subsection{Image Chromaticity}

Taking a set of tri-stimulus values $\{\mathrm{X}, \mathrm{Y}, \mathrm{Z}\}$ as the 3-D color space coordinate, the color of a point in the space is dependent on both the direction and magnitude of the vector. 
Any changes in the vector's magnitude will change the brightness of the color while changes in the vector's direction will modify the color's hue and saturation. The color chromaticity coordinates map tri-stimulus color channels into a two-dimensional space composed of hue and saturation color attributes. Therefore, colors are usually presented in a normalized form generally referred to as color chromaticity. The chromaticity components of the CIE color space are computed by normalizing each color value by the color sum:

$$
x=\frac{X}{X+Y+Z}, \quad y=\frac{Y}{X+Y+Z}, \quad z=\frac{Z}{X+Y+Z}
$$

Geometrically, the initiated normalization projects all colors onto the unit plane $X+Y+Z=1$. Therefore, the normalized coordinates $\{\mathrm{x}, \mathrm{y}, \mathrm{z}\}$ are independent of the length of the $\{\mathrm{X}, \mathrm{Y}, \mathrm{Z}\}$ vector, and similarly $x+y+z=1$. Even though the third coordinate $\mathrm{z}$ can be easily calculated given $z=1-x-y$, throughout this dissertation, we will refer to the image color chromaticity coordinates using the $\{x, y, z\}$ triplet form. 


\section{CHAPTER III}

\section{Surface Reflectance Components Separation from Single Color Images \\ Using the Mean-Shift Decomposition Technique}

\subsection{Introduction}

This chapter addresses the separation of reflection components in scene images as means to improve the effectiveness of the aforementioned applications. Unlike many of the existing methods that limit their application to the reflectance component, our approach considers analyzing the image intrinsic properties relating to both diffuse and specular reflectance components. We restrict our consideration to surfaces that are well represented by the dichromatic reflectance model introduced by Shafer [Shafer, 1985]. This model suggests the possibility of decomposing surfaces into two-distinct reflectance constituents solely based on color image information. Our approach studies the dissimilarities between the diffuse and specular reflectance distributions projected in a normalized color space in order to isolate information about the material properties in an image scene while preserving the surface geometry information.

We introduce a simple but efficient reflectance decomposition technique based in the Eigen-decomposition transform that we refer to as the Mean-Shift Decomposition (MSD) method. This technique provides a direct access to surface shape information through diffuse shading pixels seclusion. In addition, the proposed method does not require any local color segmentation process as it differentiates between both reflectance components through the proposed MSD method which differs from several proposed methods in the literature that operate by aggregating color information along each image plane. 


\subsection{Contributions}

The contributions involved in the proposed reflectance separation method reside in:

- Analyzing and separating the image reflectance component using the Mean-shift decomposition technique derived based on the Eigen-decomposition transform.

- Recovering the surface geometry information by isolating the image diffuse reflectance distribution.

- Formulating a specular reflectance removal process by shifting the specular reflectance components toward the decomposed diffuse reflectance distribution.

- Evaluating the proposed algorithm on several images comprising uniform color surfaces, multicolor surfaces, and highly textured surfaces.

\subsection{Overview}

Section 3.4 presents a literature review on different related methods used for accomplishing the diffuse/specular separation task. In section 3.5, a review is given on several types of reflectance models used for reflection measurements. This same section also describes the model as employed in the reflectance separation approach considered in this dissertation. Section 3.6 presents the chromaticity-color space used as a basis to the MSD technique. Sections 3.7 and 3.8 describe the classification of the image reflectance components using the $M S D$ method and the specular removal process, respectively. An outline of the proposed algorithm implementation is presented in section 3.9. Finally, we evaluate and summarize our proposed method in sections 3.10 and 3.11 . 


\subsection{Related Work}

Many methods have been proposed for separating reflection components using single or multiple input images of non-Lambertian objects (these are objects that deviate from the Lambert Law which assumes that luminance of a diffuse surface is the same in all directions; as formulated in 1760 by J. Lambert).

Based on color and image intensities, Sato and Ikeuchi [Sato, 1994] introduced a fourdimensional temporal-color space to analyze the diffuse and specular reflections. Their method has the ability to separate the reflection components using local interactions, however, it requires numerous input images with variation of illuminant directions. The benefit of using local analysis is that it admits highly textured scenes that do not contain piecewise constant diffuse colors. Lin et al. [Lin, 2002] used color histogram differencing to identify specular reflectance with multi-baseline stereo vision. Their method makes use of the image pixel color stability to the field of view changes, since colors of specular pixels are dependent on the scene viewing positions while the diffuse pixel colors are not. Lin and Shum [Lin, 2001] introduced a method that uses several sparse images registered under different illumination positions. Their method uses the neutral interface reflection model that combines finite dimensional basis functions [Parkkinem, 1989] and the dichromatic reflectance model [Shafer, 1985] to form a closed form linear surface reflectance.

Other methods based on multiple input images makes use of extrinsic cues such as polarizing filters to enable the recovery of a spatially-varying source colors [Nayar, 1996].

Wolff and Boult [Wolff, 1991] used a polarizing filter to separate both specular and diffuse reflection components from grey images. They noticed that diffuse reflections 
tend to be less polarized than the specular reflections along most incident reflection angles. On the other hand, reflectance separation methods that use a single input image have also attracted several considerations from the research community. Shafer [Shafer, 1985] introduced the dichromatic reflectance model suggesting the ability of decomposing an image into its specular and diffuse components based on the parallelogram distribution of colors in RGB space. Klinker et al. [Klinker, 1990] showed that the color histogram of the image diffuse reflection color forms a T-shaped distribution when the diffuse colors are the same along each point on an object's surface. The color distribution they proposed forms linear clusters representing the diffuse and specular pixels. By separating these clusters, the image becomes segmented into several regions of homogeneous reflectance color. Later, Tan et al. [Tan, 2005] iteratively compared the intensity logarithmic differentiation of an input image and its specular-free image. Their method reduced the specular component value of each pixel iteratively by considering one of its neighbor pixels as having a similar diffuse component characteristic. Mallick et al. [Mallick, 2006] applied a family of partial differential equations $(P D E)$ that iteratively erodes the specular reflectance component at each pixel.

\subsection{Reflection Model and Image Formation}

The use of physical reflectance measurements has shown to benefit the rendering of surface reflectance effectively. Such measurements can provide an ideal choice of parameters for existing reflectance models and can be used also to provide the basis for entirely other new reflectance models. For instance, the bidirectional reflectance distribution function $(B R D F)$ measurement describes the reflection of an opaque surface 
by measuring the ratio of the radiance $L$ reflected from the surface in a certain direction to the incident irradiance $I$ at a particular wavelength $\lambda$. We consider the $B R D F$ to be a five-dimensional function of wavelength and imaging geometry, whose measurement function $f$ can be described as:

$$
f(\Theta, \lambda)=\frac{d L\left(\theta_{r}, \phi_{r}\right)}{d I\left(\theta_{i}, \phi_{i}\right)}
$$

Where $\Theta=\left(\theta_{i}, \phi_{i}, \theta_{r}, \phi_{r}\right)$ defines the directions of the incident and reflected radiance in the local spherical coordinate system. A simple model that can be generated from the $B R D F$ measurement is the Lambertian reflectance model where the $B R D F$ is a constant function of the imaging geometry so that $f(\Theta, \lambda)=f(\lambda)$. Another common $B R D F$ model is the dichromatic model of reflectance derived by Shafer [Shafer, 1985] to represent dielectric materials. According to this model, the $B R D F$ of the surface can be decomposed into two additive components: (1) the specular reflectance representing the surface interface, and (2) the diffuse reflectance representing the surface body. This model assumes that each reflectance component can be expressed as being a function of wavelength and imaging geometry, which leads to the following expression of the dichromatic $B R D F$ model observed from a surface point $n$ :

$$
f(n, \lambda)=m_{d}(n) S_{d}(n, \lambda) E(n, \lambda)+m_{s}(n) S_{s}(n, \lambda) E(n, \lambda)
$$

where $m_{d}$ and $m_{s}$ are the geometrical scale factors for the diffuse and specular reflectance, respectively, which depend on the surface properties and illumination geometry $\Theta$. The $S_{d}$ and $S_{S}$ components are the diffuse spectral reflectance function and 
the specular spectral reflectance function, respectively, while $E$ is the spectral distribution function of the illuminant, and $n$ is the position of the surface point in a three-dimensional coordinate system.

Most methods that operate on dielectric inhomogeneous objects use the neutral interface reflection assumption (NIR) introduced by Lee et al. [Lee, 1990]. This assumption suggests that the spectral reflectance distribution of the specular reflection component $S_{S}$ is similar to the spectral energy distribution of the incident light $E$ [Finlayson, 2001] [Tominaga, 1989] [Lee, 1986]. As a result of this assumption Equation 3.2 can now be expressed as follows:

$$
f(n, \lambda)=m_{d}(n) S_{d}(n, \lambda) E(n, \lambda)+m_{s}(n) E(n, \lambda)
$$

By combining the dichromatic reflectance model represented by Equation 3.3 with the camera intrinsic properties, the image formation equation for a surface element illuminated by a light source is described as follows:

$$
\rho_{k}(n)=m_{d}(n) D_{k}(n)+m_{s}(n) S_{k}(n)
$$

where $D_{k}(n)=\int_{\Omega} S_{d}(n, \lambda) E(n, \lambda) C_{k}(\lambda) d \lambda$ and $S_{k}(n)=\int_{\Omega} E(n, \lambda) C_{k}(\lambda) d \lambda$

here $\rho_{k}=\left\{\rho_{r}, \rho_{g}, \rho_{b}\right\}$ is the RGB color vector response from a typical camera consisting of $k=\{r, g, b\}$ measurements, and $C_{k}=\left\{C_{r}, C_{g}, C_{b}\right\}$ is the sensor sensitivity of the three color channels over the range of the visible spectrum $\Omega . D_{k}$ and $S_{k}$ are the diffuse and specular image components respectively and are assumed to be unit length vectors $\left\|D_{k}\right\|=\left\|S_{k}\right\|=1$ 
Even though the dichromatic model was originally established to process materials such as glass, plastics, cloths, or even plant leaves, the model has also shown to be useful for applications involving human skin [Storring, 2000][Huynh, 2010][Tominaga, 2009].

Throughout this Chapter, we use the BRDF dichromatic model described in Equation3.4, and we assume that the illumination spectral power distribution is formed using a single and uniform illumination color $E(\lambda)$ independent from the image coordinates $n$. We note that the camera properties employed for image formation ignores the read noise and gain factor.

\subsection{Image-Chromaticity Color Space}

Color space transformations have shown to help exploring the knowledge of the illuminant attributes in color images, which results in describing the image diffuse information independently [Park, 2007][Zickler, 2008][Tan, 2004]. By shifting or projecting image intensities into a specified color space, depending on the motivated application, one can linearly combine the three image color channels to obtain either one or two distinct diffuse channels. Zickler et al. [Zickler, 2008] proposed a color space referred to as $S U V$ color space that isolates two-predominantly diffuse channels while retaining the entire specular components information apart. Similarly, Park [Park, 2003] isolated the diffuse reflectance channels while maintaining a similarity to the HSI color space using a linear transformation described by a matrix $L$ and a rotational transformation represented by a matrix $R$. The two matrices are chosen such that the third image color axis is aligned with the illumination color, which results in revealing a 
channel highly insensitive to specular reflections while leaving the other two remaining channels consisting mostly of diffuse components. On the other hand, Tan et al. [Tan, 2005] obtained a specular free image channel using a source dependent non-linear transformation of the RGB space. This transformation yields a positive monochromatic image that depends directly on diffuse shading information. Similar to Tan and colleagues' method, Yoon and Kweon [Yoon, 2006] proposed another non-linear transformation strictly relied on white illumination that produces also a positive grayscale image dominated by diffuse reflectance components and independent from specular effects.

In this dissertation we consider a different approach from the color transformation methods discussed. It is important to analyze the reflectance components distribution in the RGB color space to efficiently describe the correlation between those two reflection constituents. As mentioned in the previous section, assuming a dichromatic model object, the spectral reflectance distribution of the specular reflection component is similar to the spectral energy distribution of the incident light. Tan et al. [Tan, 2004b] introduced a new space transformation to analyze the relationship between illumination color and image intensity identified as inverse-intensity chromaticity space (IIC). Initiated mainly for illumination color estimation, we base our method on the IIC-space to identify the image pixels representing the diffuse and specular reflections. By projecting an RGB image into the IIC-space, the distribution of the image reflectance forms a set of straight lines expressed by the following equation:

$$
\sigma_{k}(n)=r \frac{1}{\sum_{j \in k} \rho_{j}(n)}+\omega_{s, k}(n)
$$


where $r=m_{d}(n)\left(\omega_{d, k}(n)-\omega_{s, k}(n)\right)$.

Here, $\sigma_{k}$ is the image intensity chromaticity of $\rho_{k} ; \omega_{d, k}$ and $\omega_{s, k}$ are the diffuse and specular reflectance chromaticity of $D_{k}$ and $S_{k}$ respectively. Figure 3.1.b-c show the distribution of the image green-channel projected on the IIC-space. These straight lines portraying both reflection components consist of several sets of different $m_{d}$ values representing the geometrical surface properties of the diffuse reflectance. These values depict the orientation of the reflection lines since they are associated with the gradient $r$ in Equation 3.5.

It can be observed empirically that as the object surface texture measurement is small (i.e. smooth surface), the diffuse reflectance distribution characterized in the IIC-space is relatively dense and is prone to merge with the specular reflectance components. However, if the object surface texture measurement is large (i.e. rough surface), the diffuse reflectance distribution becomes instead relatively sparse. Therefore, by understanding the distribution of the image reflectance properties in the IIC-space, the specular/diffuse separation process can be accomplished by finding the maximum reflectance disparities between the image pixels representing the specular and diffuse reflections.

In section 3.7, we describe how these reflection line differences can be categorized to separate the image diffuse and specular components using a simple method, based on the Eigen-decomposition transformation, which we call Mean-Shift Decomposition (MSD).

\subsection{Image Reflectance Components Classification}

The ability to determine the specular highlight regions has always been an ill-posed 
problem for several specular/diffuse separation methods. Previously, some methods require explicit color segmentation techniques to handle specular regions in multicolored surfaces especially when dealing with objects having highly textured surfaces and when processing single input images. In comparison to those methods, our proposed technique finds the maximum variance of image reflectance pixels to isolate both reflectance component distributions distinctly. This section introduces the Mean-shift decomposition method, a simple technique for specular and diffuse reflectance distribution classification over the IIC-space based on the Eigen-decomposition transform. Here we consider a twoclass problem where the classes are labeled to describe a diffuse reflectance class or a specular reflectance class.

Consider a vector $\boldsymbol{x}_{i}: \mathbb{R}^{2} \rightarrow[0,1]$ where $i=1,2, \ldots, N$, representing a sample point of the image reflectance components projected on the IIC-space and a matrix $\boldsymbol{X}$ denoting a set of all $N$ points $\boldsymbol{X}=\left[x_{1}, x_{2}, \ldots, x_{N}\right]$. Our objective is to find a separation line that maximizes the variance of the projected reflectance data. Let $\boldsymbol{U}$ denotes the direction of this line defining a new orthonormal basis of the projected reflectance values. These points can be expressed such that:

$$
Y=U^{T} X
$$

where $\boldsymbol{Y}=\left[y_{1}, y_{2}, \ldots, y_{N}\right]$ denotes the coordinates of the reflection points in the new basis, here we choose $\boldsymbol{U}^{T} \boldsymbol{U}=1$ as a constrain to maximize the reflectance variance. The mean of the projected reflectance components into the new basis line is $\boldsymbol{U}^{T} \overline{\boldsymbol{X}}$ where $\overline{\boldsymbol{X}}$ is the sample set mean given by: 


$$
\bar{X}=\frac{1}{N} \sum_{i=1}^{N} X_{i}
$$

and the variance of the image reflectance components is given by:

$$
\operatorname{Var}(X)=\frac{1}{N} \sum_{i=1}^{N}\left\{U^{T} X_{i}-U^{T} \bar{X}\right\}^{2}=U^{T} C U
$$

where $C$ is the image reflection components covariance matrix defined as:

$$
C=\frac{1}{N} \sum_{i=1}^{N}\left(X_{i}-\bar{X}\right)\left(X_{i}-\bar{X}\right)^{T}
$$

By Maximizing the projected variance in Equation 3.8 with respect to $\boldsymbol{U}$, the new orthogonal basis is represented by the eigenvectors of the covariance matrix $\boldsymbol{C}$ such that:

$$
C U=\Lambda U
$$

Where $\Lambda$ is a diagonal matrix of decreasing eigenvalues $\lambda$ and $\boldsymbol{U}$ is an orthonormal matrix of corresponding eigenvectors. The maximum variance separating the image diffuse and specular reflectance is obtained when the vector $\boldsymbol{U}$ corresponds to the highest eigenvalue. It is clear that the line separating the reflectance components into two distinct classes of diffuse and specular reflections intersects both reflection distributions, and is oriented according to the direction variations of the straight lines obtained by projecting the image into the IIC-space.

The MSD method finds the correlated features associated with each reflectance components mainly promoted in the center of the projected image cluster. This 
correlation occurring between both reflectance distributions rises for the fact that the image reflectance components projected on the IIC-space are characterized according to the image intensities distribution. Therefore, shifting the separation line represented by the direction of the new basis axis $\boldsymbol{U}$ to the center of the reflection distribution approximates the location that considerably relates both reflectance components in the IIC-space. The separation line is consequently shifted toward the center of the distance separating the diffuse and specular reflectance distributions means. We define the expression of the $M S D$ separation line as:

$$
f_{k}(n)=\tilde{u}\left(\frac{1}{\sum_{j \in k} \rho_{j}(n)}-\bar{x}_{1, k}\right)+\bar{x}_{2, k}
$$

where the gradient $\tilde{u}$ denotes the direction derived from the eigenvector of the orthonormal basis $\boldsymbol{U}$ corresponding to the highest eigenvalue in $\Lambda . \bar{x}_{1, k}$ and $\bar{x}_{2, k}$ are the mean of the projected image reflectance on the IIC-space corresponding to the matrix $X$. The proposed separation line in Equation 3.11 can efficiently partition the reflection distribution into two separate reflection classes as shown in Figure 3.1.d. The specular reflection components represented in the image can now be easily located by mapping the mean value of the brightest specular blob into the IIC-space. 


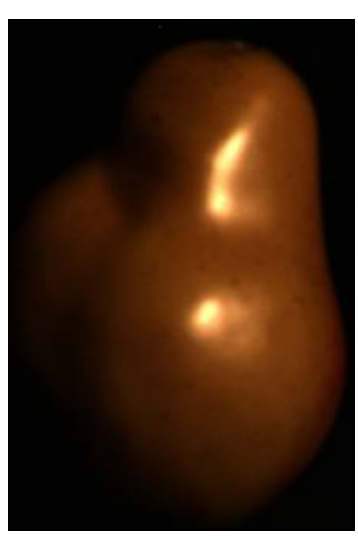

(a)

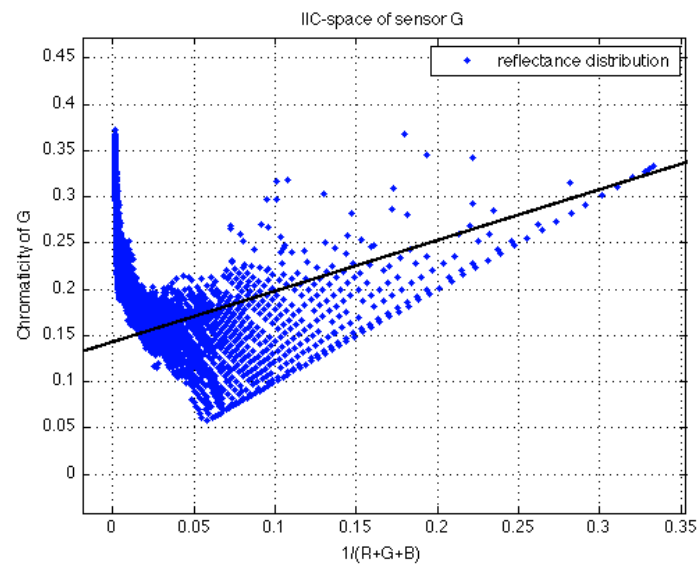

(c)

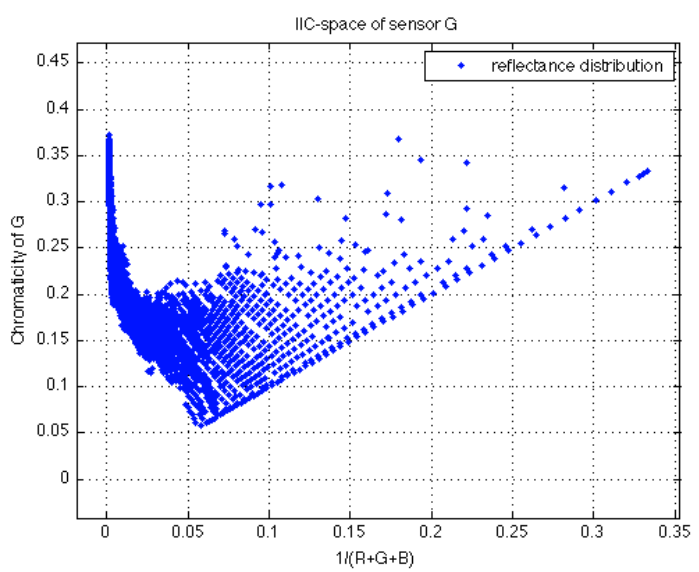

(b)

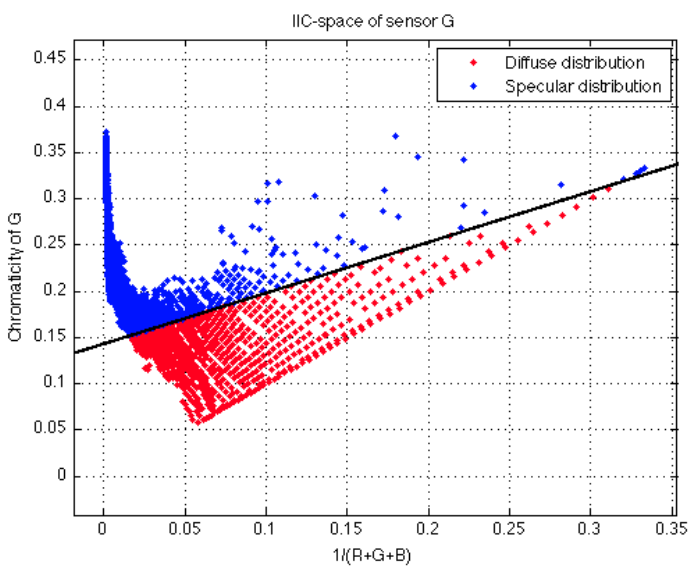

(d)

Figure 3.1 Reflectance distribution separation over the IIC-space. (a) Input image. (b) Mapping image intensities of (a) over the IIC-space. (c) Separating the reflectance distribution into two distinct classes using the separation line (black) obtained from the proposed MSD method. (d) Classification of the projected image reflectance into diffuse reflectance distribution (red) and specular reflectance distribution (blue).

\subsection{Specular Reflection Removal}

The dichromatic reflectance model suggests the possibility of decomposing an image into its specular and diffuse components based on image color information. After locating the image pixels representing the image diffuse and specular reflectance using the approach proposed in the previous section, in this section, we recover the diffuse properties of the 
specular distribution components by shifting the specular reflectance distribution components within the diffuse reflectance distribution in the IIC-space. This process has the advantage of preserving the surface color information and the diffuse reflection properties encoded in the geometric scale factor $m_{d}$.

Finding the shifting distance between each specular element and its new diffuse value is an important process for robust diffuse reflectance estimation. Let $C_{i, k}$ where $i=1,2, \ldots$, $N$, denotes the current specular reflection value projected on the IIC-space. And let $N_{i, k}$ denotes the newly shifted $C_{i, k}$ into the diffuse reflectance distribution by a Euclidean distance $D_{i}$ expressed as:

$$
D_{i}=\left(d_{i, x}^{2}+d_{i, y}^{2}\right)^{1 / 2}
$$

where $d_{i, x}$ and $d_{i, y}$ are the shifting distances over the inverse-intensity axis and image chromaticity respectively, Figure 3.2.a. We derive $d_{i, x}$ as follows:

$$
\begin{aligned}
& d_{i, x}=\frac{1}{\sum_{j \in k} N_{i, j}}-\frac{1}{\sum_{j \in k} C_{i, j}} \\
& d_{i, x}=\frac{1}{\sum_{j \in k} C_{i, j}-\varepsilon}-\frac{1}{\sum_{j \in k} C_{i, j}} \\
& d_{i, x}=\frac{\varepsilon}{\sum_{j \in k} C_{i, j}\left(\sum_{j \in k} C_{i, j}-\varepsilon\right)}
\end{aligned}
$$

and $d_{i, y}$ as follows: 


$$
\begin{gathered}
d_{i, y}=\frac{C_{i, k}}{\sum_{j \in k} C_{i, j}}-\frac{N_{i, k}}{\sum_{j \in k} N_{i, j}} \\
d_{i, y}=\frac{C_{i, k}}{\sum_{j \in k} C_{i, j}}-\frac{C_{i, k}-\varepsilon}{\sum_{j \in k} C_{i, j}-\varepsilon} \\
d_{i, y}=\frac{\mathcal{E}\left(\sum_{j \in k} C_{i, j}-C_{i, k}\right)}{\sum_{j \in k} C_{i, j}\left(\sum_{j \in k} C_{i, j}-\varepsilon\right)}
\end{gathered}
$$

where the threshold $\varepsilon$ defines the minimum value measured of $C_{i, k}$ and the difference of the maximum diffuse reflectance value of $D_{i, k}$ with respect to the minimum specular reflectance value of $S_{i, k}$, expressed as follows:

$$
\mathcal{E}=\left(C_{i, k}\right)_{\min }+\left(S_{i, k}\right)_{\min }-\left(D_{i, k}\right)_{\text {max }}
$$

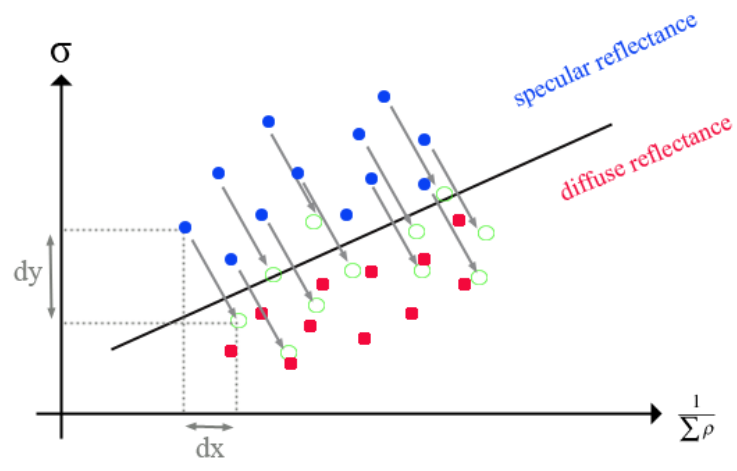

(a)

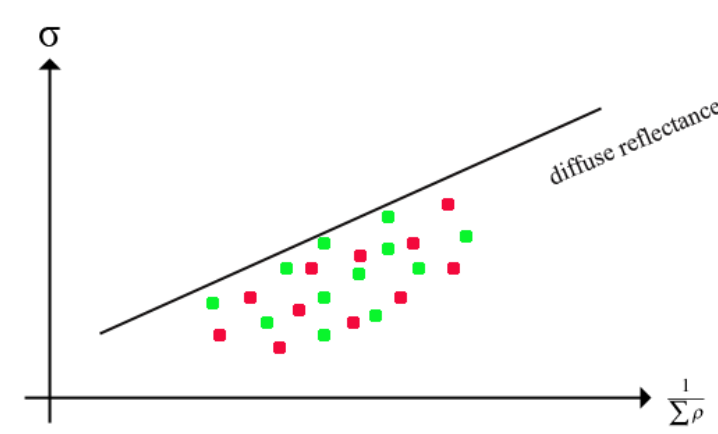

(b)

Figure 3.2 Specular reflectance shifting process: (a) Specular reflectance components (blue) shifting toward the new projected value (green) in the IIC-space. (b) Specular reflectance new positioning in the diffuse reflection distribution. 


\subsection{Algorithm Implementation}

Algorithm 3.1 outlines the steps and general data flow of the specular reflectance decomposition algorithm described in this chapter. The algorithm proceeds as follows: during the first stage, the input image intensities are mapped to the IIC-space by calculating the inverse-intensity sum and the image chromaticity for every channel $k$ (line1-3). The next step is to find a separation line that classifies each image intensity components into diffuse reflectance or specular reflectance. The means of the IIC-space coordinates representing the inverse image intensities and the image chromaticities are determined in order to find the image covariance matrix in Equation 3.9 (line 4-5) and to shift the separation line toward the center of the reflectance distribution (line 8).

The final step to obtain the separation line is to calculate the gradient of the line given by Equation 3.11. After sorting the eigenvalues (line 7), the eigenvector direction of the highest eigenvalue is used as the gradient of the line separation equation. The next stage consists on finding the distance separating the targeted new location of the specular pixel in the diffuse reflectance distribution and its current location. The distance along the inverse-intensity axis (line 14) and the image chromaticity (line 15) are calculated for every specular reflectance distribution constituent. The Euclidian distance in Equation 3.12 is then obtained before the specular value is shifted (line 16-17). Finally the three processed RGB channels are combined to produce a specular-free image (line 23). 


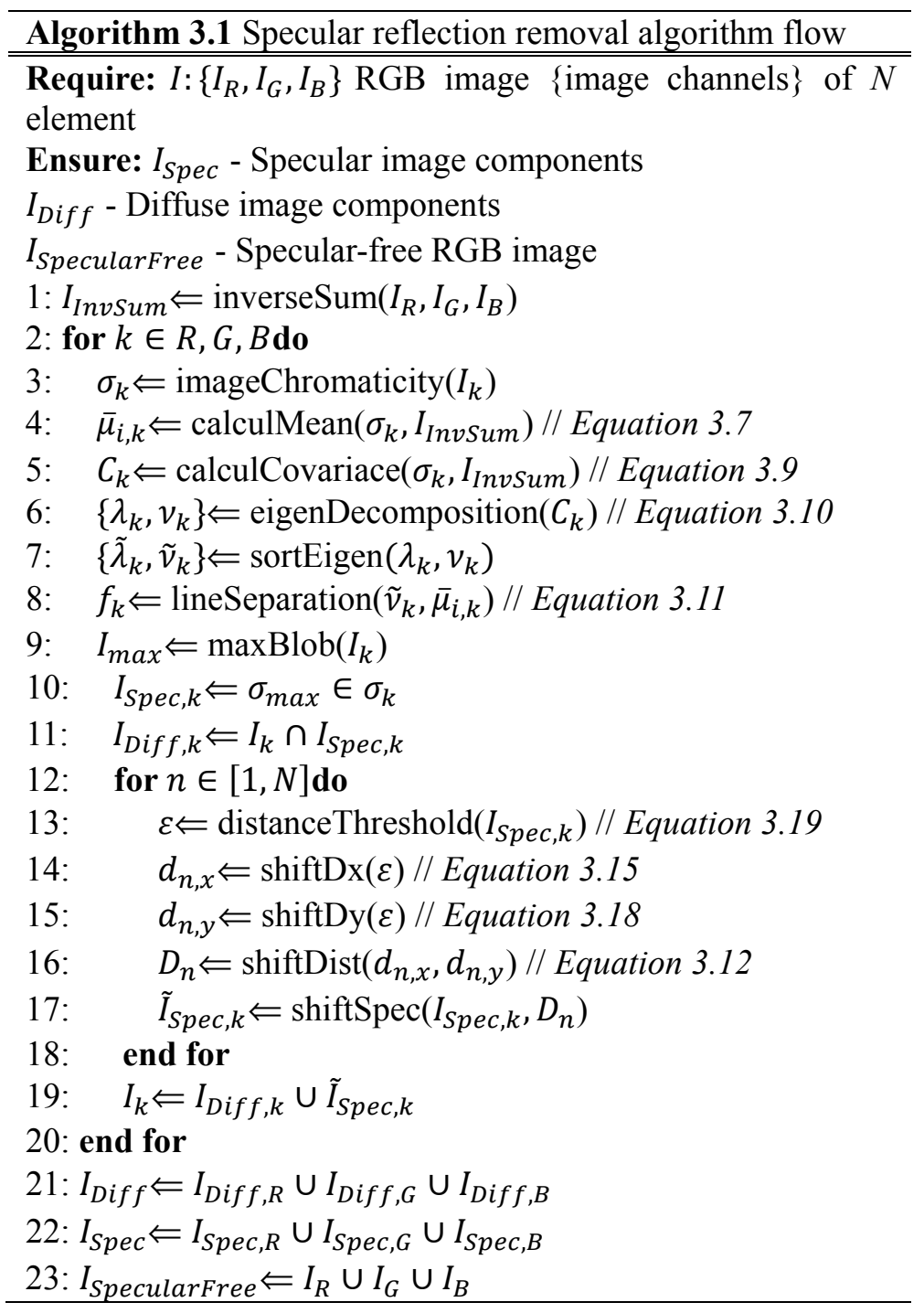

\subsection{Experimental Results and Discussions}

The proposed method was evaluated using several real and synthetic images from the color image dataset used in [Tan, 2005]. Different image surfaces were targeted in our experiment especially images consisting of uniform colored surfaces, multicolored surfaces, and highly textured surfaces. The images were registered using a progressive 3CCD digital camera SONY DXC-9000, by setting the camera correction option off. Also 
to ensure that the output of the camera is linear to the flux of incident light, a Photo Research PR-650 spectrometer was used.

Figure 3.3-B shows the results of recovering the diffuse image reflectance from a uniform surface color image. The obtained result is achieved using only two iterations and correctly handles all reflectance regions altered by the specular reflections. Also, since the proposed method processes each channel independently, all image color channels recovered the surface shape efficiently. The images in this case were processed without making use of the illuminant color and by setting the calculated Euclidian distance $D$ in Equation 3.12 to a constant value along all reflectance distribution components.

It is important to note that this practice of keeping $D$ constant does well only on images with uniform color surfaces. However, for multicolored images, this distance has to be calculated independently for every specular reflectance component in order to achieve acceptable results. In Figure 3.4, cross-sections of all input image three-color channels portrayed in Figure 3.3 are shown to describe the iteration process performance.

The solid blue horizontal line in Figure 3.4.a represents the cross-section area used to portray the iteration results in Figure 3.4.d-f. In each iteration process, it is noticeable that the surface color tone is preserved and attempts to complete the signal shape of the diffuse reflectance components. On the third and fourth iteration, we notice the specular signal flattening on a nearly constant pixel value resulting in wrong reflectance component separation. Another experiment is performed on multicolored and significantly textured images as shown in Figure 3.5. Looking closely at the pear in Figure 3.5.a-c, we notice that the diffuse texture barely visible in the input image is exposed when the specular effects are removed. Similarly in Figure 3.5.d-f, the fish 
texture is recovered while preserving the surface color of the object. Figure 3.5.g-i shows the results of processing several object surfaces grouped in one single image. Some of the surfaces are effectively recovered such as the fish object and the dole with blue scarf, however, other objects such as the yellow ball and some of the monster figurine surface were still suffering from the specular reflection effects.

Finally, we compare our algorithm with one of the well-known specular/diffuse reflection separation methods in the literature proposed by Tan et al. [Tan, 2005] in Figure 3.3-A. The figure compares both methods' results applied to a 3D head object with a uniform surface color. Both the shape surface color and the surface shade properties were efficiently estimated using our proposed method, similarly to Tan's et al. method where the surface shape was effectively reconstructed and the specular reflections were perfectly removed, however, the surface color was estimated inaccurately.

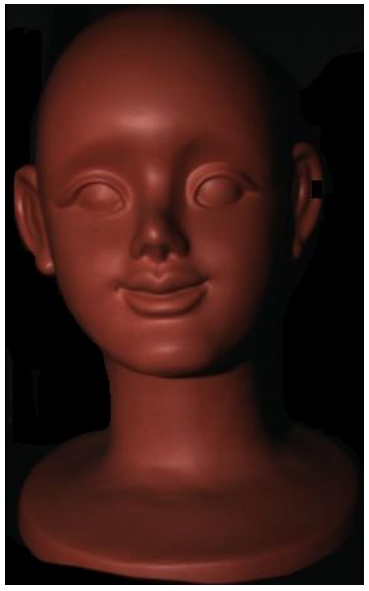

(a)

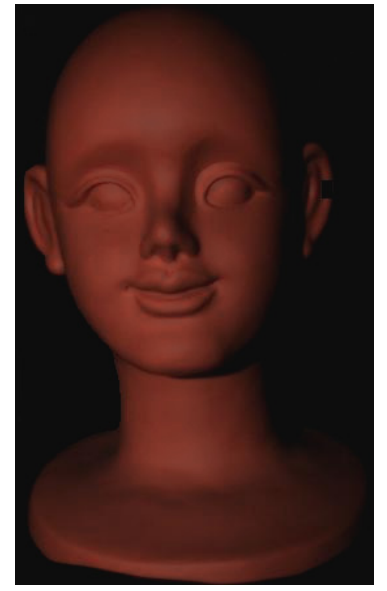

(b)

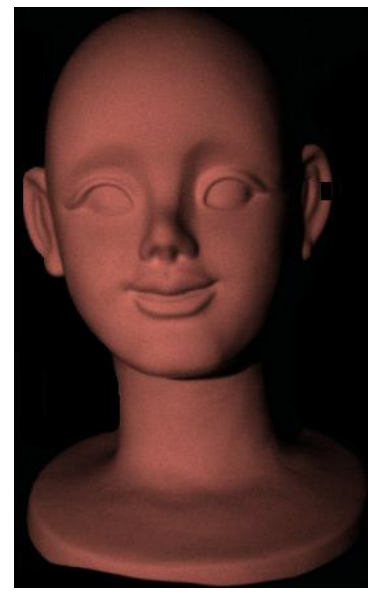

(c)

Figure 3.3-A Reflection separation results: (a) Input image. (b) Separation result using our method. (c) Separation result using Tan et al. method [Tan , 2005]. 


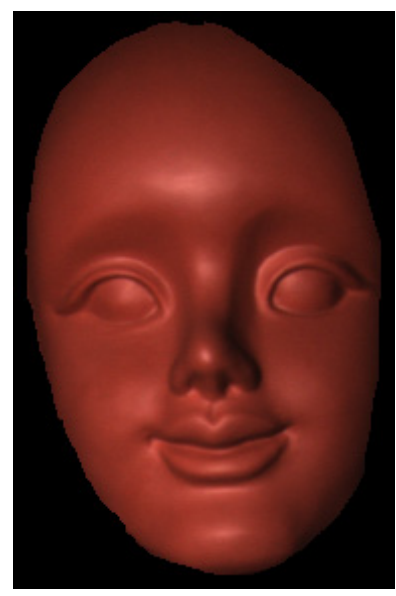

(a) Input image

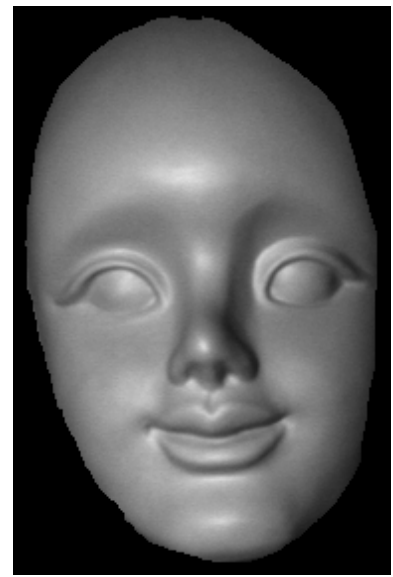

(d) Input Red Channel

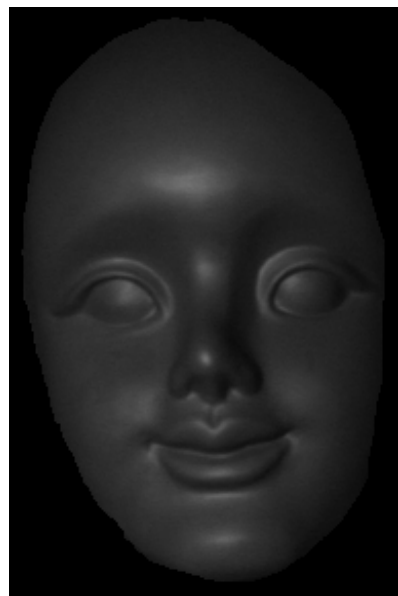

(g) Input Green Channel

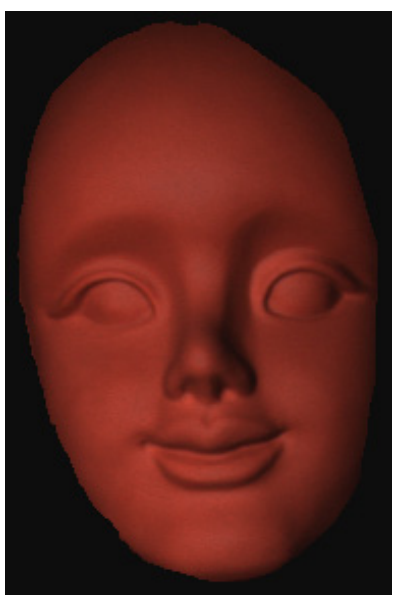

(b) Diffuse Reflection

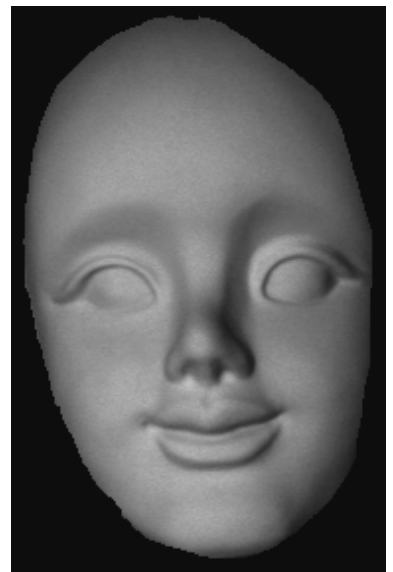

(e) Red Channel Diffuse Reflection

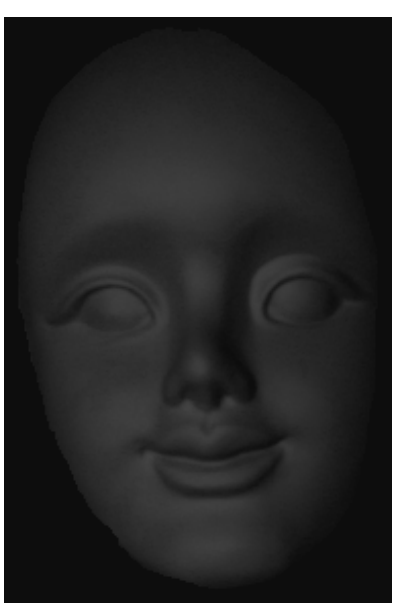

(h) Green Channel Diffuse Reflection

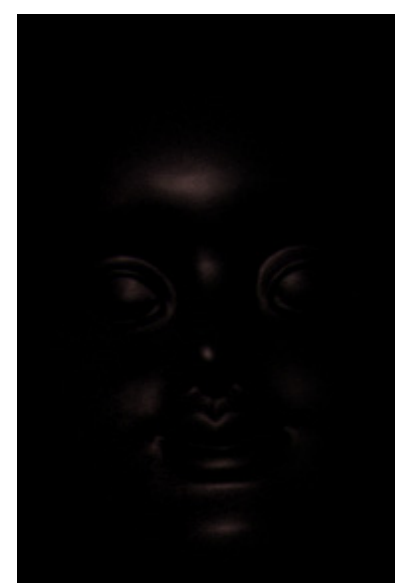

(c) Specular Reflection

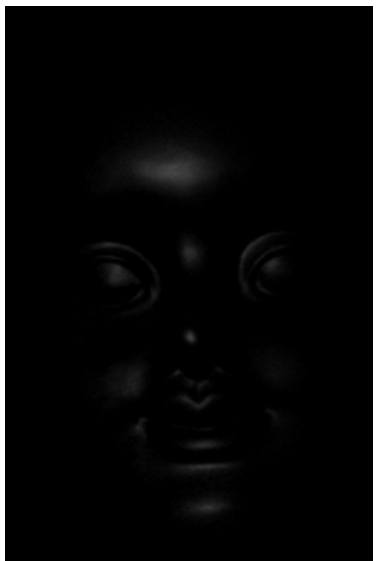

(f) Red Channel Specular Reflection

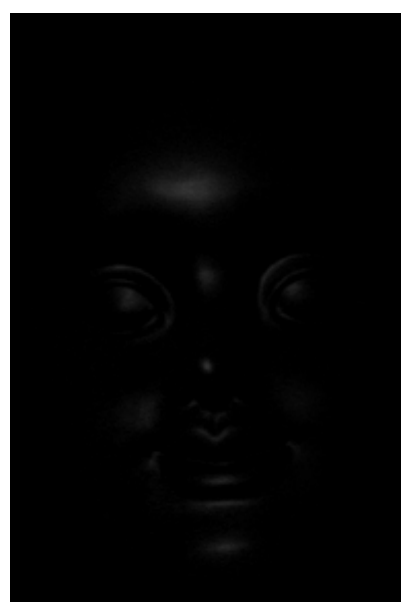

(i) Green Channel Specular Reflection

Figure 3.3-B Reflection separation results "Red face front view" having a uniform color surface along RGB color channels. 


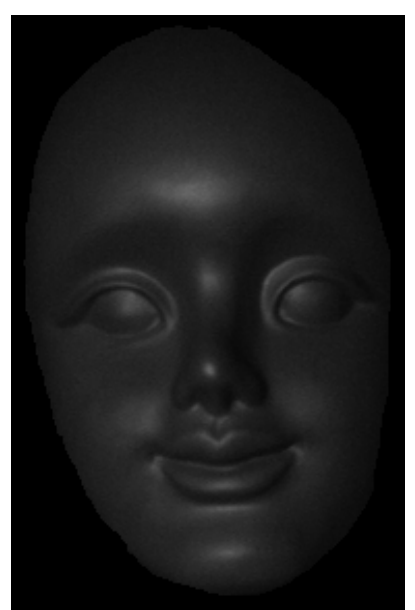

(j) Input Blue Channel

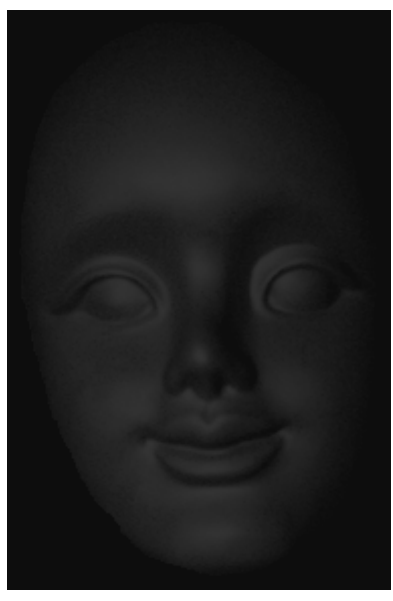

(k) Blue Channel Diffuse Reflection

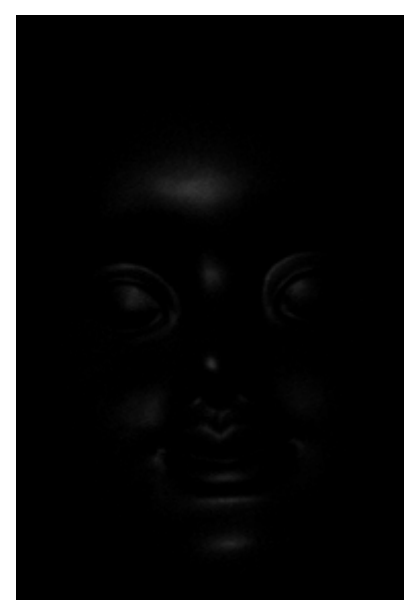

(1) Blue Channel Specular Reflection

Figure 3.3-B (Cont.) Reflection separation results "Red face front view" having a uniform color surface along RGB color channels.

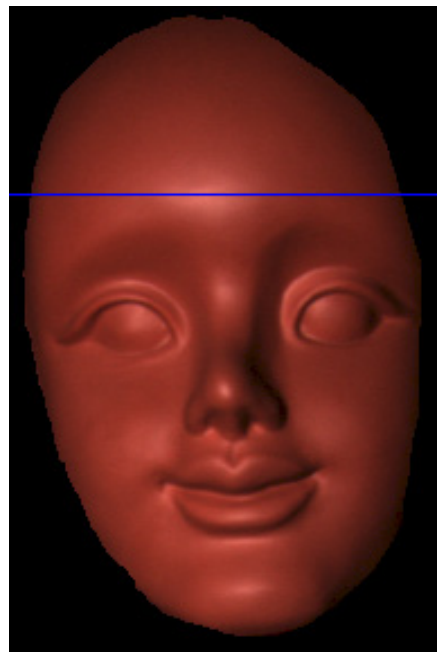

(a)

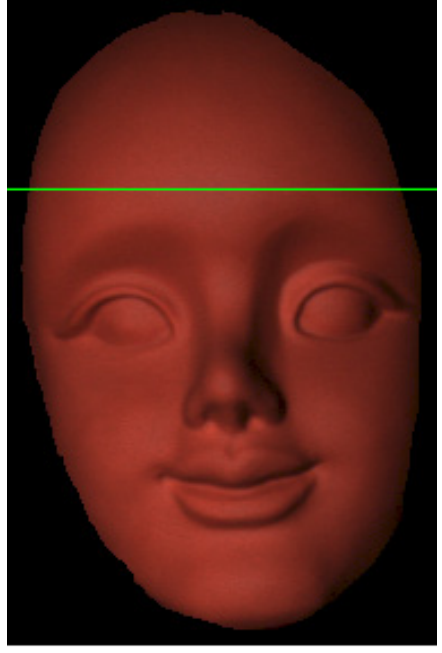

(b)

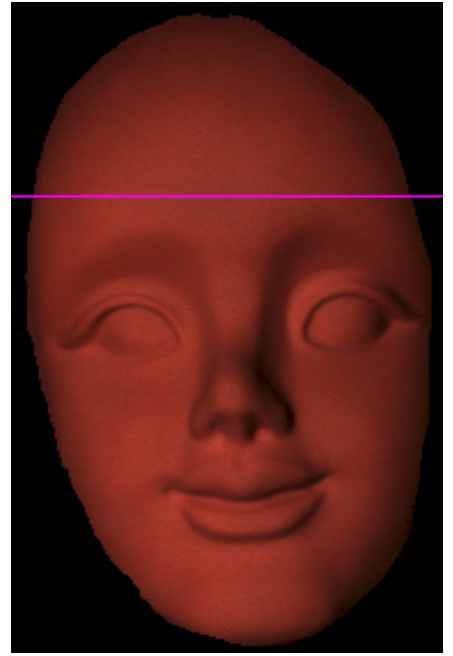

(c)

Figure 3.4 Specular Removal process along the input image blue horizontal line. (a) Input image. (b) Input image after 2 iterations. (c) Input image after 4 iterations. (d) Cross-section of the image red channel for several iterations. (e) Cross-section of the image green channel for several iterations. (f) Cross-section of the image green channel for several iterations. 


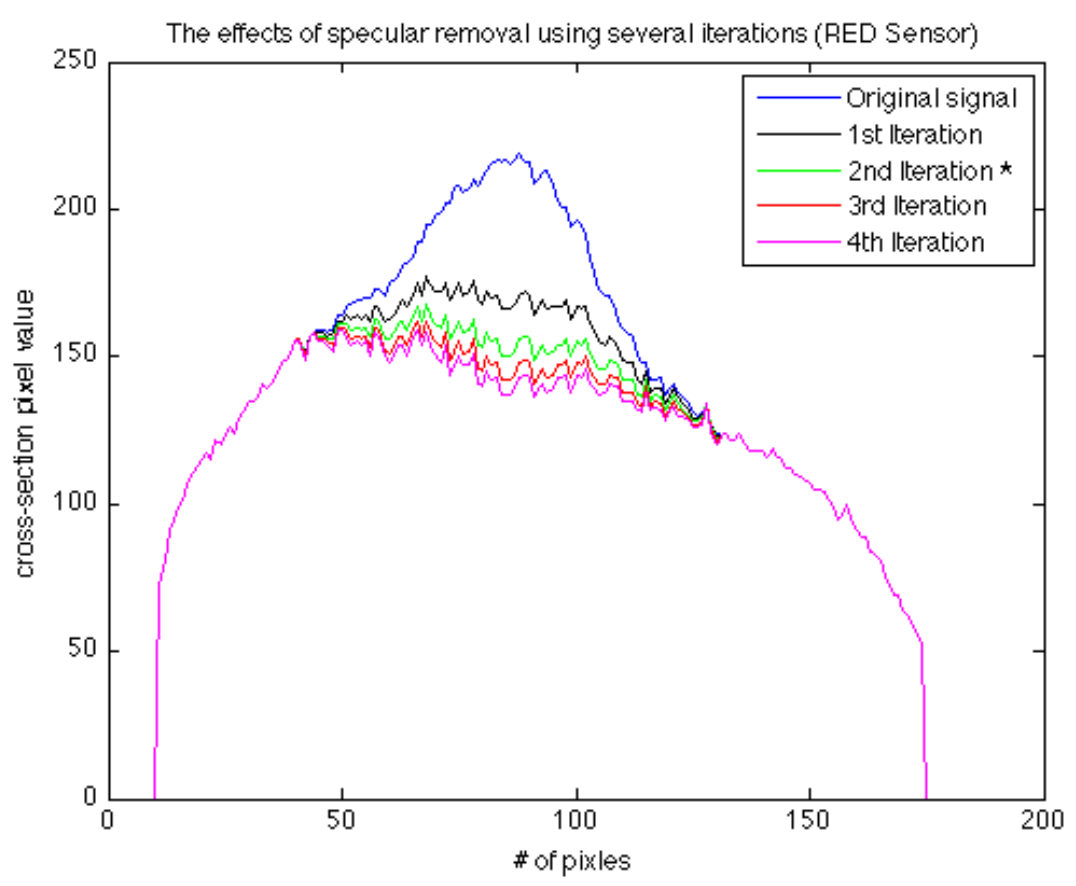

(d)

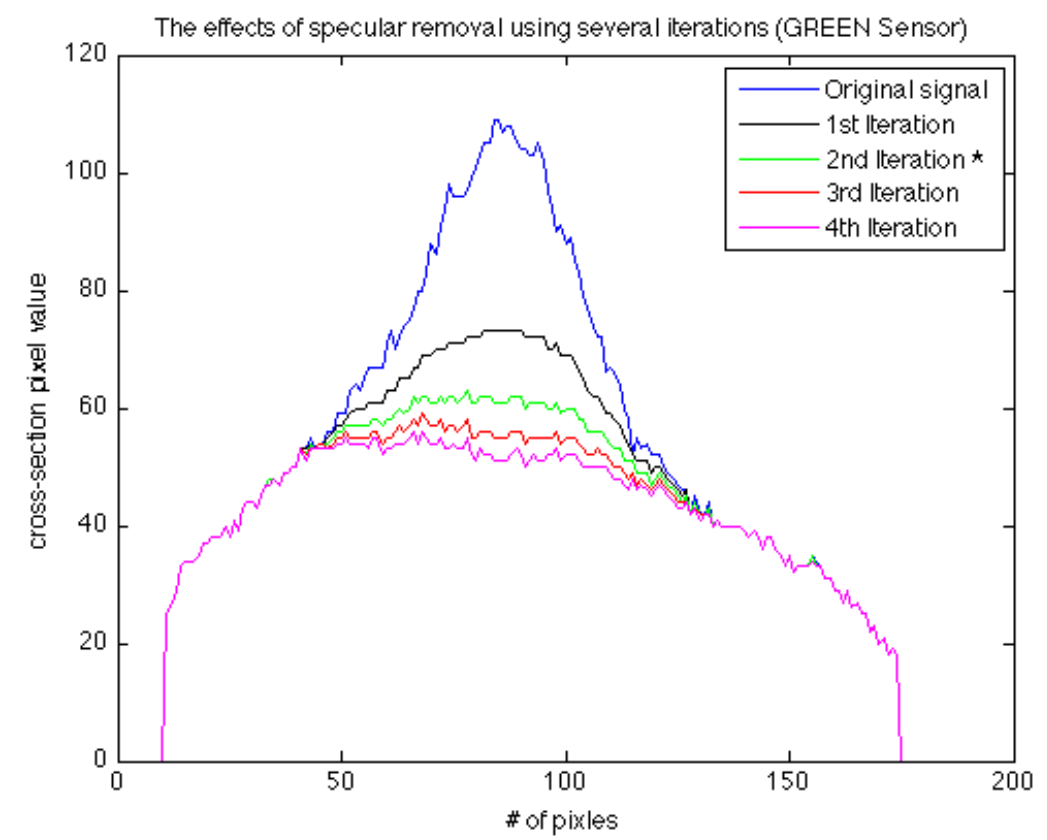

(e)

Figure 3.4 (Cont.) Specular Removal process along the input image blue horizontal line. (a) Input image. (b) Input image after 2 iterations. (c) Input image after 4 iterations. (d) Cross-section of the image red channel for several iterations. (e) Cross-section of the image green channel for several iterations. (f) Cross-section of the image green channel for several iterations. 


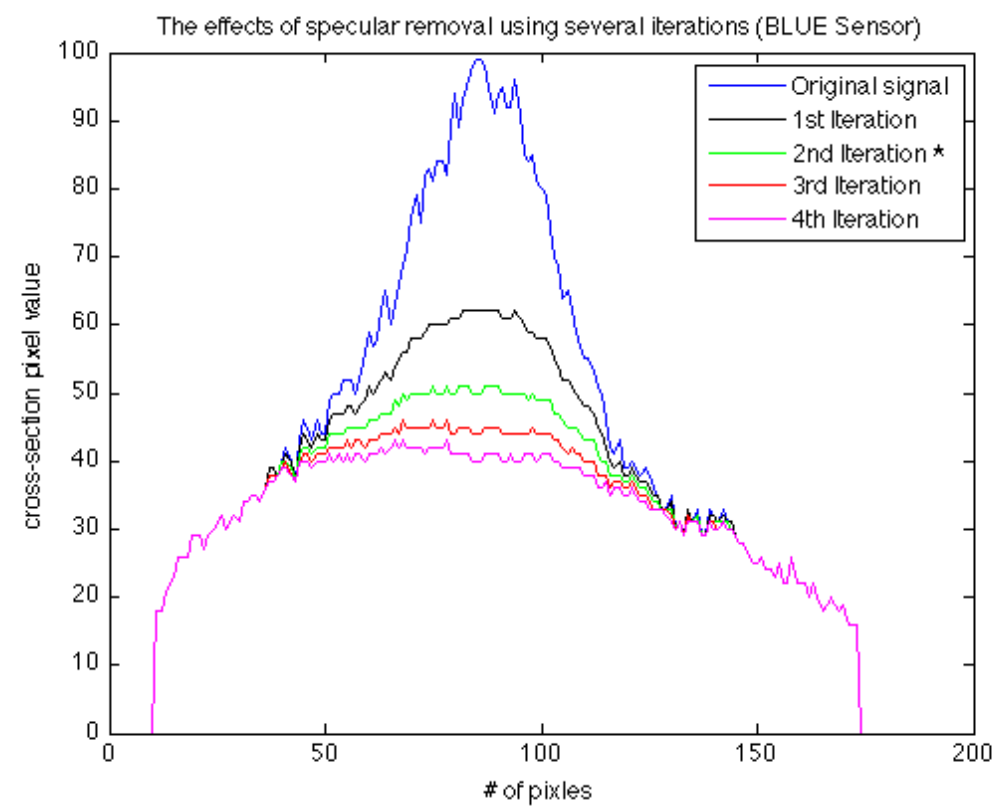

(f)

Figure 3.4 (Cont.) Specular Removal process along the input image blue horizontal line. (a) Input image. (b) Input image after 2 iterations. (c) Input image after 4 iterations. (d) Cross-section of the image red channel for several iterations. (e) Cross-section of the image green channel for several iterations. (f) Cross-section of the image green channel for several iterations.

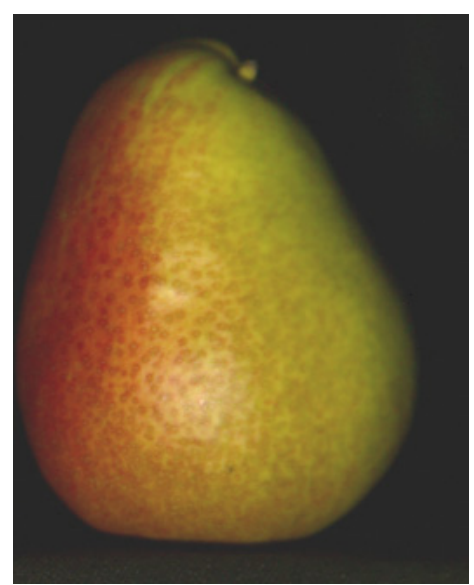

(a) Input Image

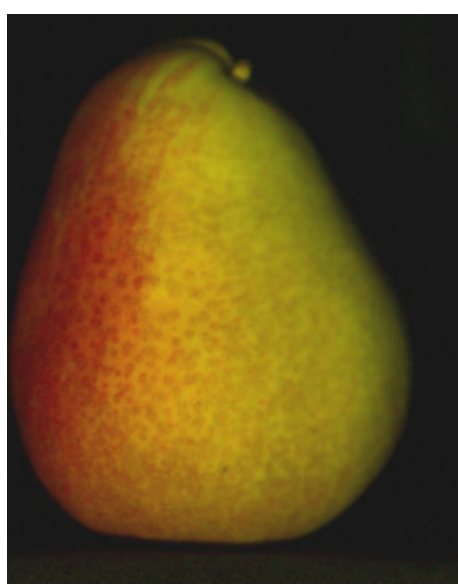

(b) Diffuse Reflection of (a)

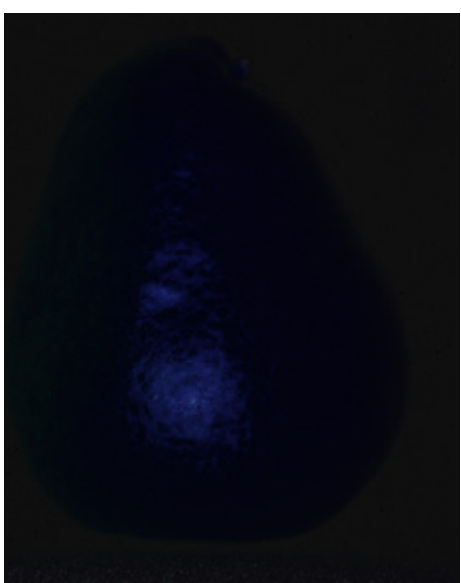

(c) Specular Reflection of (a)

Figure 3.5 Reflection separation results for input images having multicolor surfaces. 


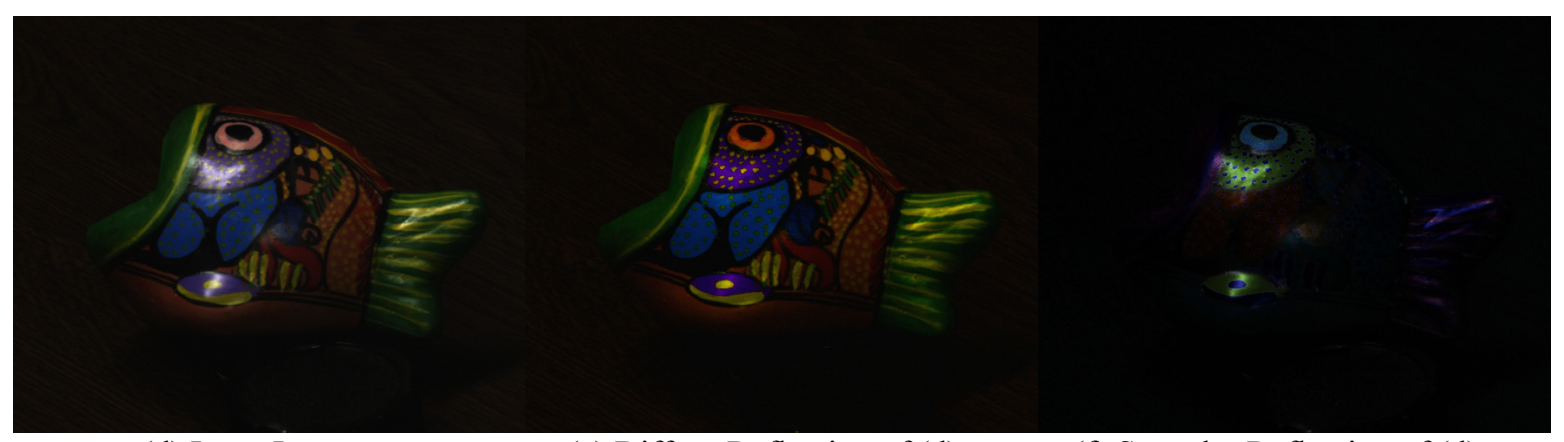

(d) Input Image

(e) Diffuse Reflection of (d)

(f) Specular Reflection of (d)

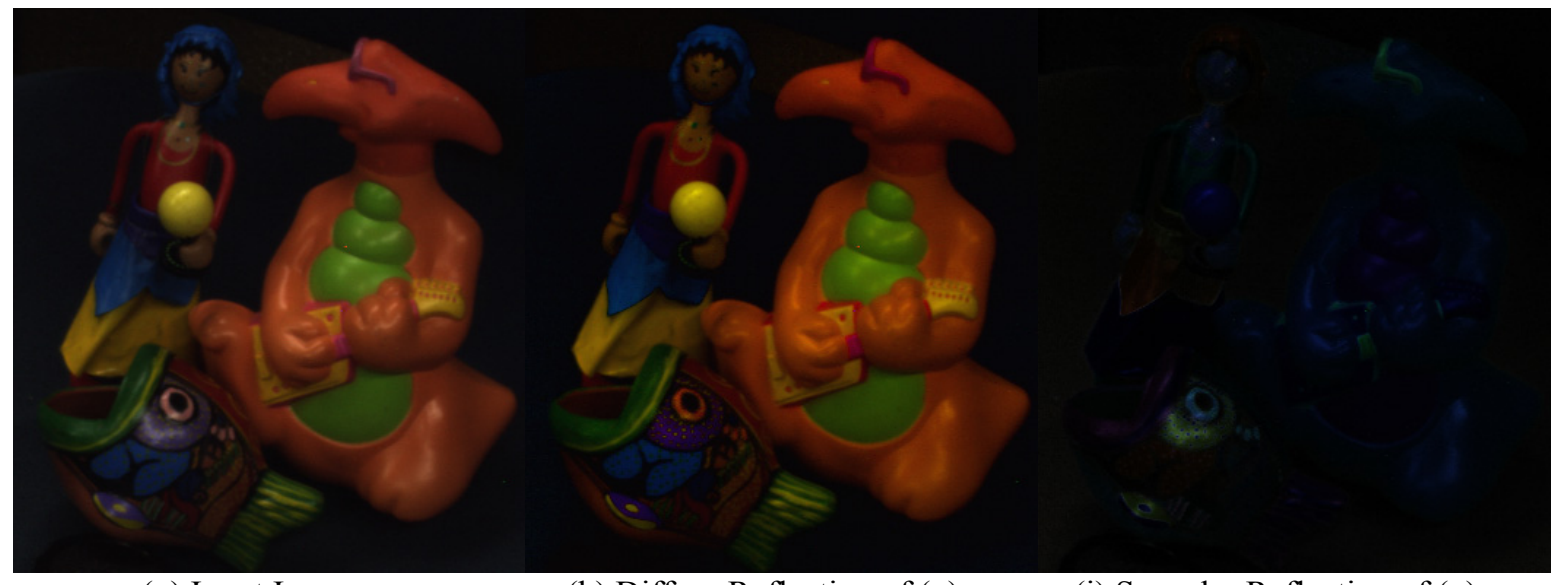

(g) Input Image

(h) Diffuse Reflection of (g)

(i) Specular Reflection of (g)

Figure 3.5 (Cont.) Reflection separation results for input images having multicolor surfaces. 


\section{CHAPTER IV}

\section{Color based Specular Reflectance Estimation for Scene Illumination Rendering}

\subsection{Introduction}

Given the importance of color processing in computer vision and computer graphics, rendering illumination spectral reflectance of image scenes is important to improve the capability of a large class of applications that include image editing, scene reconstruction, and surface segmentation, and other applications in which visual effects are simulated by independent processing of separating the diffuse and specular image layers.

An image is formed as the product of the scene's shape, illumination and reflectance. Many visual tasks rely only on a subset of this information and extract the targeted scene properties in a manner that is insensitive to the variation of the remaining ones. For instance 3D shape reconstruction is accurately designed by systems that have a significant control over the object's illumination distribution and reflectance parameters such as texture and surface roughness. Furthermore, other approaches rely on discriminating some of the scene properties by selecting image features that are invariant to the scene property chosen. This approach is often used for material based object segmentation under the assumption of a Lambertian scene model.

In this chapter, we present a scene illumination rendering technique that estimates the relative contributing specular reflectance attributes of a scene image while preserving the diffuse reflectance information. We consider a class of image feature subsets that preserve the geometrical and photometrical information of an image scene while isolating 
and rendering its illumination distribution properties exclusively. The image feature subset targeted provides a direct access to the surface illumination information. We introduce an efficient separation method based on Eigen-decomposition transformation to isolate the diffuse and specular reflectance information from color images. Solely based on the image color properties, our method operates over the inverse-intensity chromaticity space. This normalized color space has the benefit of isolating each image reflectance properties in a nearly distinct manner.

The proposed decomposition method separates both reflectance properties while maintaining their inner intrinsic features unmarked and contributing to the realistic rendering effects perceived. Moreover, we show that by mathematically reshaping the dynamic range distribution of the specular reflectance components over each color channel we render the scene illumination reflection effectively without altering the scene's surface diffuse attributes.

\subsection{Contributions}

To render illumination reflectance components in colored image scenes, we introduce the following contributions:

- Developed a method to estimate the illumination reflectance component by means of the Mean-Shift Decomposition $(M S D)$ and the Eigen-decomposition transform.

- Evaluated the distribution of the projected image intensities on the $I I C$ chromaticity space using a statistical approach on the proposed MSD method to separate both specular and diffuse reflectance values effectively. 
- Established a linear transformation method similar on these distributions in order to achieve the illumination rendering process.

- Demonstrated that the proposed rendering method can produce real world illumination effects by comparing our obtained real rendered results with many different indoor and outdoor real world illuminations spectral distributions.

\subsection{Related Work}

Several photometric methods have been proposed for Lambertian scenes. R-G chromaticity, normalized-RGB, and hue/saturation images are examples of representations that are dependent uniquely on the spectral reflectance of the surface and the spectral power distribution of the illuminant. The diffuse surface components of images that are represented by a dichromatic reflectance model [Shafer 1985] depend heavily on the material properties of the surface. However, the spectral distribution of the specular component is similar to that of the illuminant. This model suggests the possibility of decomposing an image into its specular and diffuse components based on its local color information.

Operating on image reflection components has been the focus of many investigations. Klinker et al. [Klinker, 1988] show that when the diffuse color is the same at each point on an object's surface, the color histogram of its image forms a T-shaped distribution, with the diffuse and specular pixels forming linear clusters. They exploit this information to evaluate a single generalized diffuse color. In practice, this approach can be employed in applications in which an image is segmented into several regions of homogeneous 
diffuse color. Also, significant improvement were suggested to be made by exploiting the knowledge of the illuminant color through transformations of color space [Bajcsy, 1996] [Tan, 2004(b)], but these methods also require an explicit segmentation of the scene into large regions of constant diffuse color.

Other approaches make use of local interactions in representing the reflection components. Local interactions approaches accept highly textured scenes that contain varying diffuse colors. However, most of these methods assume the illuminant color is a priori known. Tan and Ikeuchi [Tan, 2005] iteratively reduce the specular component effects by considering pixel neighbors that are assumed to have a related diffuse component. Ping et al. [Ping, 2003] allowed for a user to specify a closed curve surrounding a specular region to then minimize an objective function based on local variations in diffuse chromaticity and specular intensity. Kawakami et al. [Kawakami, 2005] suggest more restrictive assumptions to handle scenes with mixed illuminations. They assume that all shadows are hard and that illumination spectrum distributions are black body radiators. On the other hand, Ebner [Ebner, 2004] introduces a method mainly based on localized grey world assumptions. His method renders scene illumination effectively when objects are predominantly gray, but don't excel when large colored objects are introduced.

The structure of this chapter is organized as follows. We begin by introducing a general background on the image formation based on a dichromatic reflection model in Section 4.4. In Section 4.5, we review the use of the inverse-intensity chromaticity space in our proposed method. In Section 4.6, a new method for separating the diffuse and specular components in color images is presented based on the Mean-Shift Decomposition (MSD) 
method. A linear transformation method is proposed to render the specular reflection attributes depicted from the MSD method will be described in section 4.7. In Section 4.9, we evaluate our rendering results using different real-world illumination distributions to confirm the effectiveness of our proposed method. And finally in Section 4.10, we summarize our study.

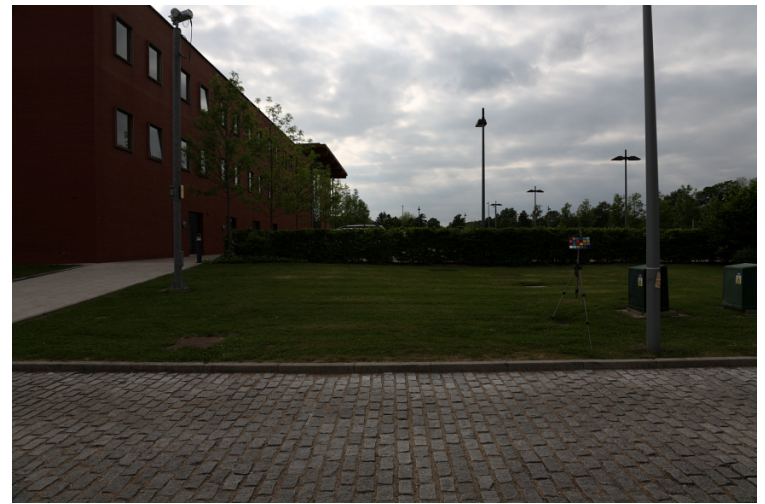

(a) Input image

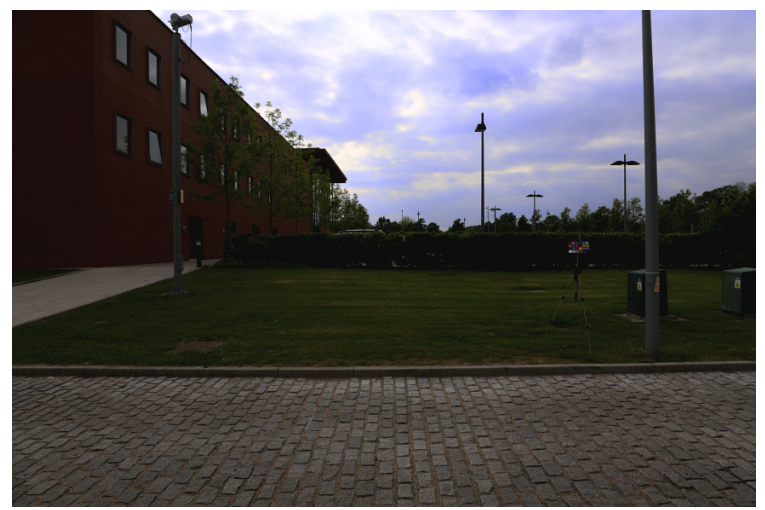

(c) Rendered input image with $(1.0,1.0,0.93)$

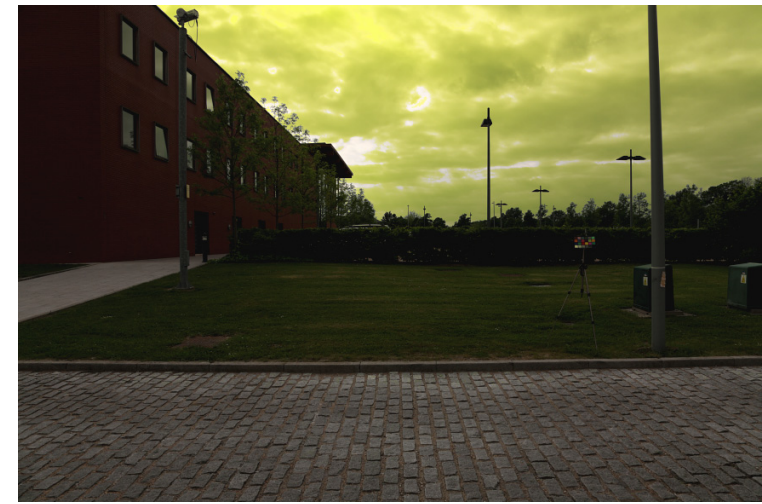

(b) Rendered input image with $(1.0,1.0,1.5)$

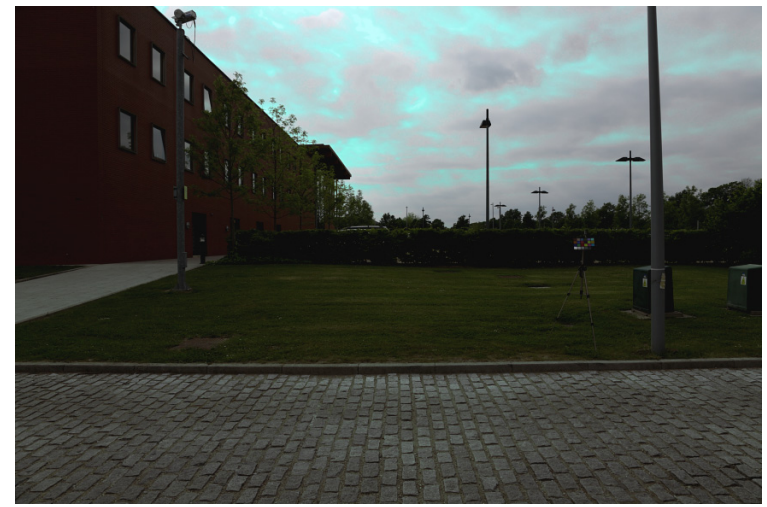

(d) Rendered input image with $(0.88,1.0,1.0)$

Figure 4.1 Image (a) shows an outdoor scene image taken under mixed illumination. Images (b-d) portray the use of our proposed method to render scene illumination using different thresholds $\left(\eta_{R}, \eta_{G}, \eta_{B}\right)$ 


\subsection{Dichromatic Reflection Model}

A Lambertian model of image formation is assumed where the illumination spectral power distribution $E(\lambda)$ is incident upon a surface and whose reflectance is $S(\lambda, n)$ corresponding to the image pixel location $n \in Z^{2}$ as a function of the electromagnetic wavelength $\lambda \in R$ over the visible spectrum $\Omega$.We also assume a uniform illumination color independent of spatial location $n$, where the tri-stimulus intensity value recorded by the camera sensors can then be expressed as:

$$
\rho_{k}(\lambda, n)=\int_{\Omega} S(\lambda, n) E(\lambda) q_{k}(\lambda) d \lambda
$$

where $q_{k}(\lambda): R \rightarrow R$ is the spectral sensitivity of the $k^{\text {th }}$ linear sensor channel, $k=1,2,3$, and $\rho_{k}(\lambda, n)=\left[\rho_{1}(\lambda, n), \rho_{2}(\lambda, n), \rho_{3}(\lambda, n)\right]$ is the triplet of sensor responses at pixel location $n$.

Shafer [Shafer 1985] proposed a reflection model represented by a linear combination of diffuse and specular reflections known as the dichromatic reflection model. According to this model, the spectral reflection distribution of the specular component at each surface location is the same as that of the illuminant, while the spectral reflection distribution of the diffuse component depends on the reflectance of the surface $S(\lambda, n)$. Let $S_{d}(\lambda, n)$ denote the surface reflectance with regard to the diffuse spectral reflection and let $S_{s}(\lambda, n)$ denote the surface reflectance with regard to the specular spectral reflectance, then the color intensity measured by the camera sensors becomes:

$$
\rho_{k}(\lambda, n)=m_{d}(n) \int_{\Omega} S_{d}(\lambda, n) E(\lambda) q_{k}(\lambda) d \lambda+m_{s}(n) \int_{\Omega} S_{s}(\lambda, n) E(\lambda) q_{k}(\lambda) d \lambda
$$


here $m_{d}(n)$ and $m_{s}(n)$ are scale factors for the diffuse and specular reflection respectively, and their values change depending on the geometric structure at location $n$. The objective of separating the reflection components is still an ill-posed problem without further assumptions. Here, we assume that the camera sensors depict an exact Dirac delta function and the illumination is restricted such that it can be modeled by Planck's law [Planck 1959]. If the camera sensor's response characteristics are not narrow-band they still can be sharpened using these algorithms [Barnard, 2001][Finlayson, 1996][Finlayson, 1994].

With these assumptions, Equation 4.2 can be rewritten as:

$$
\rho_{k}(n)=m_{d}(n) S(n)_{d, k} E+m_{s}(n) S(n)_{s, k} E
$$

for simplicity, we express Equation 4.3 as:

$$
\rho_{k}=m_{d} \phi_{d, k}+m_{s} \phi_{s, k}
$$

where $\phi_{d, k}=S(n)_{d, k} E$ and $\phi_{s, k}=S(n)_{s, k} E$.

\subsection{Inverse-Intensity Chromaticity (IIC) Space}

Similar to most existing methods for specular and diffuse separation, our approach exploits the color differences between both specular and diffuse reflections as described in the dichromatic reflection model. Based on that model, we consider the tri-stimulus value recorded by the color imaging device to be a set of RGB values so that $\rho_{k}: R^{3} \rightarrow[0,1]$ where $k \in\{r, g, b\}$. 
Rewriting Equation4.4 in terms of chromaticity, we let $\sigma_{k}=\frac{\rho_{k}}{\rho_{r}+\rho_{g}+\rho_{b}}$ represents the input intensity chromaticity, $\omega_{d, k}=\frac{\phi_{d, k}}{\phi_{d, r}+\phi_{d, g}+\phi_{d, b}}$ be the diffuse reflection chromaticity, and $\omega_{s, k}=\frac{\phi_{s, k}}{\phi_{s, r}+\phi_{s, g}+\phi_{s, b}}$ be the specular reflection chromaticity. Consequently, Equation 4.4 can be rewritten as:

$$
\sigma_{k}=m_{d}^{\prime} \omega_{d, k}+m_{s}^{\prime} \omega_{s, k}
$$

where $m_{d}^{\prime}=m_{d}\left(\phi_{d, r}+\phi_{d, g}+\phi_{d, b}\right)$ and $m_{s}^{\prime}=m_{s}\left(\phi_{s, r}+\phi_{s, g}+\phi_{s, b}\right)$

It is also important to set the device output to be linear to the flux of incoming light intensity. Tan et al. [Tan, 2004(a)] proposed a method to estimate illumination chromaticity color by deriving the correlation between specular reflection chromaticity and input intensity chromaticity delineated in a space called the inverse-intensity chromaticity space that we abbreviate as IIC-space. The relation between these two components is expressed as follows:

$$
\sigma_{k}=\alpha \frac{1}{\sum_{j=r, g, b} \rho_{j}}+\omega_{s, k}
$$

where $\alpha=m_{d}^{\prime}\left(\omega_{d, k}-\omega_{s, k}\right)$

A complete derivation of Equation 4.6 can be found in [Tan, 2004(a)]. 
This equation has an important aspect in our proposed method toward the rendering of the surface specular illumination. The process of separating the diffuse and specular components for both uniformly colored and multicolored objects or scenes relies on identifying the location of each reflection elements of the image within the IIC-space. As shown in Figure 4.2, by projecting a colored surface on the IIC-space, the diffuse and specular image pixels form two clusters are partially independent as can be seen in Figure 4.2.b.

For Equation 4.6 to be linear, it is necessary to consider the value of $\boldsymbol{\alpha}$ to have a constant value, which is not always the case since its value is associated with the geometrical attributes of the surface diffuse reflection components that vary according to each object surface properties. We carry out our method based on this fact to estimate and separate efficiently both the diffuse and specular reflectance image features, which is viewed as an important pre-processing step toward the rendering of the specular illumination reflections. 


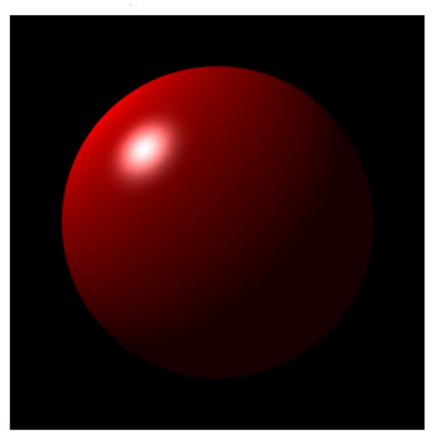

(a)

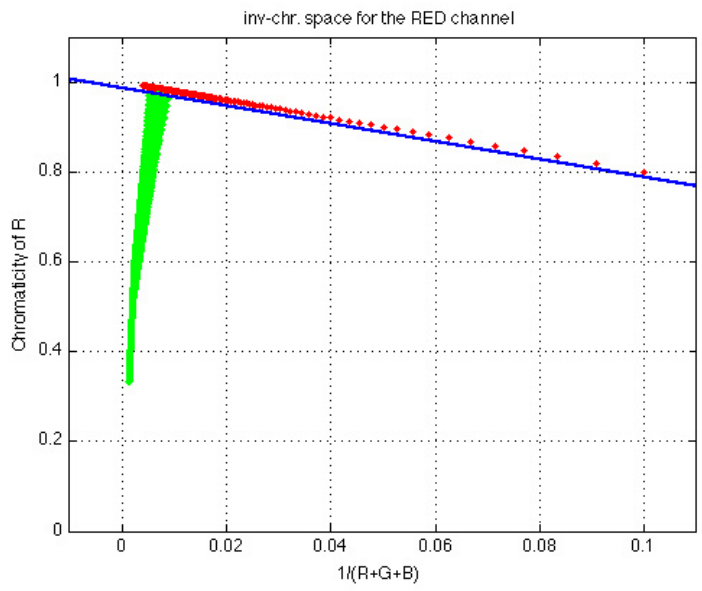

(d)
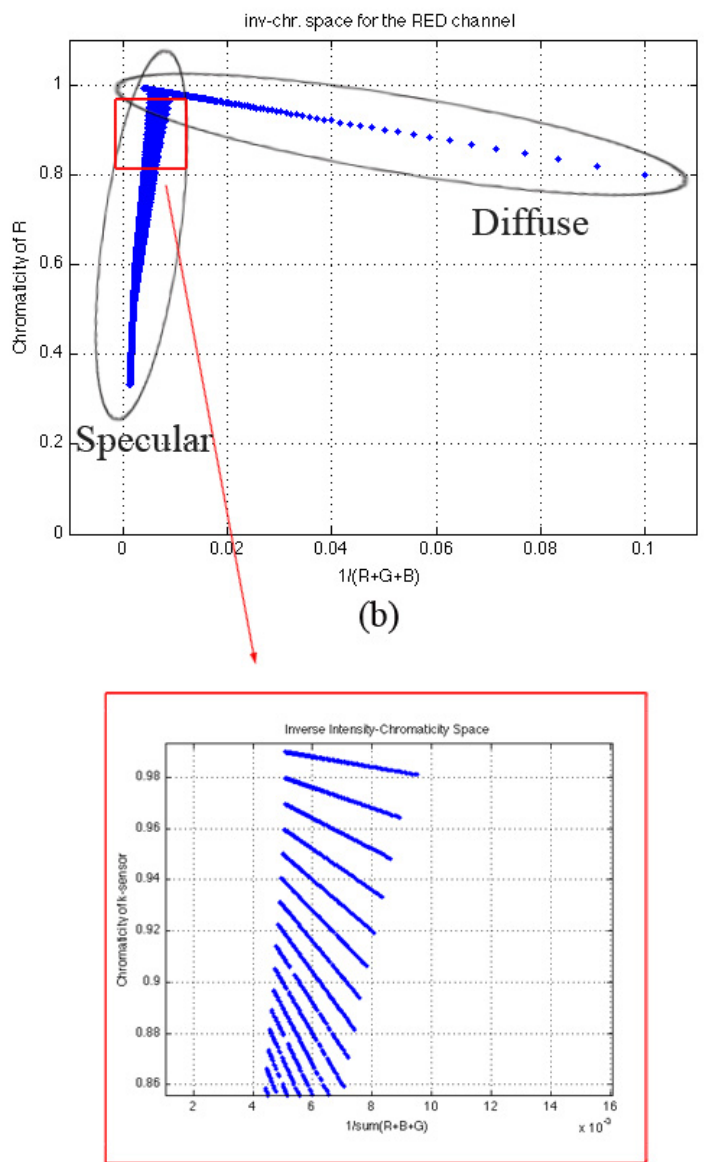

(c)

Figure 4.2 An overview of the proposed reflectance separation method. (a) Synthetic input image. (b) Diffuse and specular points of the projected input image on the IIC-space. (c) A magnification of the portrayed projected image chromaticity values forming clusters of straight lines. (d) Resulted reflectance cluster separation using the proposed decomposition method. The blue line represents the separation line while the green and red clusters represent the specular and diffuse reflectance components respectively.

\subsection{Surface Reflectance Decomposition}

Some previous methods propose solving the reflectance separation problem by requiring the manual identification of highlights in surface regions, restrictive assumptions about the scene surface to be textured or untextured, or the use of physical filters e.g. polarizing 
filters [Mallick, 2006]. The advantage of processing dichromatic surfaces on a chromaticintensity space is the ability to preserve the intrinsic properties of the object surface. Our method has the benefit of using solely image color information without involving any local image segmentation techniques. We begin our method by introducing a partial specular/diffuse separation approach to group both reflectance components into two distinct sets of pixel values. Then, by reshaping the dynamic range of the specular cluster for each color channel, we show that the specular reflection component remodel the illumination effect over an image scene gradually while hardly affecting the surface diffuse reflection constituents.

Our objective is to find a separation line that approximates the location of the diffuse and specular reflectance clusters robustly. Many techniques can be adopted for this task [Cheng, 1995] [Kaufman, 1990] [Nishino, 2001]. However, since our method tends to be repetitive along all image channels, we opted for a simple yet efficient solution that linearly differentiates the two sets of reflectance. We thus introduce a separation method based on Eigen-decomposition that we call Mean-Shift Decomposition (MSD). As with any other Eigen-decomposition technique in multivariate data analysis, the mean-shift decomposition method attempts to find the correlated features associated with each reflectance component mainly promoted in the center of the data, Figure 4.2.b. A correlation matrix is specifically generated to detect the relationship between both reflectance components. A change in the position of the mean of both reflectance components synchronizes the classification of each reflectance feature within its type labeled to be as either a diffuse feature or a specular feature. 
We consider the correlation matrix $C$ of each vector set represented in the IIC-space to be composed of the correlation coefficients $C_{i} \in[-1,1]$ between the vectors as $i=1, \ldots, N$. The eigenvalues $\lambda_{l}$ and the eigenvectors $v_{l}$ are defined formally using the standard equation:

$$
C v_{l}=\lambda_{l} v_{l}
$$

for $l=1, \ldots, N$. Generally for a vector size of $i=1, \ldots, N$ Equation 4.7 has $N$ different solutions. We assume that the eigenvectors are normalized and the solutions have been sorted according to the eigenvalues $\lambda_{1} \geq \lambda_{2} \geq \ldots \geq \lambda_{N}$.

Also, transforming a matrix from its eigenvector basis, the matrix diagonal is represented by its eigenvalues and its trace remains invariant, therefore for the correlation matrix to be valid, the eigenvalues need to satisfy the relation $\sum \lambda_{l}=\operatorname{tr}(C)=N$.

The mean-shift decomposition method separates classes forming a set of data attributes by synchronizing the means of the projected vector sets based on the computed eigenvectors directions. Here we consider a two-class problem where the classes are labeled to contain either diffuse reflection components or specular reflection components. The means of the two vector sets are given by the standard formulations of $\mu_{x}=\frac{1}{N} \sum_{n=1}^{N} \frac{1}{\sum_{j=r, g, b} \rho_{j, n}}$ and $\mu_{y}=\frac{1}{N} \sum_{n=1}^{N} \sigma_{k, n}$. A simple separation measure of the two classes would be the separation of the projected means along a weight vector $w$. Doing so, we need to choose $w$ to satisfy

$$
\mu_{x}-\mu_{y}=\underset{w}{\operatorname{argmax}}\left(w^{T}\left(\mu_{x}-\mu_{y}\right)\right)
$$


However, this expression varies and can be made arbitrary large simply by increasing the magnitude of $w$. We still can constrain the value of $w$ to have a unit length such that $\sum_{i} w_{i}^{2}=1$. But a problem will rise when determining the orientation of the line that separates the two means. This mostly happens if the covariance of any of the two class distributions is not diagonal. Thus, a function that will give a large separation between the projected means while also giving a small variance within each class is important to minimize the reflectance separation line orientation error.

From Figure 4.2.c, we note that the projected data over the IIC-space form a cluster of straight lines aligned in such a way that they intersect at a certain point on the image chromaticity axis. From Equation4.6, considering the case where $\alpha$ is constant, the oriented straight lines intersect at a single point identical to the illumination chromaticity $\sigma_{k}=\omega_{s, k}$ accordingly when $\frac{1}{\sum_{j=r, g, b} \rho_{j}} \cong 0$. Based on this fact, the line separating the diffuse reflectance chromaticity and specular reflectance chromaticity should be oriented according to the relation of the lines depicted by the projected data cluster.

We chose the gradient of the line that separates the two class reflectance chromaticity to be the eigenvector of the highest eigenvalue calculated from the covariance matrix. This causes the separation line to be directed toward the angle that significantly describes the weight of each reflectance class while being proportional to the distribution of the data cluster. The intersection of the projected set means is then used to shift the line location resulting in separating the projected data cluster into two partitions Figure 4.2.d.

We describe the expression of the MSD separation line as follows: 


$$
\sigma_{k}=\bar{v}_{i}\left(\frac{1}{\sum_{j=r, g, b} \rho_{j}}-\mu_{x}\right)+\mu_{y}
$$

where $\bar{v}_{i}$ represents the eigenvector with the highest eigenvalue of the covariance matrix. This decomposition method has the advantage of keeping the intrinsic pixel relationship combining the specular and diffuse image components mutually which differs from the concepts used for object segmentation methods. In Figure 4.7-9, we show the use of the MSD technique on different scene situations.

\subsection{Specular Reflection Range Modeling}

This section proposes a new method for rendering the specular illumination reflectance in images. The image intensity values mapped on the IIC-space are transformed by rotating the coordinates of the IIC-space by projecting the inverse-intensity vector set with the direction of the effective line partitioning the two cluster reflectance. We consider an approach similar to the contrast stretching transformation concept to exploit the shape of the specular reflection cluster. Prior to that, it is necessary to identify the partitioned cluster comprising the image specular components. We choose a simple reasoning for determining the distribution of the reflectance intensities in images, especially targeting the specular reflection image pixels.

\subsubsection{Specular Reflection cluster Identification}

The linear partition method described in the previous section separates the projected image chromaticity sets into two distinct sets of clusters. However, up to this point, the 
cluster describing the constituents of the surface diffuse reflectance or surface specular reflectance component is still undecided. In Figure 4.2.b, the reflectance chromaticity values labeled were observed based on the mapping of each specular reflection component into the inverse-intensity chromaticity space independently. This allows us to consider generally where the reflection values are sited. In practice, and especially for complex scenes as in Figure 4.1, the distributions of the mapped image chromaticity values on the IIC-space are scattered and change orientation, therefore following the model presented in Figure 4.2.b will result in the incorrect reflectance classification.

Simple reasoning can be used to locate the cluster consisting of specular reflection values. We consider first the location of the specular pixels of the intensity color image. Since the specular points are among the brightest pixels as inferred from the dichromatic surface object of a Lambertian model, we determine the location of the brightest blobs in the intensity color image. Mapped to the IIC-space, those pixels are mostly inclined toward values with intensity chromaticity nearing

$$
\sigma_{i, k}=\frac{1}{\max \left(\sum_{j=R, G, B} \rho_{i, j}\right)}
$$

The objective of locating the cluster that contains the specular reflectance elements is consequently reduced to the process of locating only the most prominent specular pixels of the intensity color image before being mapped on the inverse-intensity chromaticity space. The presence of these pixels in one of the two-reflectance clusters identifies the cluster holding the specular components. 


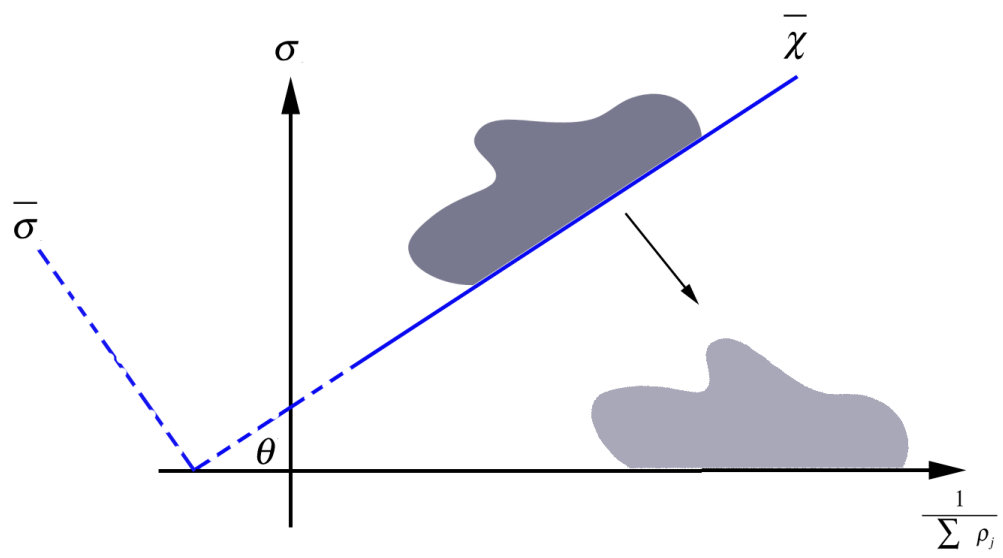

(a)

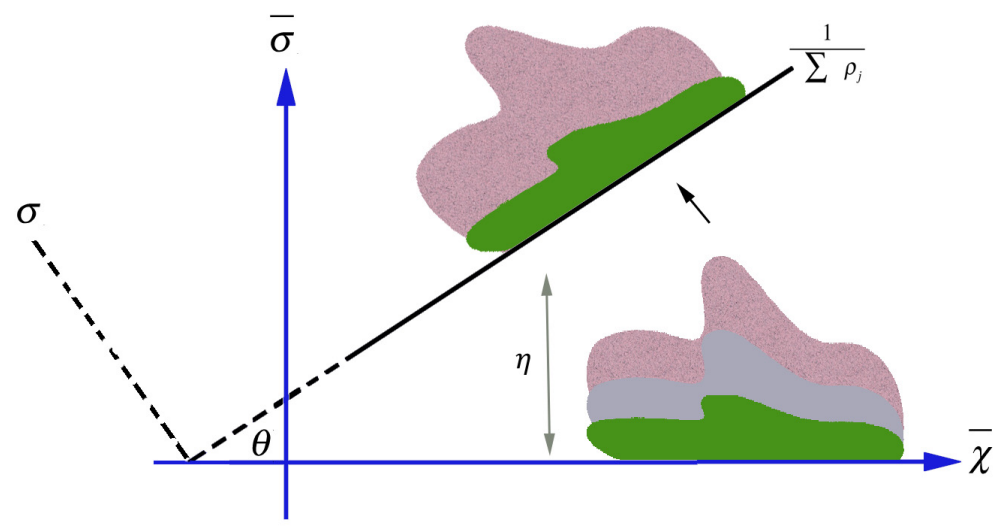

(b)

Figure 4.3 (a) Transformation of the specular components (grey) coordinates by aligning the inverseintensity axis of the IIC space with the reflection separation line (blue). (b) Specular components stretching process indicated by varying the threshold ratio $\eta>1$ (red) and $\eta<1$ (green).

\subsubsection{Specular Reflection Modeling}

The ability of rendering the illumination scenes or objects reflectance surfaces with different illumination effects resides in altering the dynamic range of the located image specular component intensities. The desired visual illumination effects are achieved by stretching the chromaticity intensities dynamic range of each channel by a threshold ratio $\eta=\left\{\eta_{R}, \eta_{G}, \eta_{B}\right\}$ such that the dynamic range of the chromaticity intensities decreases 
when $\eta<1$ or increases when the threshold ratio is $\eta>1$.

We begin by aligning the IIC inverse-intensity basis with the direction of the effective reflectance cluster line separation expressed in Equation 4.9, as displayed in Figure 4.3.a. To facilitate this projection, we assume that the origin of the new transformed coordinate system is the intersection point between the reflectance separation line and the inverseintensity sum axis. Let $\theta$ be the angular quantity representing the clockwise rotation angle between the two coordinate systems.

We define the transformed IIC coordinate system as follows:

$$
\begin{aligned}
& \bar{\sigma}_{k}=\sigma_{k} \cos (\theta) \\
& \bar{\chi}=\frac{1}{\sum_{j=R, G, B} \rho_{j}} \cos (\theta)
\end{aligned}
$$

where $\bar{\sigma}$ and $\bar{\chi}$ are the new coordinate basis of the projected IIC space representing the projected image chromaticity and the inverse sum of intensity sum of the specular components vectors, respectively.

The motivation behind the proposed basis transformation is to facilitate the linearization of the reflection data stretching process needed for illumination modeling. We derive an approach similar to the contrast stretching transformation concept [Arici, 2009][Ji, 1994][Rahman, 2004] to exploit the shape of the specular reflection cluster. As shown in Figure 4.3.b the cluster forming the specular components changes in size depending on the value of threshold $\eta$. We adopt a linear stretching mechanism to remodel the shape of the specular cluster. 
Let $\bar{\sigma}_{\min }=\min (\bar{\sigma})$ and $\bar{\sigma}_{\max }=\max (\bar{\sigma})$ be the minimum and maximum limit values of the projected intensity chromaticity set respectively.

We formulize the stretching mechanism with the following expression:

$$
\bar{\sigma}_{\text {new }}=\eta\left(\bar{\sigma}-\bar{\sigma}_{\min }\right)+\xi\left(\bar{\sigma}-\bar{\sigma}_{\max }\right)
$$

where $\quad \xi=\frac{\bar{\sigma}_{\min }}{\bar{\sigma}_{\max }-\bar{\sigma}_{\min }}$

note that when $\bar{\sigma}_{\min }=0$ the above expression is simply

$$
\bar{\sigma}_{\text {new }}=\eta \bar{\sigma}
$$

From Equation 4.13, we consider the adaptation of both the maximum and minimum values of the specular cluster component relative to the current calculated value. Also, we note that the stretching mechanism occur exclusively on the projected intensity chromaticity vector set without requiring any changes on the inverse-intensity axis.

\subsection{Algorithm Implementation}

We begin by computing the image chromaticity values used to transform the RGB color space to the inverse-intensity chromaticity space using Equation 4.5. For each color channel $k$, we map the image chromaticity values into the ICC-space using Equation 4.6 as shown in Figure 4.2.b. This space has the benefit of correlating the image chromaticity and illumination chromaticity values linearly. Therefore, given this representation, the problem of separating the specular and diffuse reflectance reduces to one of identifying the location of solely one reflectance attribute. To decompose the reflectance components 
into two distinct clusters, Figure 4.2.d, we compute the eigenvectors of the highest eigenvalue defined from the covariance matrix determined from the image chromaticity vectors and the inverse-sum vectors. Then, we use Equation 4.9 to characterize the separation line. Upon separating the two reflection clusters it is important to designate which cluster represents the specular reflectance. We use Equation 4.10 to calculate the brightest values in the image. Mapping these values into the IIC-space reflects the presence of the specular components thus locating the specular reflection cluster. The rendering process resides in altering the values of the specular reflection components.

The process begins by aligning the IIC inverse-intensity basis with the direction of the effective reflectance cluster line separation expressed in Equation 4.9, and as displayed in Figure 4.3.a. To facilitate this projection, we assume that the origin of the new transformed coordinate system is the intersection point between the reflectance separation line and the inverse-intensity sum axis. We use Equation 4.11 and 4.12 to represent the new transformed coordinate system. This axis transformation is presented to facilitate the linearization of the reflection data stretching process needed for illumination modeling. Finally we use Equation 4.13 to remodel the specular component values using the threshold $\eta$. We repeat this process to all image color channels where for each case the threshold $\eta$ is used to adjust the specular reflectance stretching process needed to achieve the desired rendering effect.

\subsection{Experimental Results}

To verify that the proposed algorithm efficiently renders scenes with different illuminants, we evaluate our method using the SFU Mondrian color dataset [Barnard, 2002]. The 
Macbeth ColorChecker ${ }^{\circledR}$ images, used in our experiment, comprise 24 different colored patches. The images were taken under eleven uniform illuminants simulating eight different daylight spectra and three fluorescent spectra using a SONY DXC-930 digital camera having a progressive $3 \mathrm{CCD}$ sensors by setting the camera gamma correction option to off. Under each illuminant the corresponding camera responses were registered as well as the input energy spectra. For evaluation, we compared the results with the average values of image chromaticity of the white reference patch captured by the camera. Other sets of images are also used to test our method. We use images taken from the color constancy dataset presented in [Gehler, 2008] and other images that were randomly selected from the Internet. It is important to mention that our image formation model assumes linear image values. For processed files such as JPEG compressed images, it is necessary to linearize the image gamma by inverting the transformations applied by the camera settings and/or software. We recommend to use linear images obtained directly from digital camera raw files since, in some cases, the process of linearizing images introduces some artifacts due to quantization and pixels saturation.

In Figure 4.4, we illustrate the results of our proposed rendering method. An illumination invariant color image was generated using a well-used color constancy algorithm [Gijsenij, 2010]. This image, Figure 4.a, has the benefit of being invariant to illumination changes and depicts the accurate surface color reflectance taken during the image registration process. We use this corrected image as our ground truth image for the remaining of the illumination-rendering test. For each of the 11 illuminants, we estimate the specular reflectance component based on the average value of the real-illuminant white reference image chromaticity. The threshold values used in Equation 4.13 are 
approximated to render each input image channels of the white reference patch. This simulates the effects generated by the real-illuminants (as shown in the Estimated Image column). The image difference of both the input image and estimated illumination rendered input image with respect to the ground-truth image are considered to reflect the differences between the two illuminated images. As shown, most of the diffuse reflectance components generated from our method are invariant to the illumination changes. Furthermore, the specular reflectance components do approximate the image illuminant effects of each of the daylight illuminants and the tungsten illuminants.

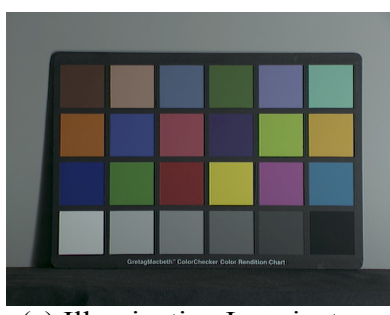

(a) Illumination Invariant Input Image Input Image
Input Image Difference
Figure 4.4 Redering the Macbeth ColorChecker ${ }^{\circledR}$ image (a) with different illumination effects generated using the proposed method. Note the specular components of the input image are affected while the diffuse components stay unchanged.

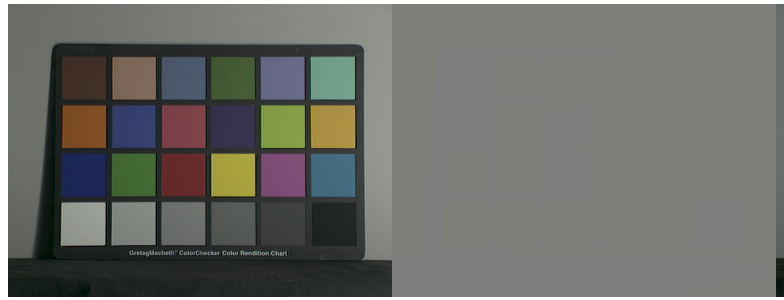

(b) Philips-Ultralume (b) - (a)

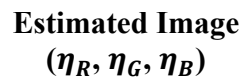

Est. Image Difference

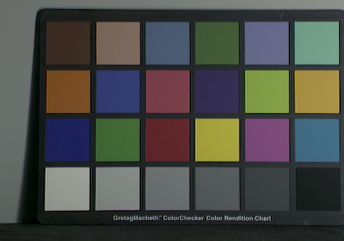

(c) Estimated Philips-Ulm (1.0114, 0.9998, 0.9867) (c) - (a)

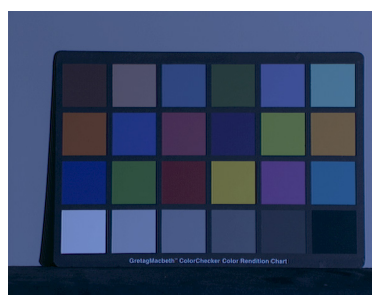

(d) Solux 3500 (d) $-(a)$

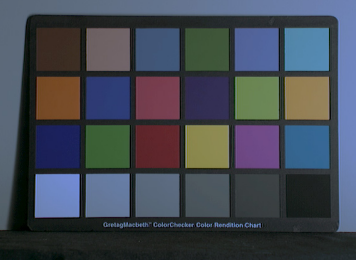

(e) Estimated Solux 3500 (1.0488, 1.0422, 1.0639) (e) $-(a)$ 


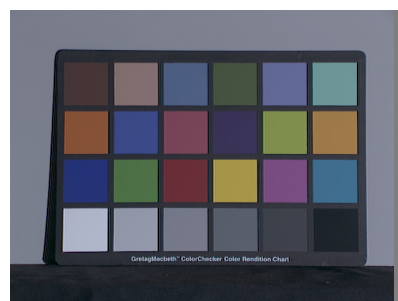

(f) Solux 3500+3202 (f) - (a)

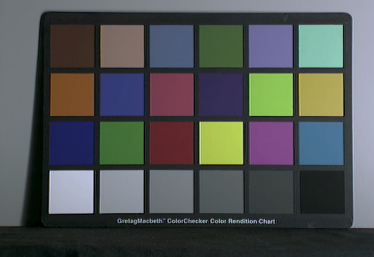

(g) Estd. Solux 3500+3202 $(0.8963,0.9541,1.0591)$

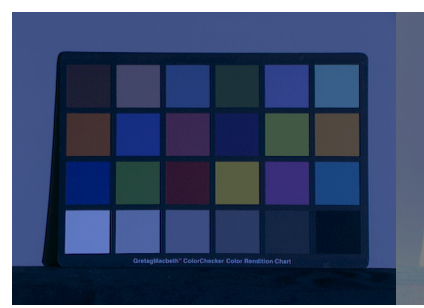

(h) Solux 4100

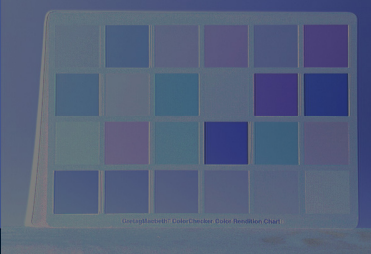

(h) - (a)

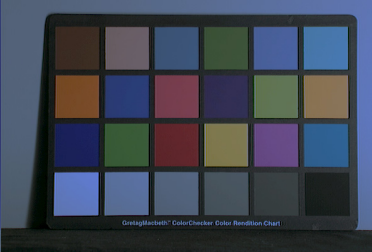

(i) Estimated Solux 4100 (1.0113, 1.0147, 1.0616)

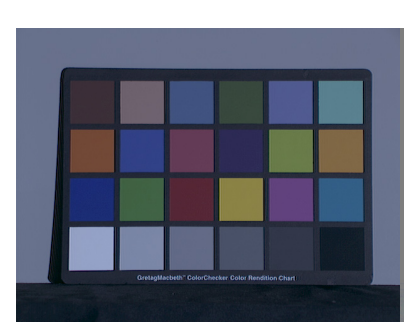

(j) Solux 4100+3202 (j) - (a)

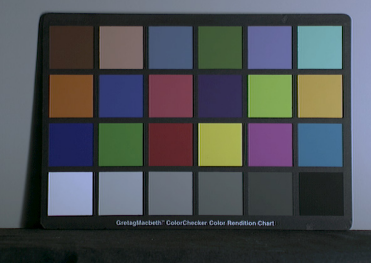

(k) Estd. Solux 4100+3202 $(0.8001,0.9011,1.0562)$

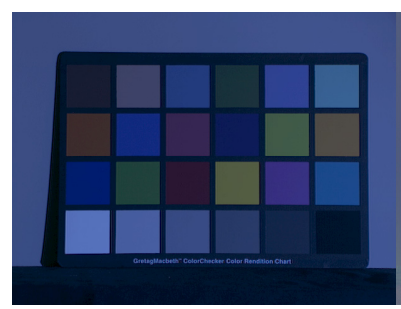

(1) Solux 4700

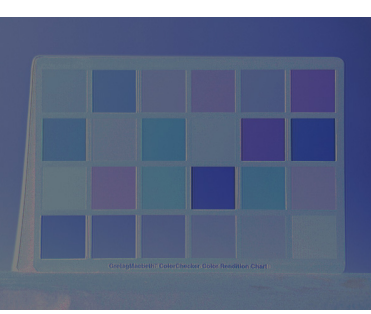

(1) - (a)

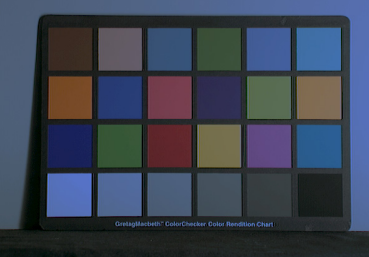

(m) Estimated Solux 4700

$(0.9885,1.0092,1.0640)$ (i) - (a) (g) - (a)

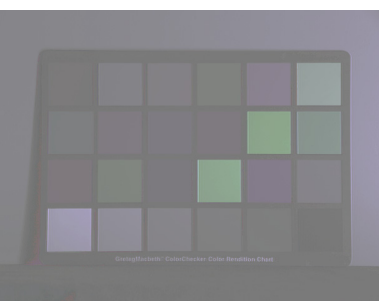

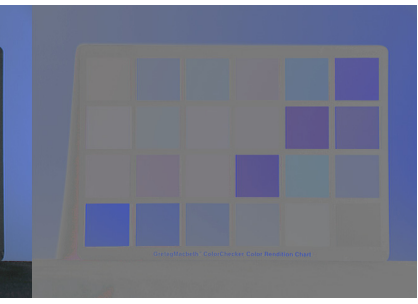




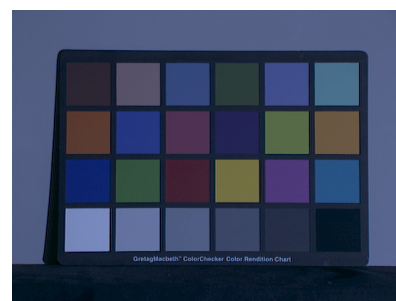

(p) Sylvania 50MR16Q (p) - (a)

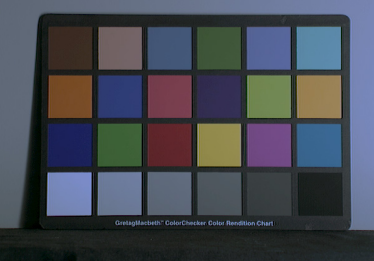

(q) Estd. Syl 50MR16Q

$(1.0609,1.0417,1.0550)$

(q) - (a)

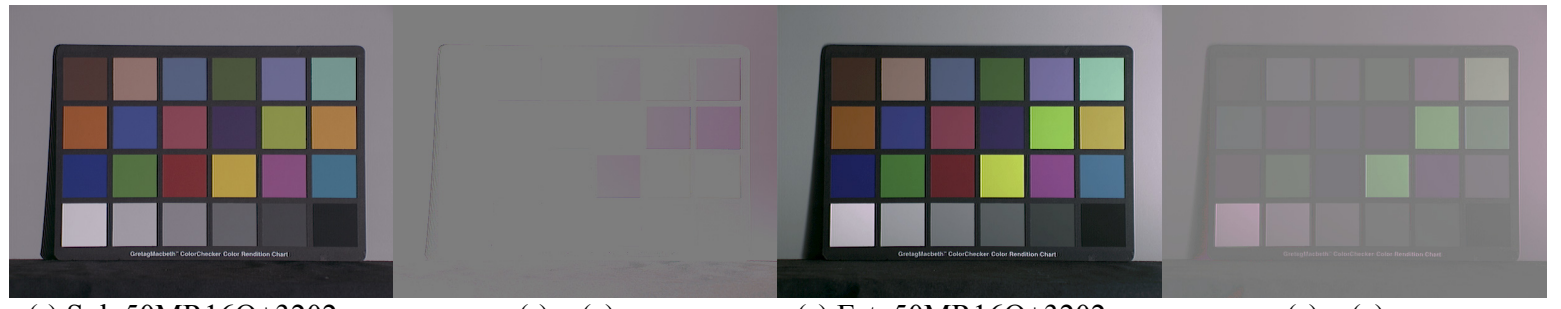

(r) Syl. 50MR16Q+3202

(r) - (a)

(s) Est. 50MR16Q+3202

$(0.9324,0.9573,1.0515)$

(s) - (a)

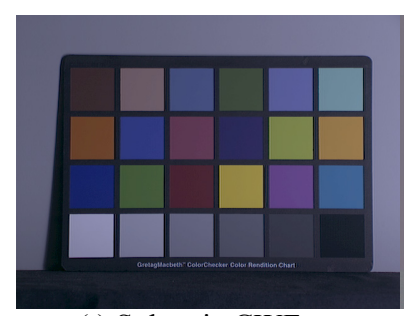

(t) Sylvania CWF

(t) $-(a)$

(u) Estimated Syl. CWF

(0.9811, 0.9571, 1.0297)

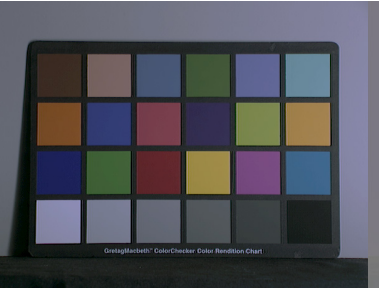

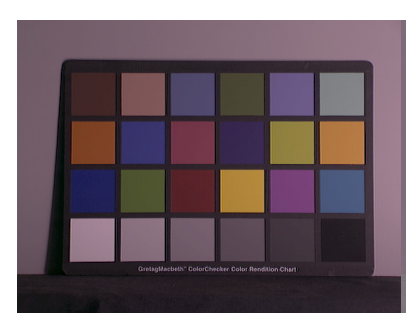

(v) Sylvania WWF

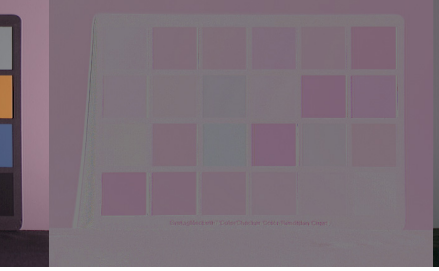

(v) - (a)

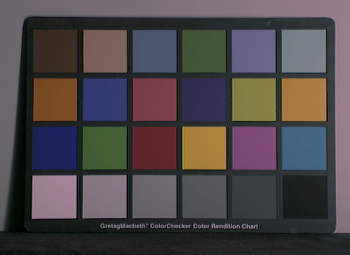

(w) Estimated Syl. WWF

$(1.0248,0.9332,0.9842)$ (u) - (a) 
the input illuminated images and the estimated rendered images since the camera CMFs were not provided with the dataset. Figure 4.5 shows the results comparing the estimated spectral power distribution for each of the 11 illuminants with the real spectral power distribution estimated.

We measure the performance of the proposed illumination rendering method using the mean error measurements of both experiments portrayed in Figure 4.4 and Figure 4.5 as shown in Table 4.1. The illumination chromaticity-mean error (CME) calculates the average error between the estimated image specular components of the rendered image and the input illuminated image. Similarly, the illumination spectral error presents the average error of the estimated spectral power distribution of the rendered image with respect to the spectral power distribution of the illuminated input image.

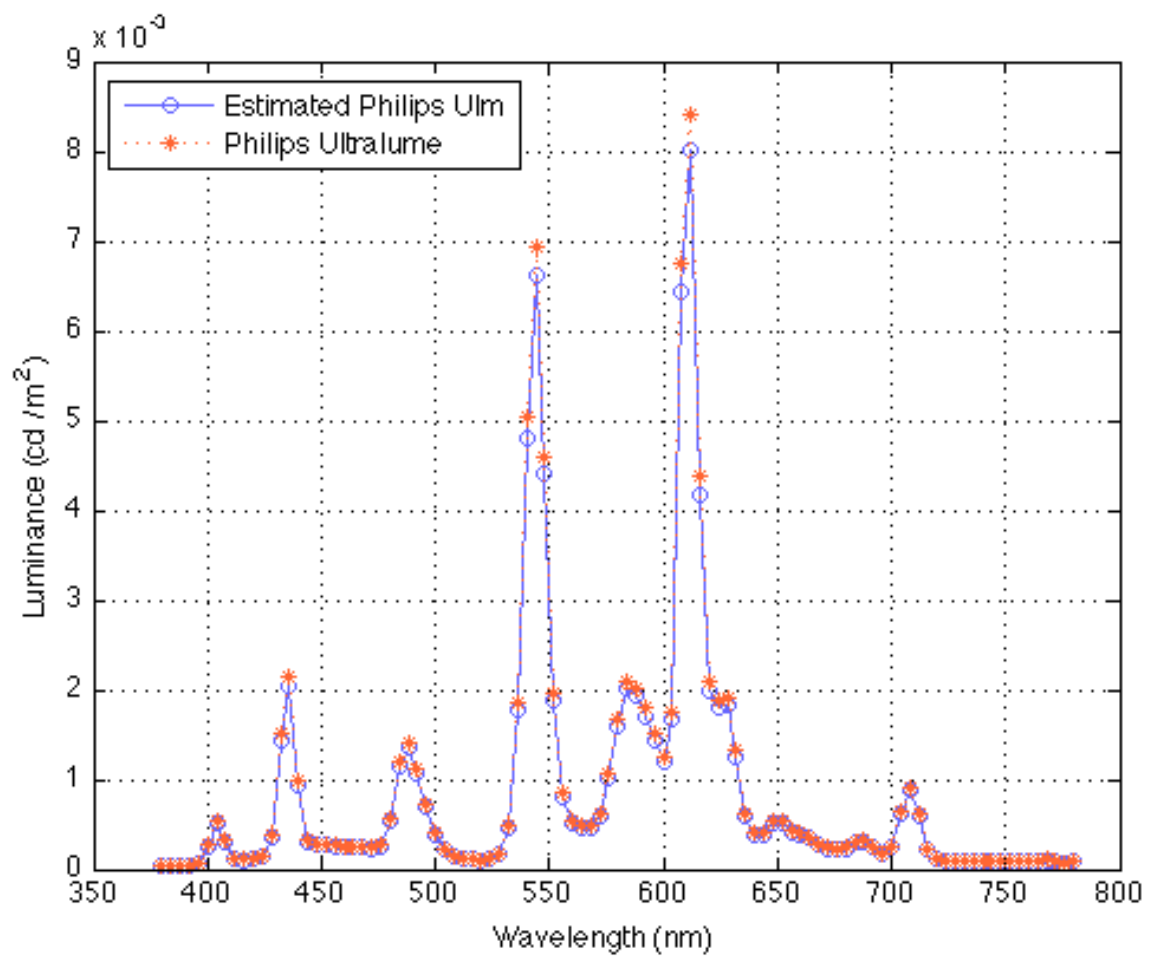

(a) Estimated vs. Original Philips Ultralume Illuminant 


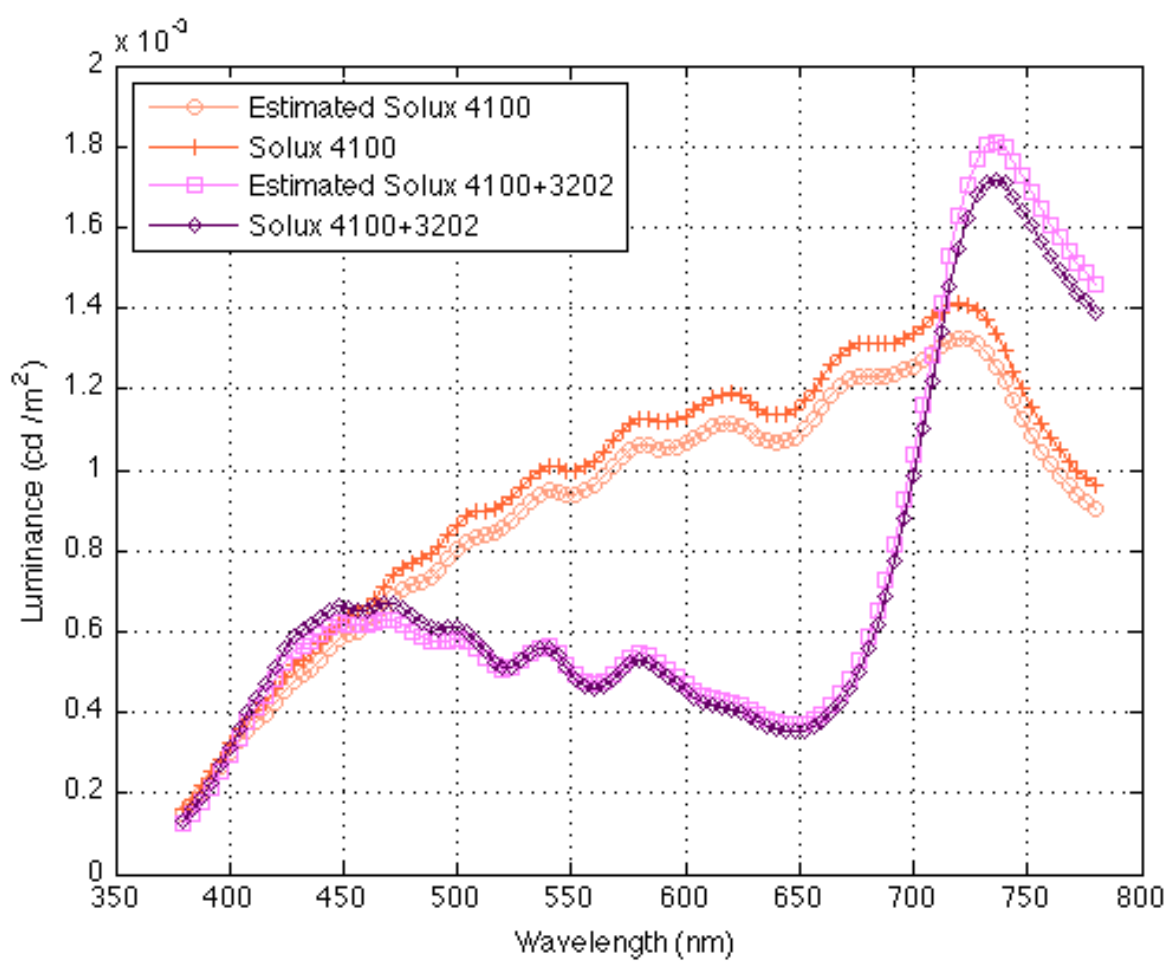

(b) Estimated vs. Original Solux 4100 and 4100+3202 Illuminant

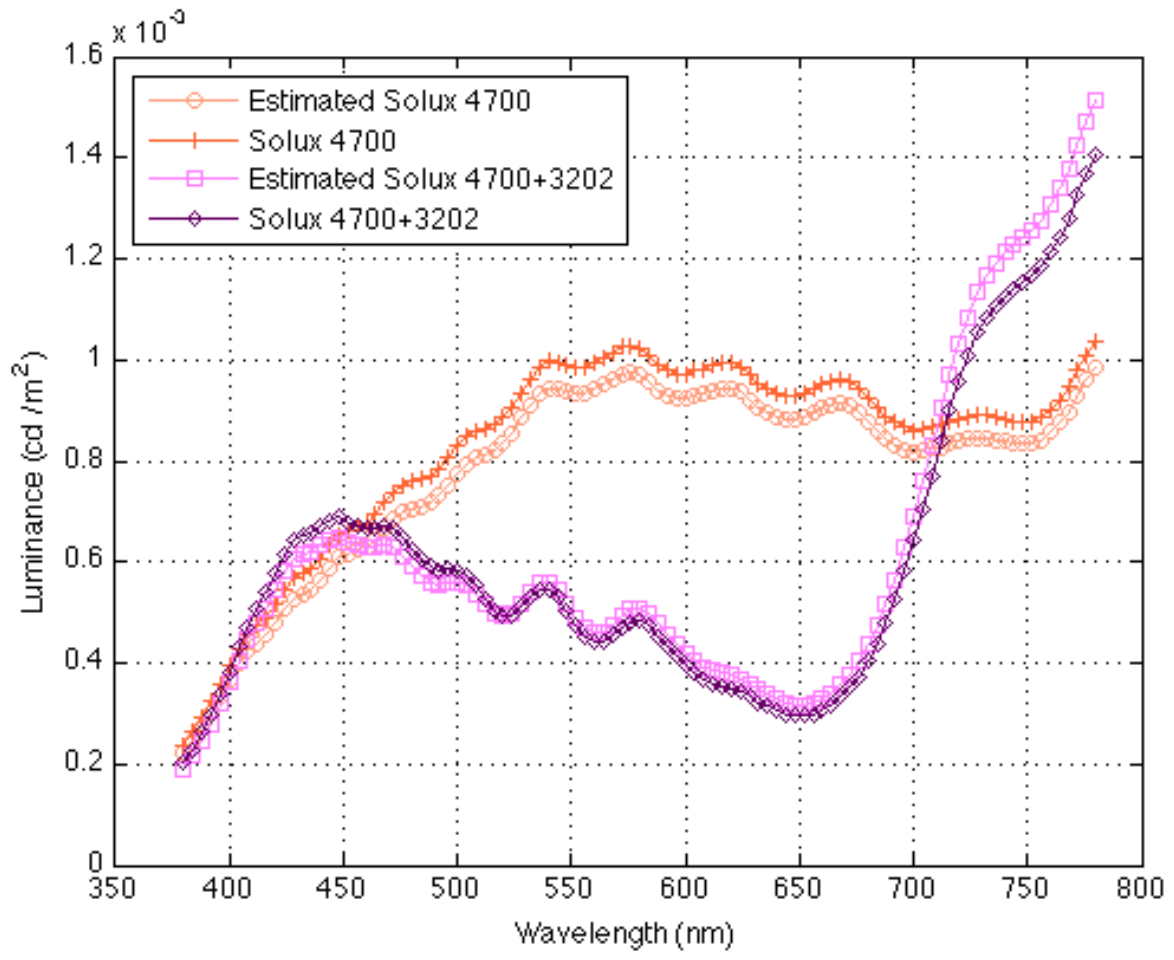

(c) Estimated vs. Original Solux $4700 \& 4700+3202$ Illuminant 


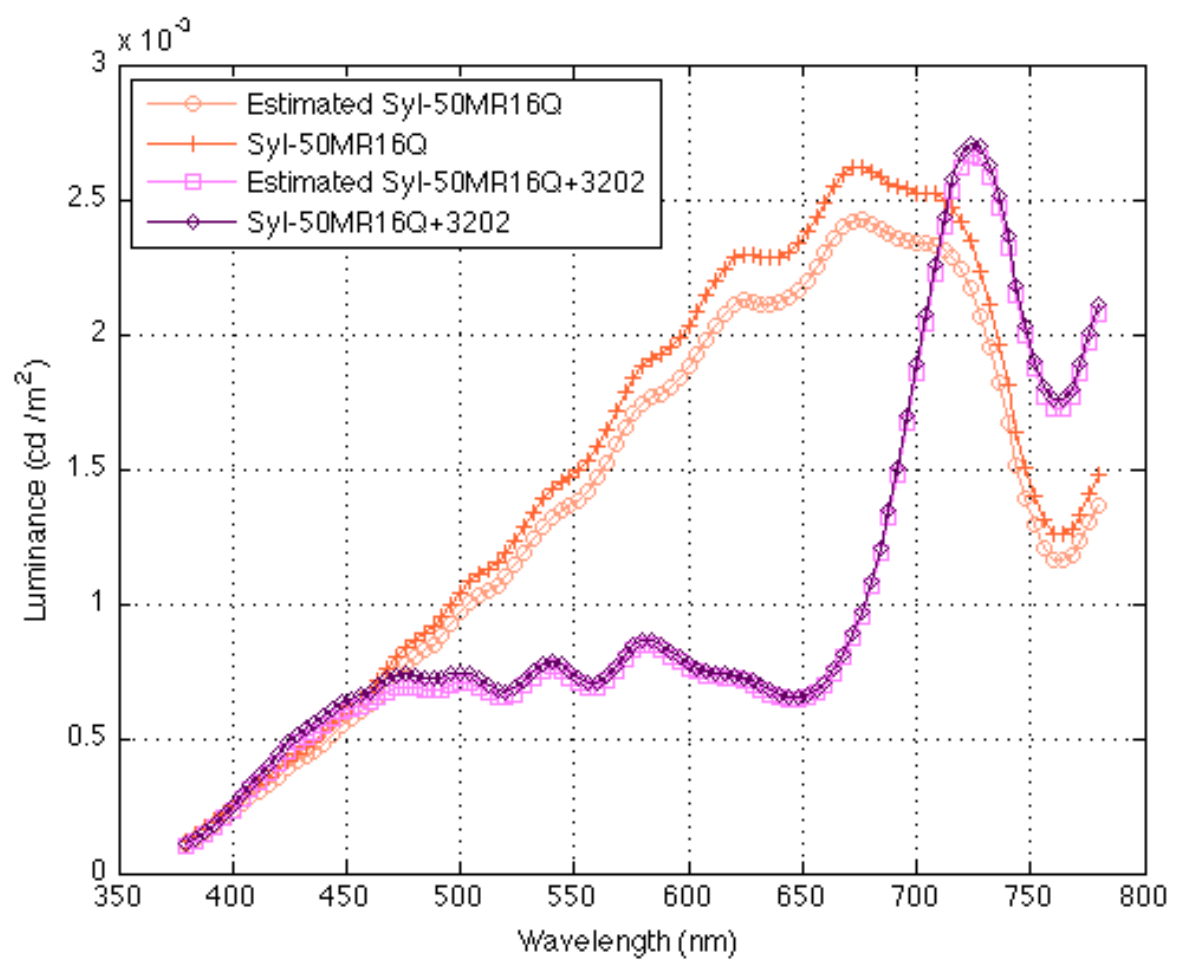

(d) Estimated vs. Original. Syl-16Q \& 16Q+3202 Illuminant

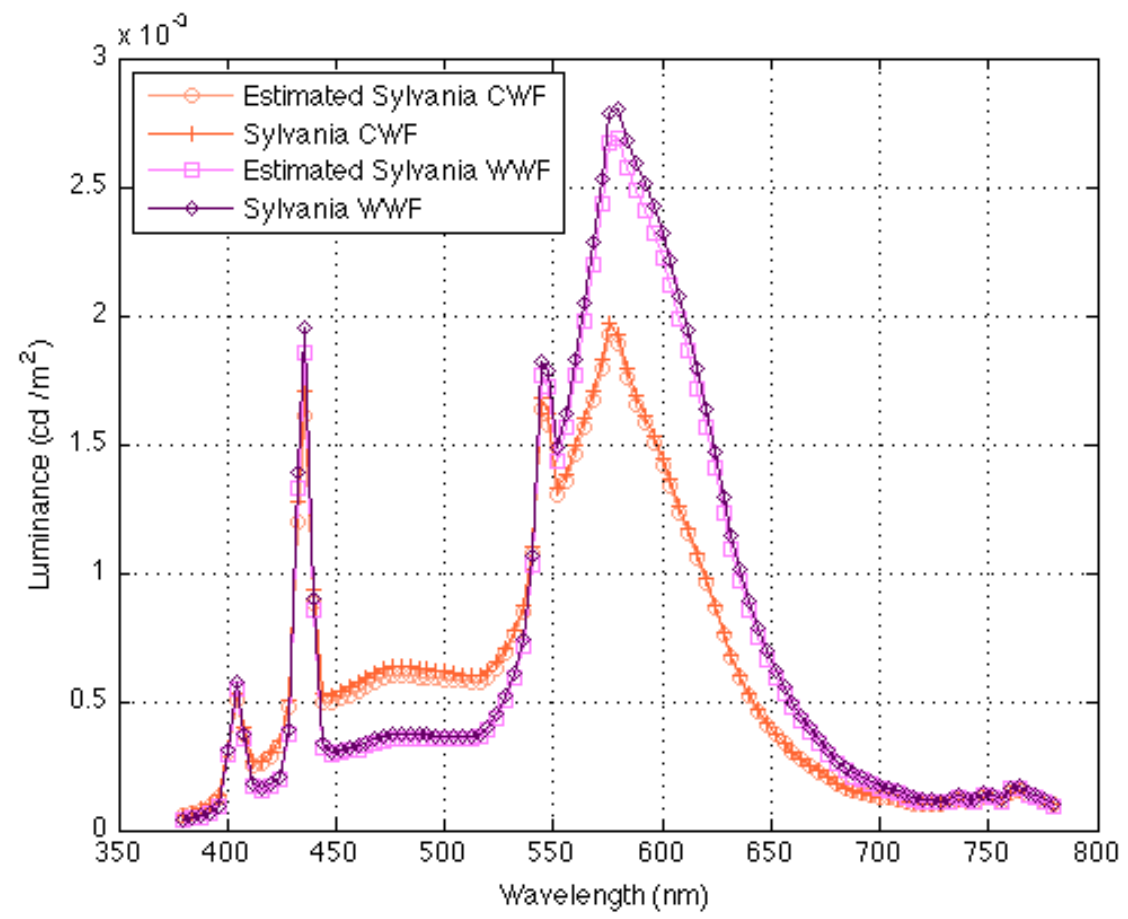

(e) Estimated vs. Original Syl-CWF \& WWF Illuminant 


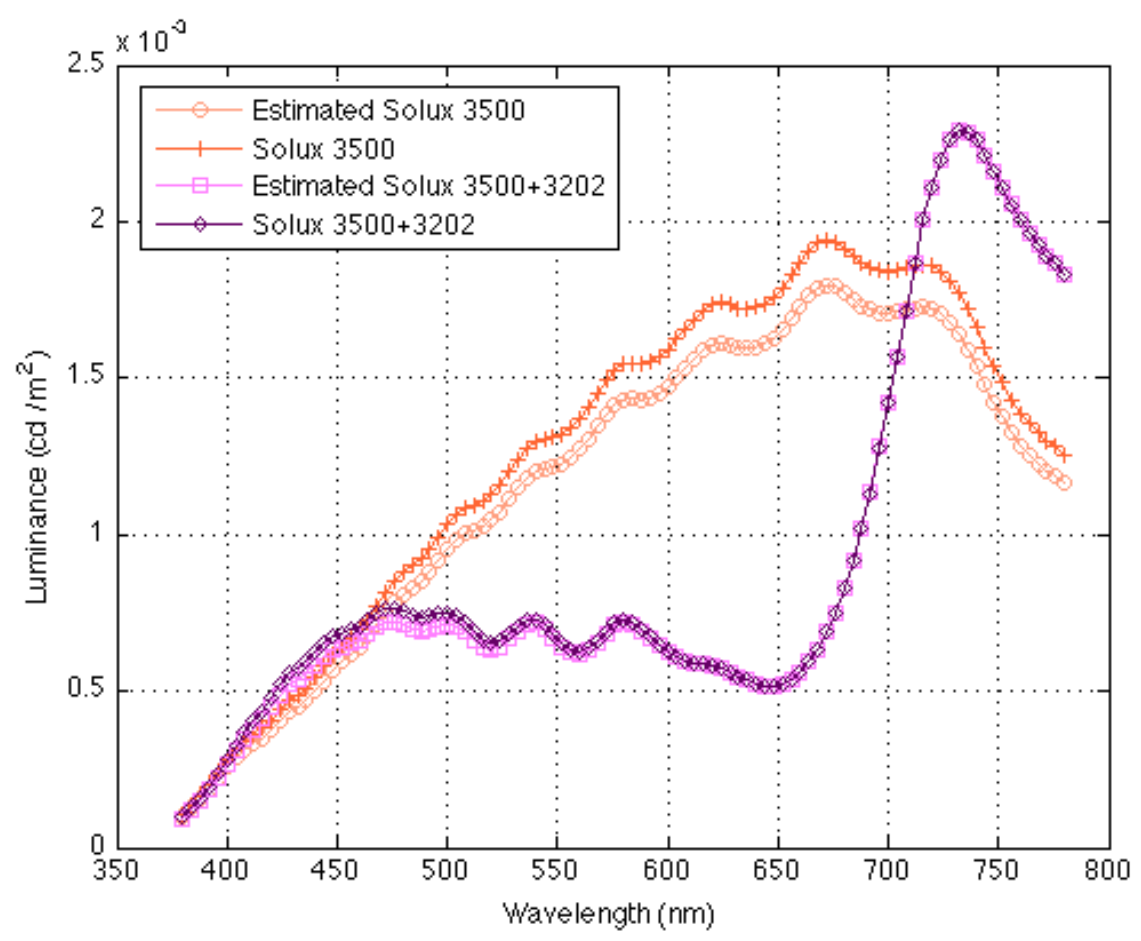

(f) Estimated vs. Original Solux $3500 \% 3500+3202$ Illuminant

Figure 4.5 Comparing the estimated power spectrum of eleven different illuminants with their relative real power spectra measured by a spectrometer.

Additional images are shown in Figure 4.6. These images were downloaded from the Internet and showed some lossy-compression artifacts. We assumed that the gamma used for image display correction was 2.2. Despite this source of noise, our method efficiently separated and rendered the specular reflectance components on both images.

We implemented our algorithm on an Intel ${ }^{\mathrm{TM}}$ Core 2 Duo processor, having a clock speed of $2.53 \mathrm{GHz}$. The performance registered was performed based on a CPU implementation. For images of size $100 \times 100$ the rendering process took an average of 1.01 seconds, for $300 \times 300$ images took an average of 9.87 seconds, for images of size 500x500 the average processing time was 31.01 seconds, and images of size 1000x1000 it took 112.49 seconds. 
We also evaluated the duration of the reflectance decomposition step and found it to take $38 \%$ of the total simulation time for images of size $1000 \times 1000$, while the specular reflection-modeling step consumed $61 \%$ of the total computing time.

\begin{tabular}{|c|c|c|}
\hline Illuminants & Spectral Est. Error (\%) & $\begin{array}{c}\text { Chromaticity Mean Error } \\
\text { (\%) }\end{array}$ \\
\hline \hline Philips-ulm & 4.593 & 0.067 \\
\hline Solux 3500+3202 & 2.373 & 0.276 \\
\hline Solux 3500 & 7.474 & 0.582 \\
\hline Solux 4100+3202 & 4.594 & 0.331 \\
\hline Solux 4100 & 6.412 & 0.352 \\
\hline Solux 4700+3202 & 5.639 & 0.571 \\
\hline Solux 4700 & 5.714 & 0.218 \\
\hline Syl_50MR16Q+3202 & 3.382 & 0.238 \\
\hline Syl_50MR16Q & 7.494 & 0.514 \\
\hline Syl_CWF & 3.028 & 0.3 \\
\hline Syl_WWF & 4.227 & 0.139 \\
\hline
\end{tabular}

Table 4.1 Illumination mean errors for all illuminant sources using the spectral evaluation method (middle) and image chromaticity method (right).

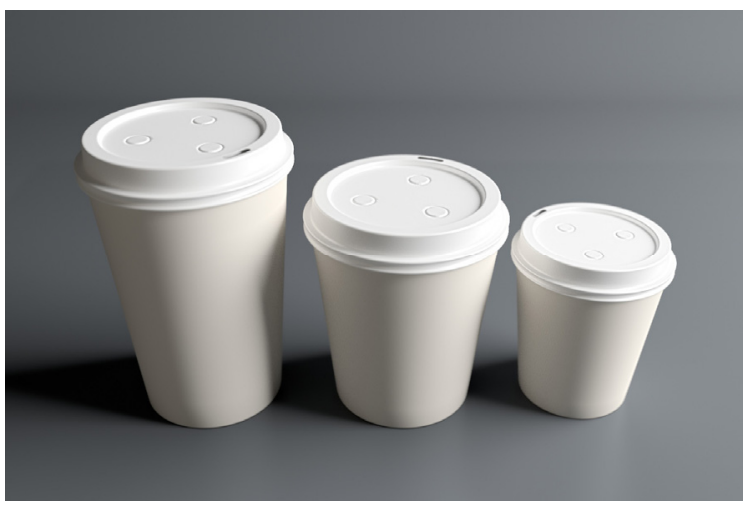

(a) Input Image

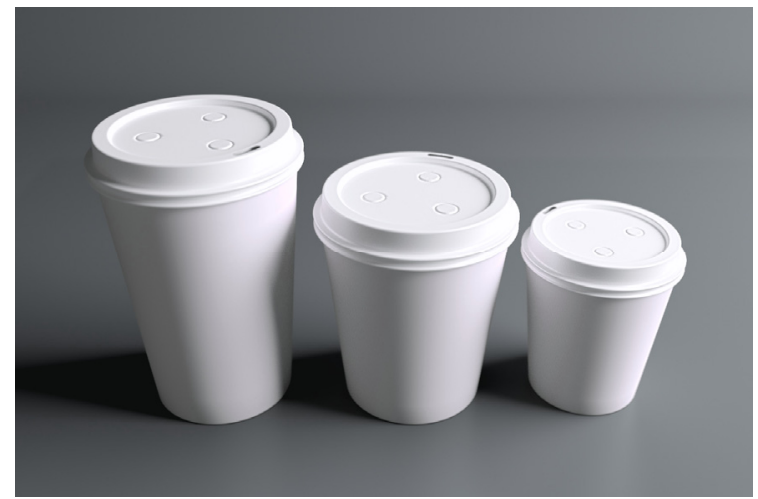

(b) White light rendering $(0.1,0.1,0.15)$

Figure 4.6 Example of rendering scenes with uniform illumination distribution using our proposed method with the shown thresholds. 


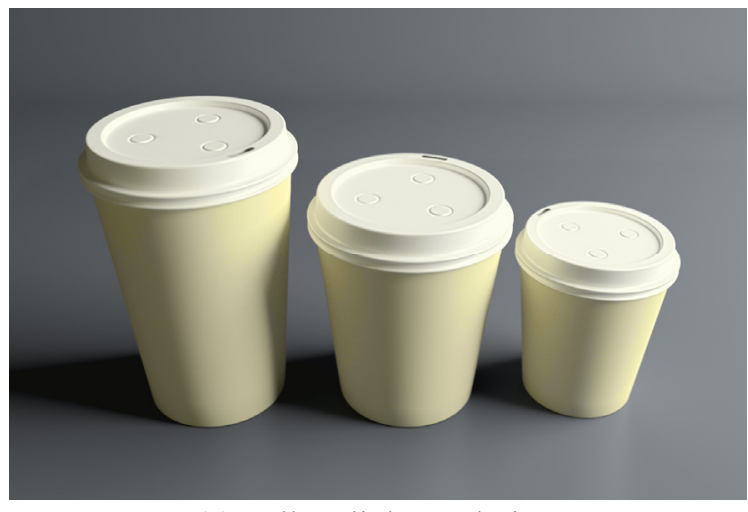

(c) Yellow light rendering

$(1.0,1.0,2.9)$

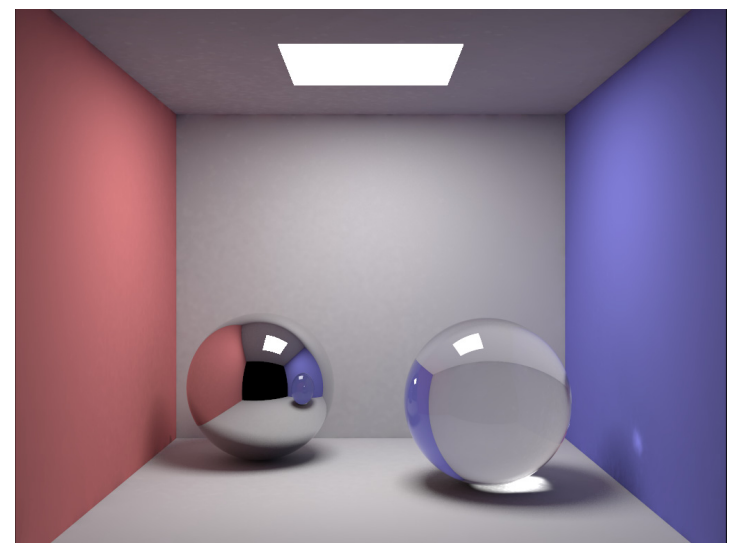

(e) Input Image

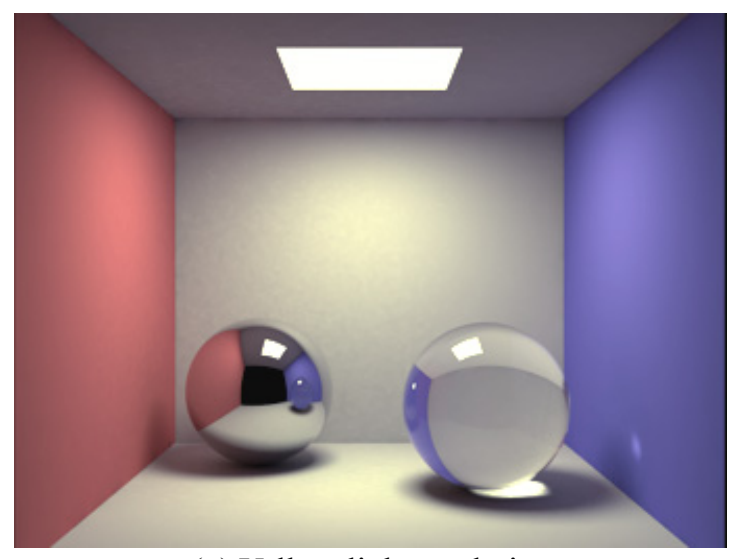

(g) Yellow light rendering

$(1.12,1.1,1.0)$

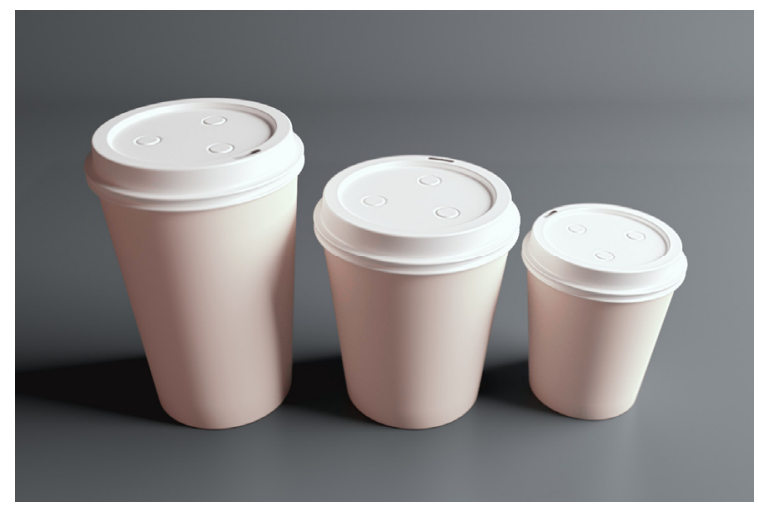

(d) Red light rendering

$(7.9,1.0,1.0)$

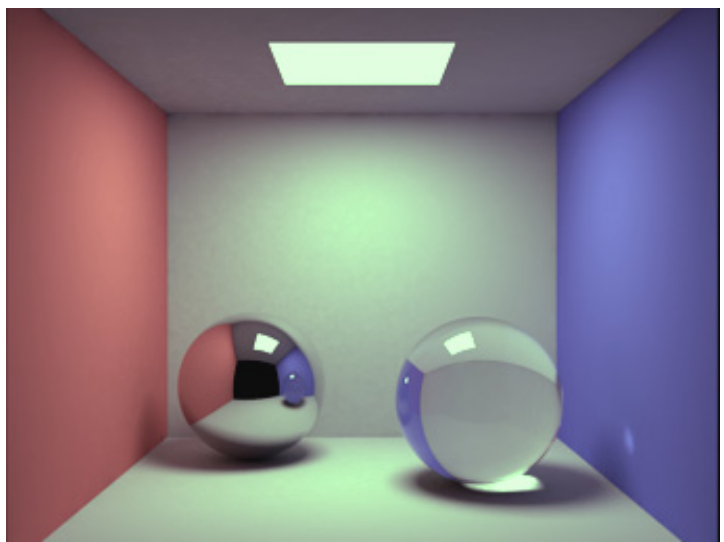

(f) Green light rendering $(0.95,1.1,0.95)$

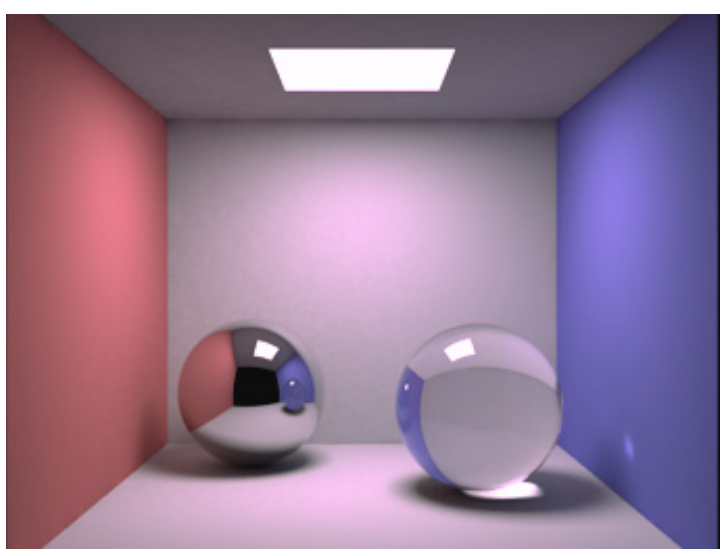

(h) Tungsten light rendering $(1.2,1.0,1.11)$

Figure 4.6. (Cont.) Example of rendering scenes with uniform illumination distribution using our proposed method with the shown thresholds. 


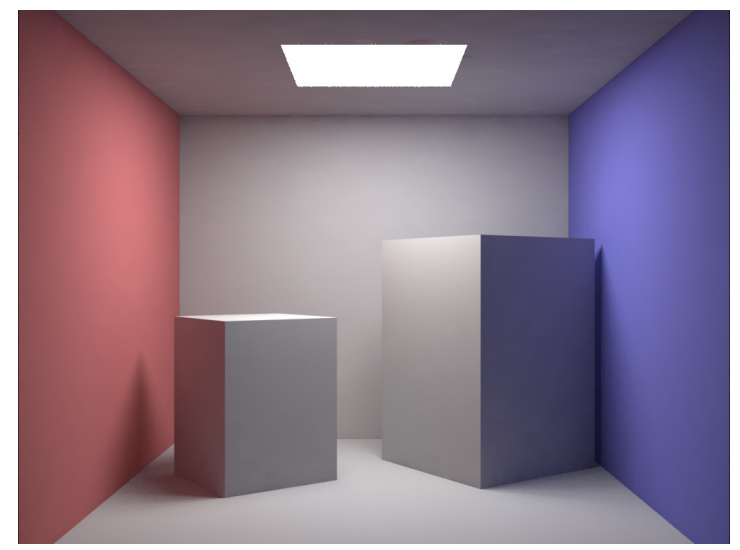

(a) Input image

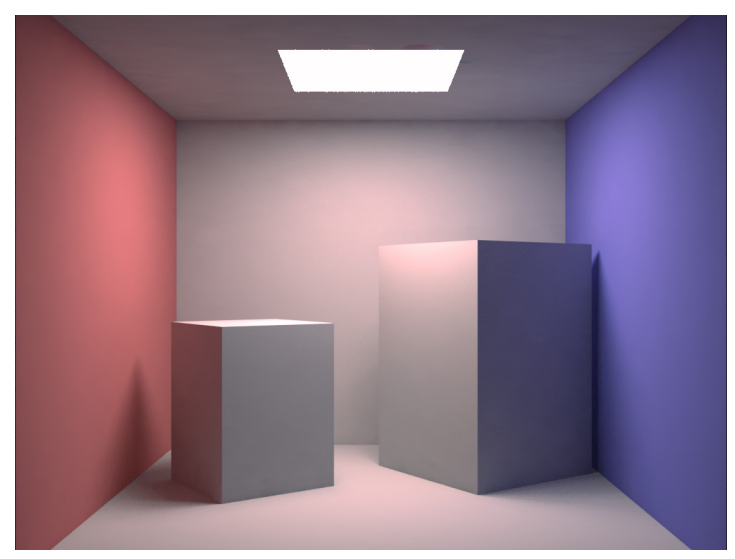

(b) Rendered image

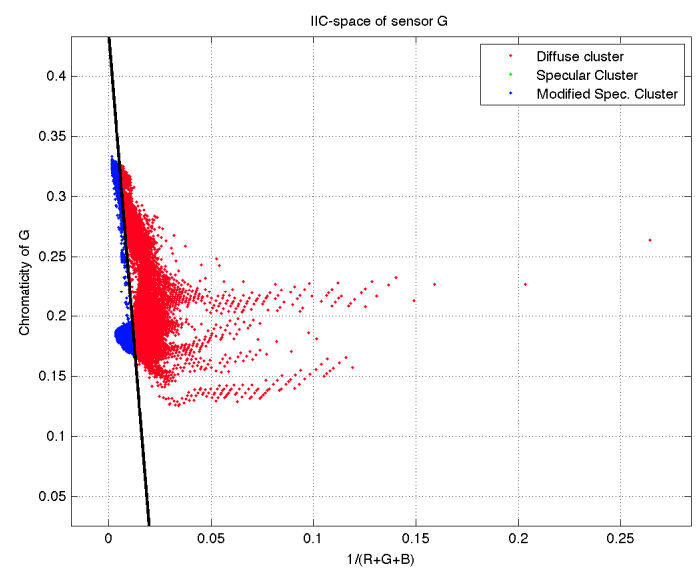

(d) Green channel

$$
\eta_{G}=1
$$

Figure 4.7 Illustration of the presented decomposition method into specular components (green) and diffuse components (red) overlapped by the modeling process (blue). (a) Input image. (b) The result of rendering (a). (c) Reflectance separation and modeling over the red channel. (d) Reflectance separation and modeling over the green channel. (e) Reflectance separation and modeling over the blue channel.

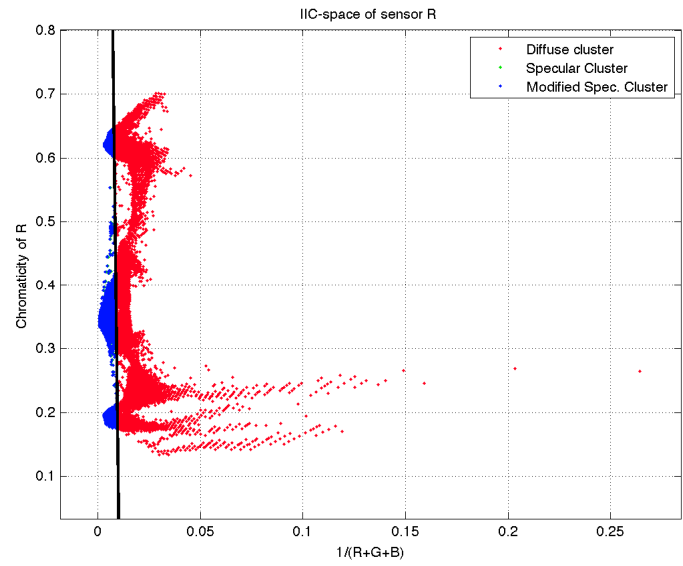

(c) Red channel $\eta_{R}=1.1$

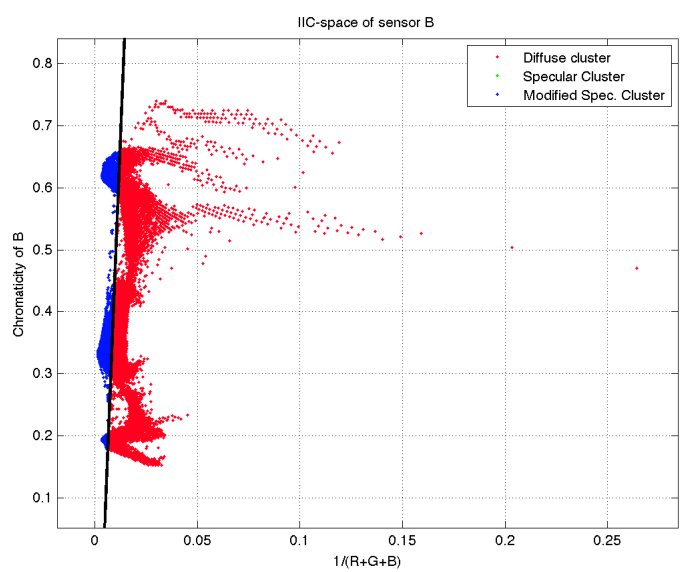

(e) Blue channel $\eta_{B}=1$ 


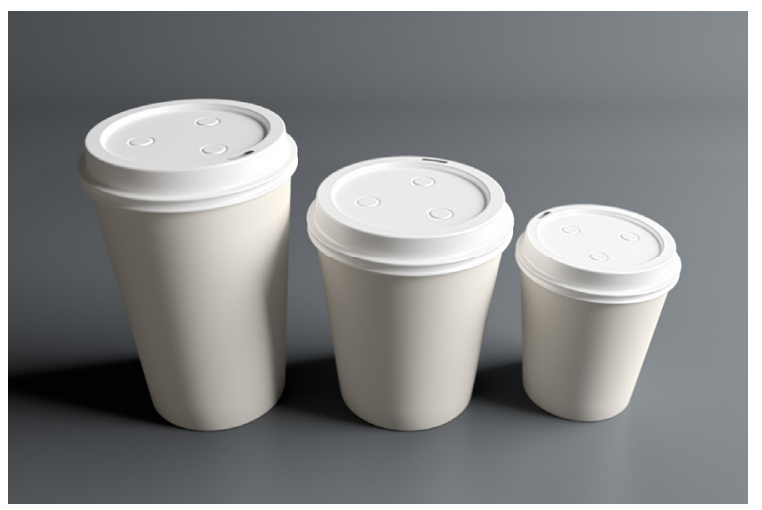

Figure 4.8 Illustration of the presented decomposition method into specular components (green) and diffuse components (red) overlapped by the modeling process (blue). (a) Input image. (b) The result of rendering (a). (c) Reflectance separation and modeling over the red channel. (d) Reflectance separation and modeling over the green channel. (e) Reflectance separation and modeling over the blue channel.

(a) Input image
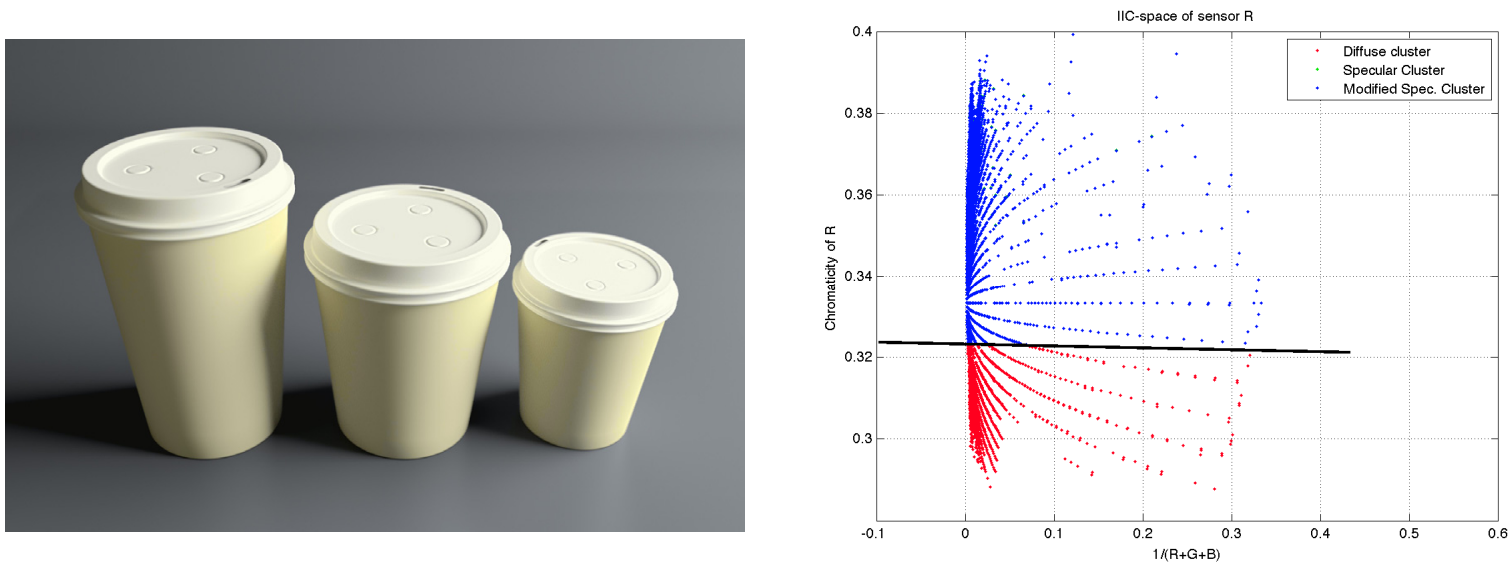

(b) Rendered image

(c) Red channel

$$
\eta_{R}=1.0
$$

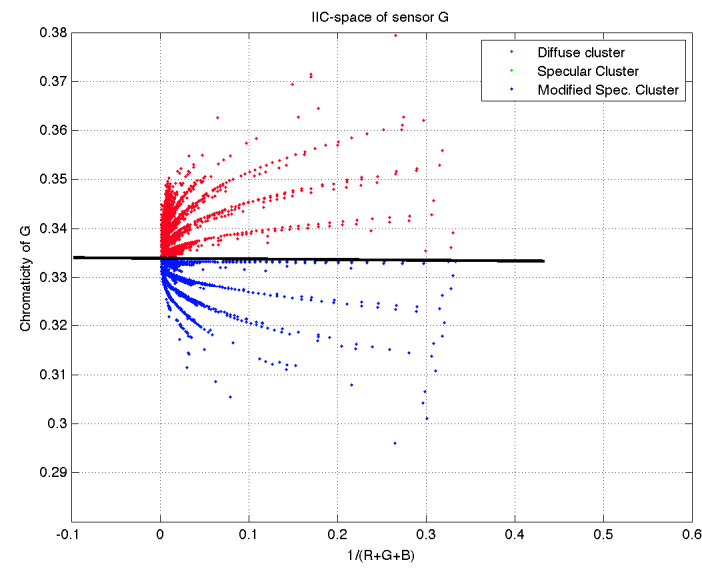

(d) Green channel

$$
\eta_{G}=1.0
$$

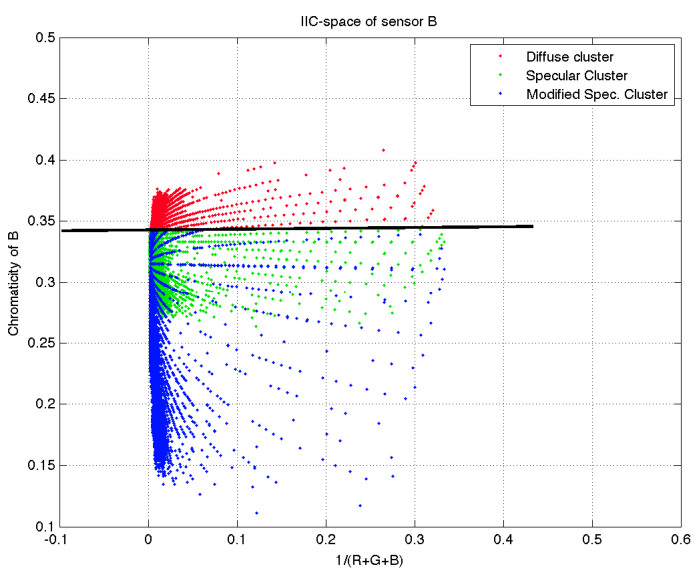

(e) Blue channel

$\eta_{B}=2.9$ 


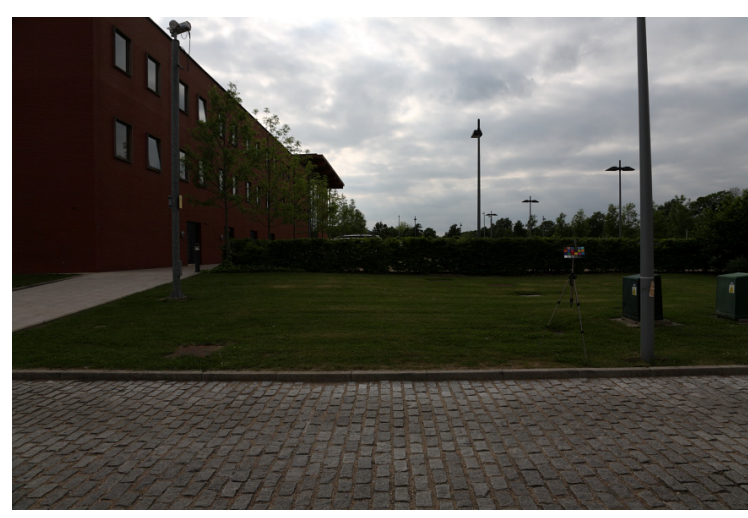

(a) Input image

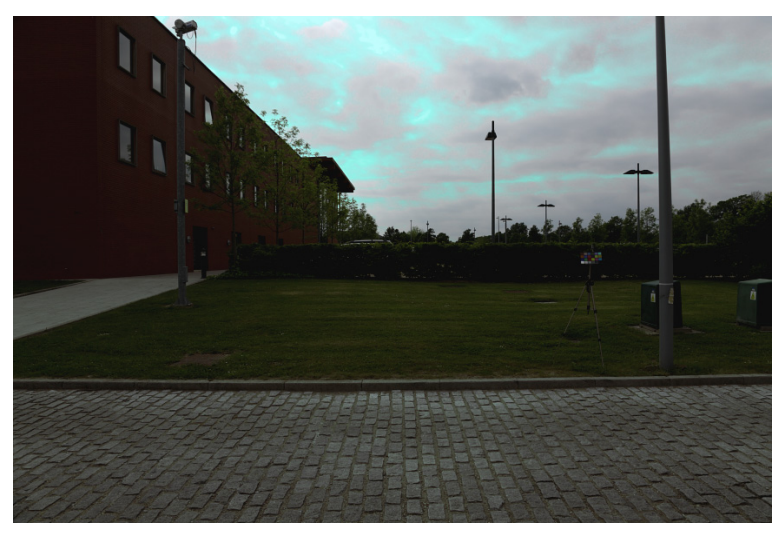

(b) Rendered image

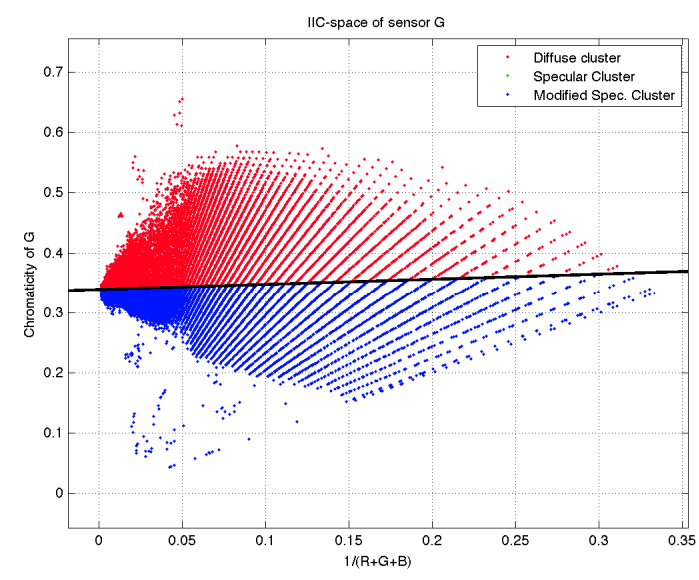

(d) Green channel

$\eta_{G}=1.0$
Figure 4.9 Illustration of the presented decomposition method into specular components (green) and diffuse components (red) overlapped by the modeling process (blue). (a) Input image. (b) The result of rendering (a). (c) Reflectance separation and modeling over the red channel. (d) Reflectance separation and modeling over the green channel. (e) Reflectance separation and modeling over the blue channel.

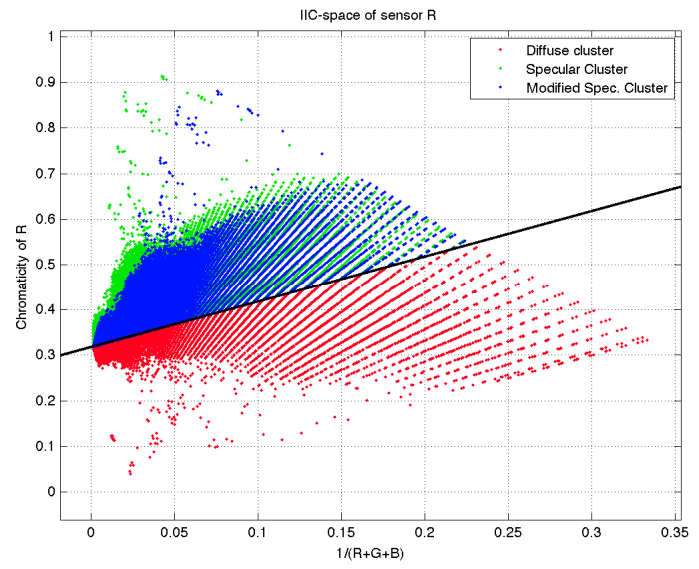

(c) Red channel $\eta_{R}=0.88$

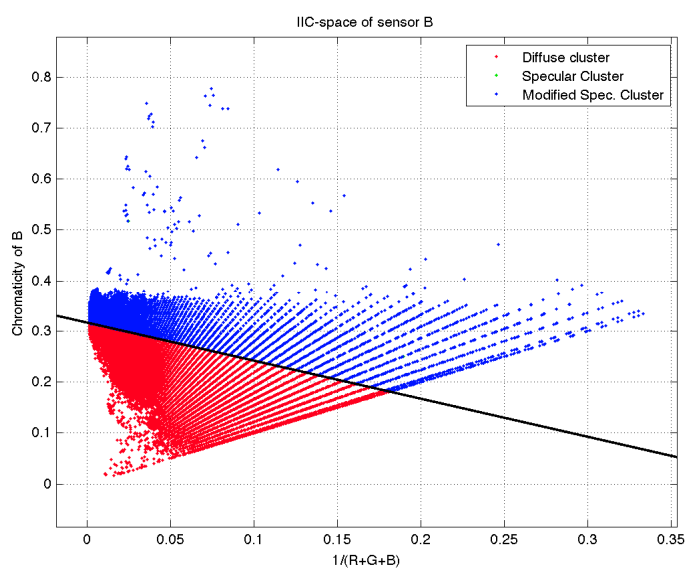

(e) Blue channel $\eta_{B}=1.0$ 


\section{CHAPTER V}

Color constancy based dichromatic objects reflectance decomposition

\subsection{Introduction}

The human visual system possesses a powerful capability in determining objects' surface color properly despite large changes in scene illumination. Our color perception takes place by estimating the actual object's surface spectral reflectance regardless of the light color affecting the object's illumination reflectance. This visual ability in determining the true surface color appearance of objects is called color constancy. Computer vision systems, on the other hand, still can't accomplish this task without prone errors. Thus, finding methods that can recover the actual color of object surfaces correctly can benefit many applications for image retrieval, object rendering, color-based object recognition, reflectance estimation, etc.

Many color constancy methods have been proposed in literature and can be categorized into two separate groups, statistics-based approaches and physics-based approaches. The first group consists of algorithms that correlate information acquired in a learning phase with possible information related to the image features such as light sources or reflectance distributions of objects in natural image scenes. Gamut mapping algorithm [Forsyth, 1990] is one of the first methods to use this kind of approach. This algorithm assumes for a given illuminant only limited number of colors can be observed. The set of possible color values for canonical illuminant, generally a white illuminant, is called the canonical gamut. The gamut mapping method establishes a linear map from a pre-learned distribution of colors (canonical gamut) to the color distribution of a given image. Other 
derivations of the gamut mapping approach have been proposed such as the Gamutconstrained illuminant estimation [Finlayson, 2006], Color-by-Correlation [Finlayson, 2001], and derivative-based gamut mapping [Gijsenij, 2010]. Other statistics-based approaches employ Bayes's rule to estimate the illumination distribution using standard methods such as minimum-mean-squared error (MMSE), maximum-local-mass estimation (MLM), or maximum-a-posteriori (MAP) method [Brainard, 1997][Brainard, 2006]. For these methods, the illuminant spectra distribution and the surface reflectance distribution are linearly modeled with a Gaussian basis. For instance, the color-bycorrelation method [Barnard, 2000][Brainard, 1997] characterizes all plausible observed image colors by finding a correlation matrix of a set of known illuminants.

Furthermore, simpler approaches that assume spatial statistics of image colors can be related to the Grey-world hypothesis [Brooks, 1985], which assumes that the surface reflectance spatial average in a scene is achromatic, the Grey-edge hypothesis [van de Weijer, 2005], which similarly assumes that the average edge in a scene image is also achromatic, and the spatial correlation approach introduced by Chakrabarti et al. [Chakrabarti, 2010], which estimates the illuminant color by measuring the spatial correlations that exists between image points.

Different from the methods based on the first approach, physics-based color constancy approaches analyze the physical process of the light reflected from surfaces. Understanding this process in which light interacts with the surface reflectance was the objective of many scientists. Based on the surface reflectance of the input image, physicsbased methods can be derived by assuming that the image surface reflectance is either completely diffuse, or follow the dichromatic model introduced by [Shafer, 1985]. Based 
on Shafer's model, the dichromatic color constancy methods solve the illumination estimation problem by analyzing the surface reflectance and estimating the color of the specular reflections emitted from object surfaces. Lee's method in estimating the illumination reflectance [Lee, 1986] uses image highlights of at least two surface colors. The illumination reflectance is estimated by finding an intersection of two or more dichromatic lines in the chromaticity space. However his method requires a heavy segmentation of the surface color representing the reflection highlights as a preprocessing step. Similar to Lee's methods, Lehman and Palm [Lehmann, 2001] developed a technique that assumes a uniform color surface in each image highlight region.

The proposed methods performs well on restricted images with uniform color surfaces however, highly textured surfaces show some inaccuracy in estimating the illumination chromaticity. Finlayson and Schaefer [Finlayson, 2001] found that the chromaticities of common light sources all follow closely to Planckian-locus of blackbody radiators. They were able to estimate the chromaticity of the illuminant simply by intersecting the dichromatic line with the Planckian locus. Recently, Tan et al. [Tan, 2004] obtained a direct correlation between the illumination chromaticity and image chromaticity by analyzing image highlight regions. Using this relation, they estimated illumination chromaticity by counting the number of intersections image chromaticity lines projected on the Hough space for both uniform and non-uniform surfaces.

In this chapter we estimate illumination chromaticity in images based on the dichromatic reflectance approach. By projecting image reflectance into the inverse-intensity chromaticity space, we select image highlights by separating the diffuse and illumination reflectance distributions using the Mean-shift-decomposition technique presented in 
chapter III. These highlights are then evaluated to sift only the prominent surface reflectance that reflects the exact illumination chromaticity using histogram analysis. The resulted surface reflectance are then again assessed using the $M S D$, and the illumination chromaticity is estimated by intersecting the first and the second orthogonal basis of the newly calculated covariance matrix. Our method has the advantage of not using any preprocessing color segmentation technique to isolate the reflectance highlight.

\subsection{Contributions}

The illumination chromaticity of each image color channel is estimated using the following considerations:

- By assuming a dichromatic model, the illumination highlights reflecting the illumination chromaticity are obtained using the Mean-shift-decomposition methods introduced in chapter-III.

- A voting-scheme that assumes a small set of surface reflectance is used to characterize the most prominent image pixels that reflects the illumination chromaticity color based on histogram analysis.

- The illumination chromaticity is estimated by projecting the intersection of the first and second orthogonal bases onto the image chromaticity axis of the IICspace.

- We assess our method on a large dataset comprising real-world natural images. 


\subsection{Overview}

Section 5.4 presents a literature review of the main methods used for color constancy. Section 5.5 discusses the model used as a basis in the method presented in this chapter. Section 5.6 describes the mapping model used to generate canonical images corrected by the method introduced in this chapter. Section 5.7 proposes a voting scheme method used for selecting the prominent specular reflectance component. Section 5.8 presents the illumination chromaticity estimation in an image. In section 5.9, we evaluate our proposed method using real world images. Section 5.10 concludes this chapter with a summary of the proposed color constancy method.

\subsection{Dichromatic Reflectance Model}

The combination of matte reflectance with a geometry dependent highlight component is well defined by the dichromatic reflectance model described in section 3.5. In real world, based on their surface properties, most objects can be categorized into two groups: homogeneous and inhomogeneous objects. Homogenous objects such as glass, metals, and crystals have a uniform refractive index through their surface and produce mainly specular reflectance. However, inhomogeneous objects such as ceramics, acrylics, and plastics have varying refractive indices in their surface and consequently this kind of objects present both diffuse and specular reflectance. Color constancy methods based on the dichromatic reflectance model estimate the color of the light source by analyzing specular objects' highlights. As seen in chapter III, for surfaces that are well-represented by the dichromatic reflectance model, the spectral reflectance distribution of the specular 
component is assumed to be similar to the spectral energy distribution of the incident light, while the spectral reflectance distribution of the diffuse component is assumed to depend on the material properties describing the object surface. This assumption termed as the neutral interface reflection assumption is adopted by many color constancy methods based on the dichromatic reflectance model. Similarly, we ground our work on this assumption to estimate the illumination color chromaticity.

\subsection{Diagonal Model Transformation}

Color constancy is defined as correcting an image taken under an unknown light source so it appears to be taken under a canonical light source. This chromatic transformation can be considered as a module of the Chromatic Adaptation Transform (CAT) [Fairchild, 2005]. A first step will be to estimate the color of the illumination source, followed by a transformation of the original image [Hordley, 2006]. This color adaptation is modeled using a linear transformation based on the von Kries chromatic model, which states that chromatic adaptation is an independent gain regulation of the three sensors in the human visual system. By assuming a narrowband sensor sensitivity, in this chapter we use the von Kries chromatic model [von Kries, 1970], also known as the diagonal model, to transform all colors of the input image taken under an unknown light source to colors as they appear under a canonical light source without applying any spectral sharpening methods [Finlayson, 1994] or changing the color basis [Chong, 2007]. The diagonal model used is defined by the following expression:

$$
I_{k, t}=D_{u, t} I_{k, u}
$$

where $k=\{r, g, b\}$ is the input image color channel, $I_{u}$ is the input image taken under an 
unknown spectral illuminant $u, I_{t}$ is the transformed input image using the estimated canonical illuminant $c$, and $D_{u, t}$ is a diagonal matrix which maps the input image $I_{u}$ to the transformed image $I_{t}$ as follows:

$$
\left[\begin{array}{c}
I_{r, c} \\
I_{g, c} \\
I_{b, c}
\end{array}\right]=\left[\begin{array}{ccc}
d_{1} & 0 & 0 \\
0 & d_{2} & 0 \\
0 & 0 & d_{3}
\end{array}\right]\left[\begin{array}{c}
I_{r, t} \\
I_{g, t} \\
I_{b, t}
\end{array}\right]
$$

This model approximates well the illuminant changes and has been adapted by several color constancy methods [West, 1982] [Finlayson, 1994] [Funt, 2000] [Tan, 2004], [Kawakami, 2005] [Gijsenij, 2011].

\subsection{Surface Reflection Selection}

The MSD method decomposes image reflectance components projected on the IIC-space into two separate reflectance sets representing the diffuse and specular reflectance distributions. In this section, we retrieve the most prominent surface reflectance points from the set of specular reflectance components obtained from the MSD decomposition technique to define the illumination chromaticity. Omer and Werman [Omer, 2004] noticed that natural scenes images are dominated by a small set of surface color and applied this assumption to applications targeting object segmentation and image compression. Similarly, we use this hypothesis to extract a small set of surface reflectance components that delineates the image illumination chromaticity.

We begin by constructing a histogram $H_{k}$ representing the specular reflectance distribution of each image chromaticity channel. The histogram tells us how many specular components have a particular chromaticity intensity for a given color channel $k$. 
Let $n_{b}$ denotes the number of buckets in the histogram and $H_{k}(j)$ the number of specular reflectance components in each color channel $k$ that have intensity $j$. Instead of choosing the reflectance component with the maximum intensity [Finlayson, 2002], we find the local maxima intensities in the histogram. We process the specular reflectance components starting with the highest local maxima intensity value, for a given reflectance component $\boldsymbol{x}_{i}$, where $i=1,2, \ldots, N$, from the specular reflectance distribution projected on the reflectance histogram, if the value of $\boldsymbol{x}_{i}$ fall within the histogram bucket interval satisfying:

$$
H_{k}\left(j_{\max }\right)-H_{k}\left(j_{\max }+\delta\right) \leq 0 \quad \text { and } \quad H_{k}\left(j_{\max }\right)-H_{k}\left(j_{\max }-\delta\right) \geq 0
$$

where $j_{\max }$ is the intensity of the histogram buckets representing the local maxima values, and $\delta$ is a rational threshold defining the number of selected histogram buckets $n_{b}$, we say that the specular reflectance $\boldsymbol{x}_{i}$ is selected to represent the specular points for illumination chromaticity estimation. The reflectance components that were selected satisfying Equation 5.3 are marked and the remaining unmarked reflectance elements are then used for a second turn voting round to depict the second most popular reflectance components. This process continues along all local maxima intensities to form a smaller set of specular reflectance distribution $\tilde{S}_{k}$. This technique works well for many typical images, however in certain situations, particularly outdoor scenes, the presence of noise in the surface reflectance distribution is inevitable. In these cases, instead of processing the specular reflectance components starting with the highest local maxima value it is practical to begin with the local maxima intensities that assemble the most specular reflectance components within the threshold $\delta$. This ensure that the new specular 
reflectance distribution $\tilde{S}_{k}$ contains values that do not take account the highest specular reflectance intensities.

\subsection{Illumination Chromaticity Estimation}

In the previous section we described a method to derive a set of surface reflectance points that govern the scene illumination reflectance distribution, in this section we estimate the illumination chromaticity by analyzing the changes influencing the spread of the derived specular reflectance set $\tilde{S}_{k}$. We make use of the $M S D$ method presented earlier to find the correlation attributes associated in $\tilde{S}_{k}$. By finding the first and second orthogonal bases of the reflectance set $\tilde{S}_{k}$ using Equation 3.6, the equations of the lines describing the variance between each specular reflectance component are expressed as follows:

$$
\begin{aligned}
& f_{1, k}(n)=\tilde{v}_{1, k}\left(\frac{1}{\sum_{j \in k} \rho_{j}(n)}-u_{1, k}\right)+u_{2, k} \\
& f_{2, k}(n)=\tilde{v}_{2, k}\left(\frac{1}{\sum_{j \in k} \rho_{j}(n)}-u_{1, k}\right)+u_{2, k}
\end{aligned}
$$

where $f_{1, k}$ and $f_{2, k}$ are the line equations representing the first and second orthogonal basis respectively, the gradients $\tilde{v}_{1, k}$ and $\tilde{v}_{2, k}$ depict the direction of the first and second eigenvector of the orthonormal basis vector $\boldsymbol{U}$ corresponding to the first and second highest eigenvalue in $\Lambda$, and $u_{1, k}$ and $u_{2, k}$ are the means of the projected reflectance 
components set $\tilde{S}_{k}$ on the IIC-space. Then, by projecting the point intersecting both basis lines on the IIC-space chromaticity axis, the illumination chromaticity of each color channel is determined as follows:

$$
\Psi_{k}=f_{1, k}(n) \cap f_{2, k}(n)
$$

\subsection{Experimental Results}

We assess the performance of the proposed color constancy method on two different datasets of colorful images. One contains images registered under controlled indoor illumination [Barnard, 2002], and the other contains images of outdoor scenes registered under real world conditions [Ciurea, 2004]. The first dataset consists of images taken under eleven uniform illuminant sources simulating eight different daylight spectra and three different fluorescent spectra. The second dataset contains approximately 11,000 images registered under an ambient illuminant having a neutral grey sphere attached to the camera. This sphere is used as a ground-truth to estimate the illuminant color in the scene. The goal of our experiment is to determine the accuracy of the retrieved scene illuminant. For all images in the datasets, we measure how close the estimated illuminant is equivalent to the actual color of the scene using the angular error evaluation:

$$
\mathcal{E}=\cos ^{-1}\left(\frac{e_{g} \cdot e_{e}}{\left\|e_{g}\right\| \cdot\left\|e_{e}\right\|}\right)
$$


where $e_{g}$ and $e_{e}$ are the color of the light source and the color of the estimated illumination respectively, and $\|$.$\| denotes the Euclidean norm.$

The following figure compares the result of using the proposed color constancy method with other proposed method in the literature including Shade of Grey [Finlayson, 2004], Grey Edge [van de Weijer, 2005], and Grey World [Brooks, 1985].

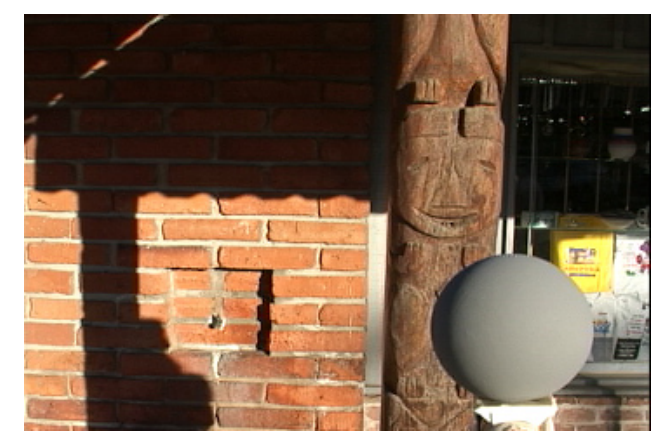

Input image

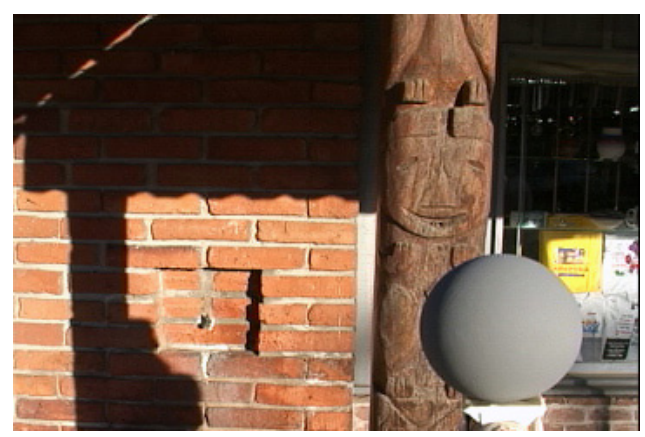

Proposed method $\left(1.38^{\circ}\right)$

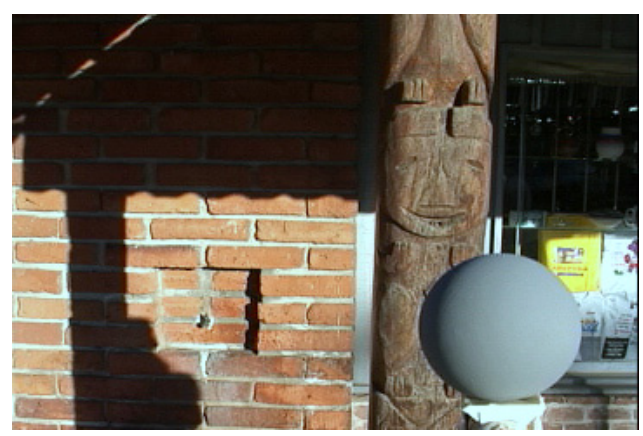

Grey Edge $\left(5.16^{\circ}\right)$

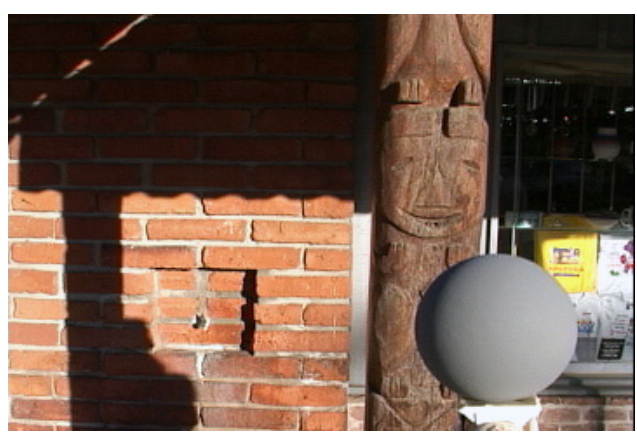

Ideal correction

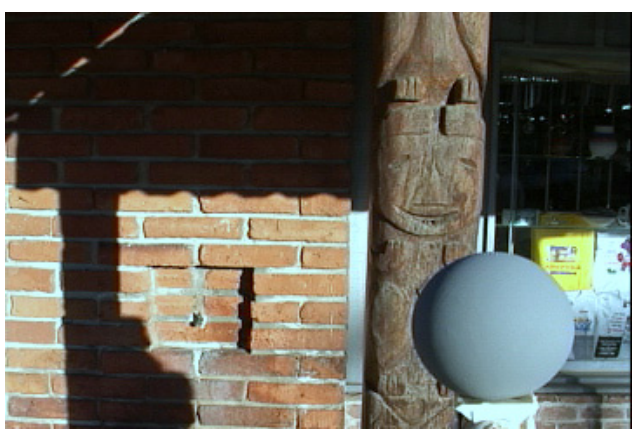

Shade of grey $\left(7.55^{\circ}\right)$

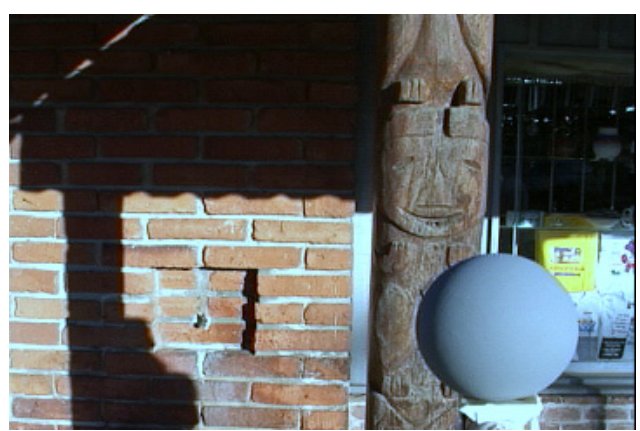

Grey World $\left(11.13^{\circ}\right)$

Figure 5.1 The results of the proposed algorithm compared to other color constancy methods on the realworld dataset. The illumination angular error is shown under each image. 


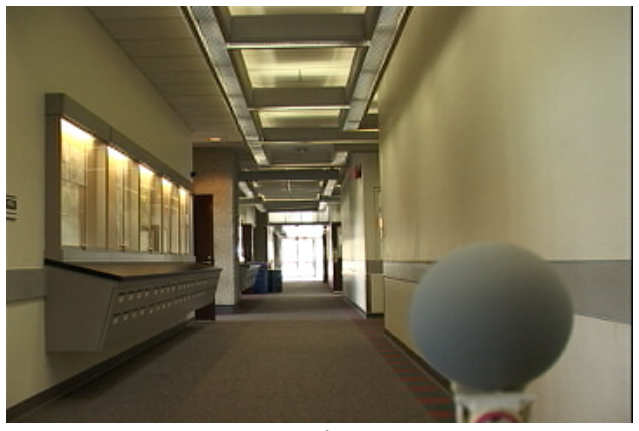

Input image

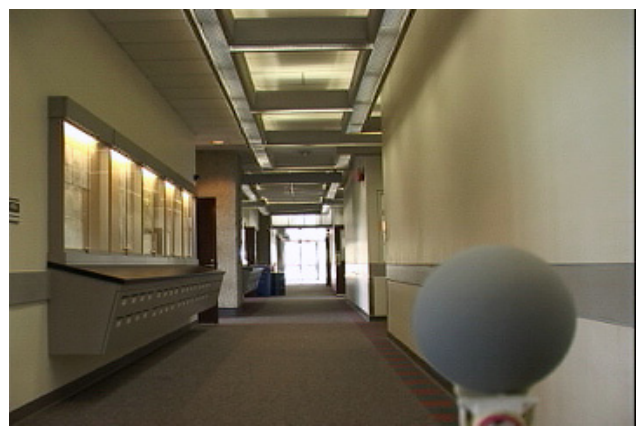

Proposed method $\left(2.32^{\circ}\right)$

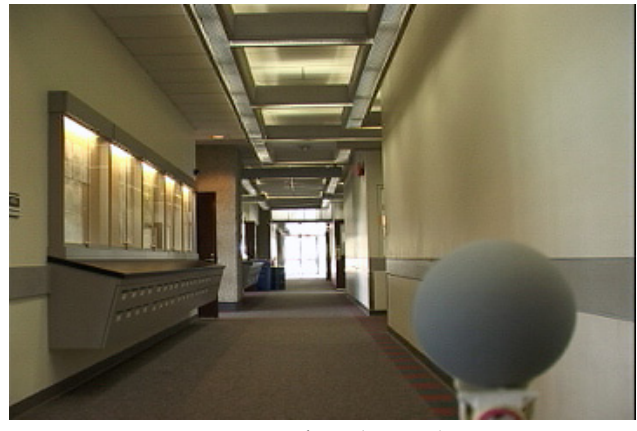

Grey Edge $\left(2.85^{\circ}\right)$

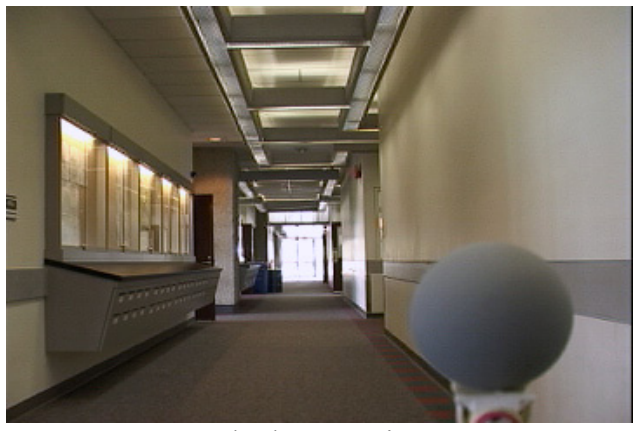

Ideal correction

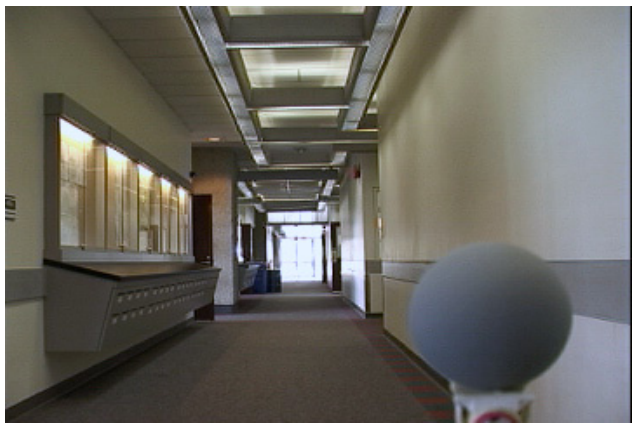

Shade of grey $\left(3.03^{\circ}\right)$

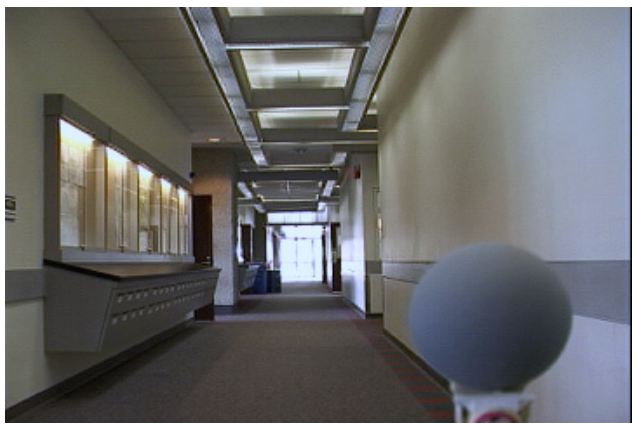

Grey World $\left(5.25^{\circ}\right)$

Figure 5.2 The results of the proposed algorithm compared to other color constancy methods on the realworld dataset. The illumination angular error is shown under each image. 


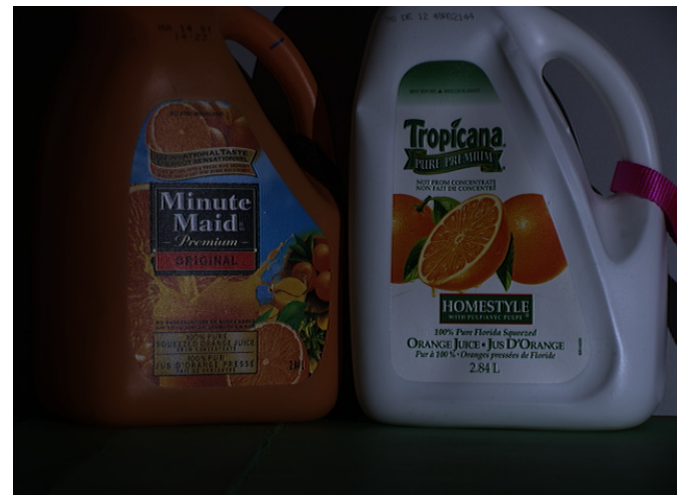

Input image

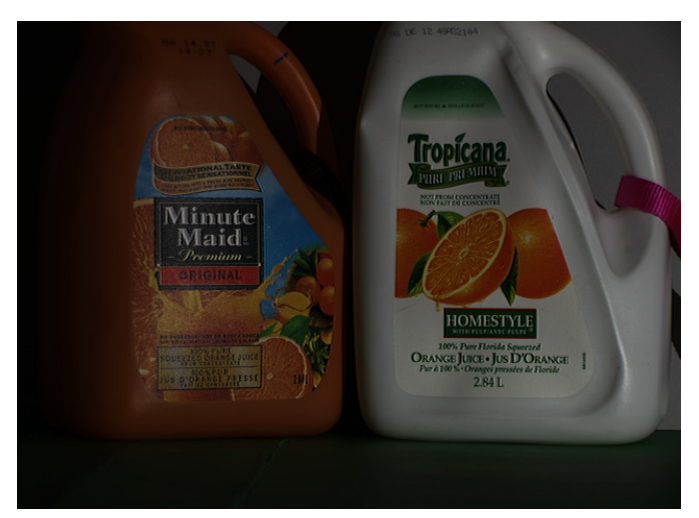

Proposed method $\left(0.21^{\circ}\right)$

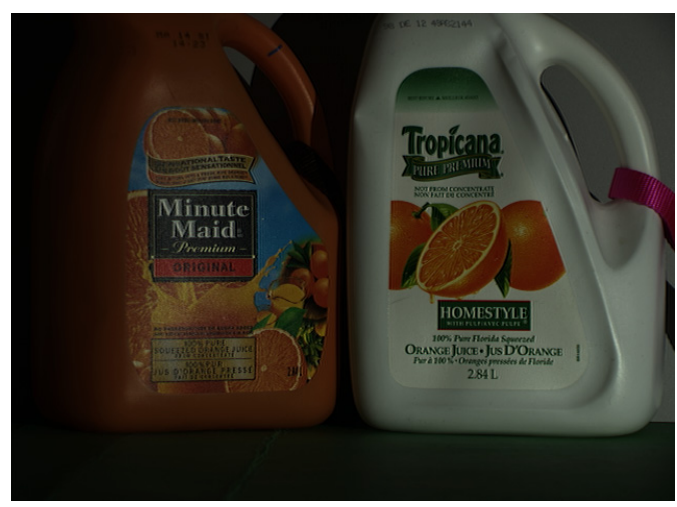

Grey Edge $\left(2.12^{\circ}\right)$

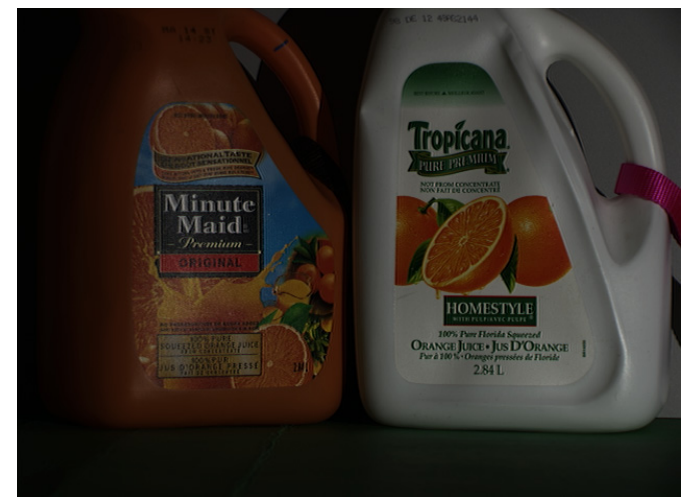

Ideal correction

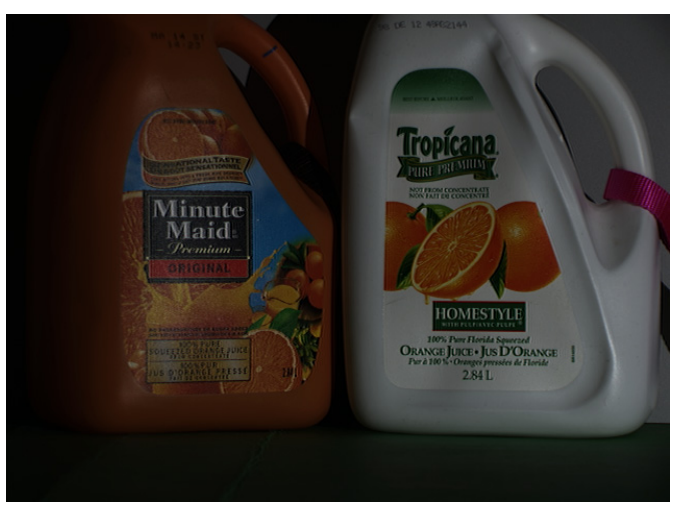

Shade of grey $\left(1.63^{\circ}\right)$

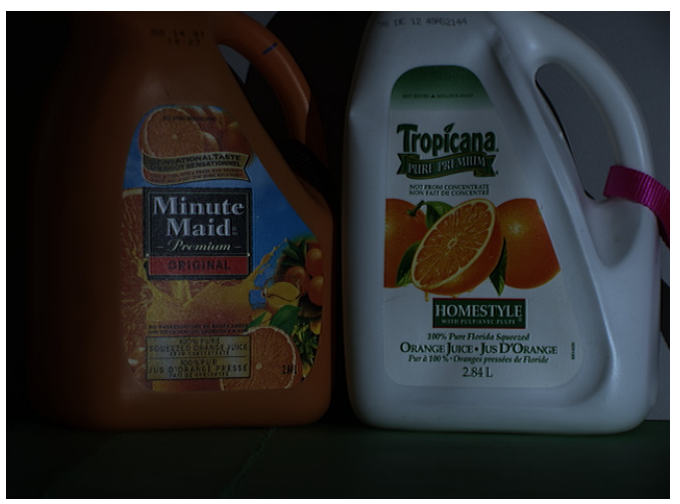

Grey World $\left(8.53^{\circ}\right)$

Figure 5.3 The results of the proposed algorithm compared to other color constancy methods on the controlled indoor illumination dataset. The illumination angular error is shown under each image 


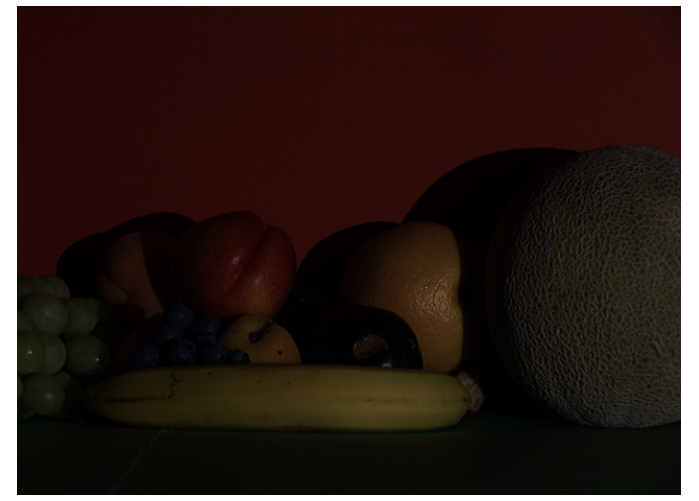

Input image

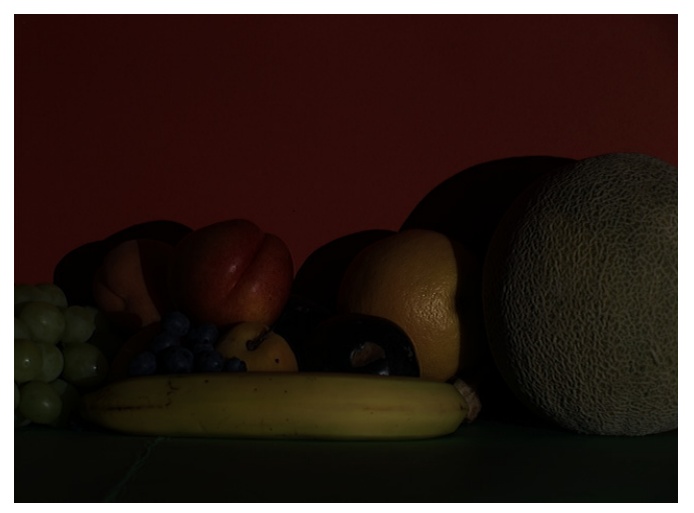

Proposed method $\left(5.72^{\circ}\right)$

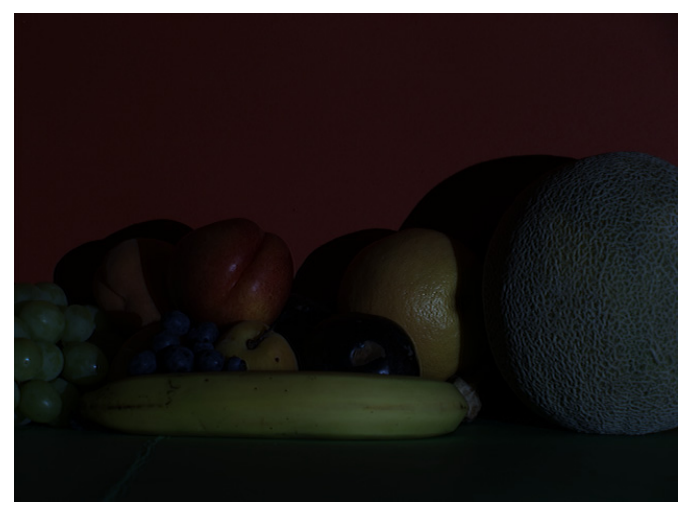

Grey Edge $\left(23.65^{\circ}\right)$

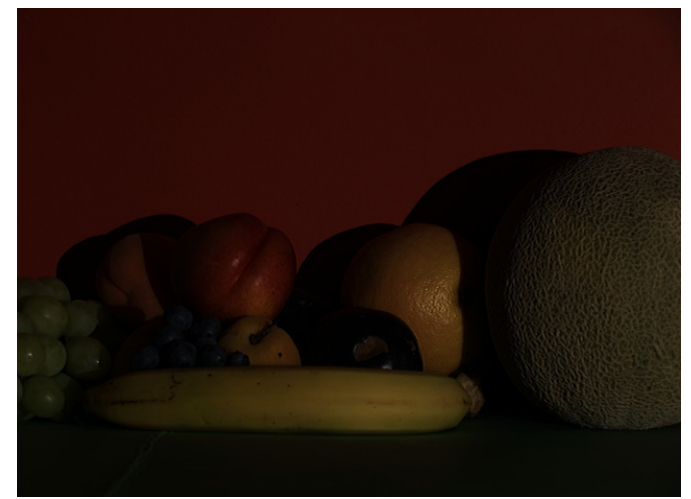

Ideal correction

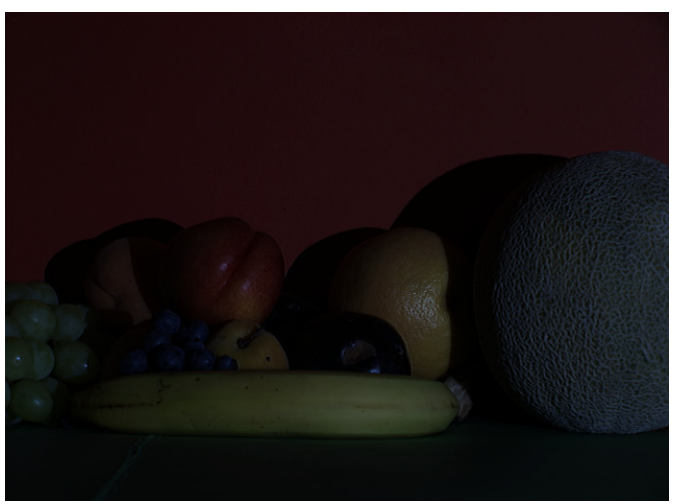

Shade of grey $\left(24.28^{\circ}\right)$

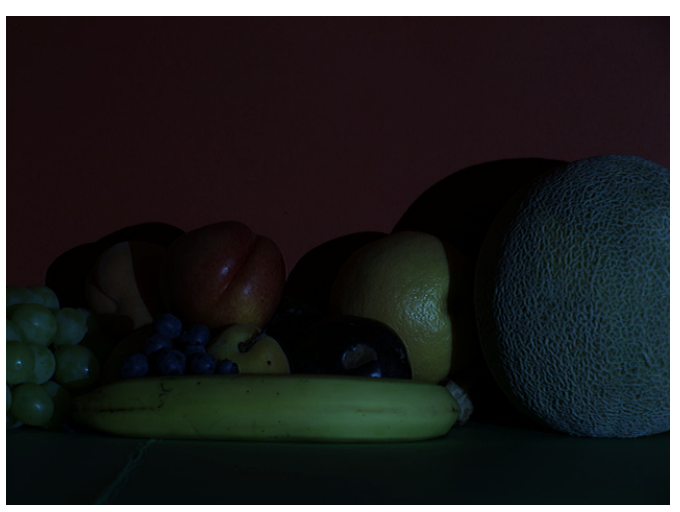

Grey World $\left(33.11^{\circ}\right)$

Figure 5.4 The results of the proposed algorithm compared to other color constancy methods on the controlled indoor illumination dataset. The illumination angular error is shown under each image. 


\section{CHAPTER VI}

\section{Conclusion}

This dissertation established a new mathematical framework for the separation of specular and diffuse reflectance in color images, which was the research focus of Chapter 3. This framework integrated the principal component analysis with a newly introduced mean-shift decomposition technique that resulted in key analyses of the inverse-intensity chromaticity space and in subtle but powerful variations of its dynamic range. This approach relies mainly on image reflectance correlations in defining a suitable linear expression that separates a scene image into two distinct sets of image reflectance representing the diffuse and specular image pixels. As structured, the approach described relies solely on image color information of dichromatic surfaces without requiring segmentation procedures, which is viewed here as a major contribution.

The results in applying this mathematical framework for the separation of specular and diffuse reflectance in color images clearly reveal distinct characteristics of the specular and the diffuse pixels, which only add credence to the merit of this new method. More importantly, Chapter 3 proved that the method used for iteratively eroding the specular component at each pixel shows a realistic outcome that even in the histogram of these pixels intensity distribution, one could see a continuity akin to a same real-world object but without its specular content. In other words the rendering after removal of the specular component is true like. In fact, it was almost astounding how natural the Specular Removal process seemed when a cross section of a given image is observed (i.e., the color distribution in a given image line that goes through the diffuse and into the 
image part where the specular part has been removed). This natural rendering is maintained as was proven in all three channels (Red, Green and Blue).

Furthermore, a scene illumination rendering technique based on the specular/diffuse separation concept was newly developed solely based on local color information, The results obtained showed that the suggested method renders any type of scenes with surfaces represented by the dichromatic reflectance model. These results serve as additional evidence to the merits of the Mean-Shift Decomposition technique that was devised to separate the image reflection components based on the image chromaticity constituent distribution in the Inverse-Intensity Chromaticity Space. These results were obtained while still maintaining the inner intrinsic image components unmarked, contributing significantly to the realistic renderings that were accomplished.

This research endeavor also showed that by remodeling the shape of the specular reflection cluster, it became possible to render the illumination attributes characterizing any illuminated scene image. Finally, the possibility is also given for rendering different illumination effects depicting real-world lighting conditions.

In view of these findings, this dissertation also laid out the framework for a novel color constancy method based on the dichromatic reflectance model. This showed that by extracting the most prominent reflectance components in a scene image, the proposed method was able to determine a good approximation of the canonical illuminant. The MSD introduced in chapter III was again used as an integrated module to decompose the image reflectance components into two distinct clusters, one representing the diffuse reflectance distribution and second representing the specular reflectance distribution. In addition, the specular reflectance components were evaluated using an innovative voting 
scheme to extract the most dominant reflectance component reflecting the true illuminant chromaticity. This small set of reflectance was then analyzed to find the reflectance components that correspond most to the illumination color. Experimental results show a good improvement over the most used color constancy methods found in literature.

Recall that in the axis transformation that was to facilitate the linearization of the reflection data stretching process needed for illumination modeling, thresholds to remodel the specular component values. This process was important in order to achieve the desired rendering effect. Although the results were near ideal, the threshold values were approximated to render each input image channels of the white reference patch. This simulates the effects generated by the real-illuminants (as shown in the estimated image column). It would have been even more appealing if such thresholds were mathematically generated on the basis of the inverse-intensity chromaticity space, a space that was used to analyze the relationship between illumination color and image intensity. Furthermore, another important issue that was not fully resolved in this dissertation is the definition of the exact distance that estimates the shifting interval between each specular element and its new diffuse value, an important step for better diffuse reflectance estimation.

Another important step that needs further consideration is the exploration of applications where specular illumination reflections are restricted. Different chromaticity based decomposition methods need to be investigated more thoroughly to accurately disassociate both reflections components for this kind of illumination situations. 


\section{LIST OF REFERENCES}

Arici, T., Dikbas, S., Altunbasak, Y., "A Histogram Modification Framework and Its Application for Image Contrast Enhancement", IEEE Transactions onImage Processing, vol.18, no.9, pp.1921-1935, 2009.

Bajcsy R., Lee S., and Leonardis A., "Detection of diffuse and specular interface reflections and inter-reflections by color image segmentation", International Journal of Computer Vision, 17(3):241.272, 1996.

Barnard, K., Martin, L., and Funt, B. V., "Colour by correlation in three-dimensional colour space". In Proceeding of the $6^{\text {th }}$ European Conference on Computer Vision, pp.375-389, 2000.

Barnard K., Martin L., Funt B., and Coath A., "A Data Set for Colour Research", Color Research and Application, Volume 27, Number 3, pp. 147-151, 2002.

Barnard K.,Ciurea F., and Funt B.,"Sensor sharpening for computational color constancy". JOSA, 18(11): 2728-2743, 2001.

Brainard, D. H., Delahunt, P. B., Freeman, W. T., Kraft, J. M., and Xiao, B., "Bayesian model of human color constancy", Journal of Vision 6, 11, pp. 1267-1281, 2006.

Brainard, D. H., and Freeman, W. T., "Bayesian color constancy", Journal of Optical Society of America A, 14, 7, pp.1393-1411, 1997.

Brooks, M., and Horn, B., "Shape and source from shading", In MIT AI Memo, 1985.

Chakrabarti, A. and Hirakawa, K. and Zickler, T., "Computational Color Constancy with Spatial Correlations", Harvard School of Engineering and Applied Sciences, TR-09$10,2010$.

Cheng Y., "Mean shift, mode seeking, and clustering", IEEE Transactions onPattern Analysis and Machine Intelligence, vol.17, no.8, pp.790-799, Aug 1995.

Chong, H. Y., Gortler, S. J. and Zickler, T., "The von kries hypothesis and a basis for color constancy", IEEE International Conference on Computer Vision, 2007.

Ciurea, F., Funt, B., "A large image database for color constancy research", Proceeding of IS\&T/SID’s Color Imaging Conference, pp. 160-64, 2004.

Ebner M., "Color constancy using local color shifts", IEEE European Conference on Computer Vision, 276-287. 2004.

Fairchild, M. D., "Color Appearance Models", Wiley-IS\&T Series in Imaging Science and Technology, 2005. 
Finlayson, G. D., Hordley, S. D., and Drew, M. S., "Removing shadows from images", IEEE European Conference on Computer Vision, pp.823-836, 2002.

Finlayson, G.D., Drew, M.S., Funt, B.V., "Spectral sharpening: sensor transformations for improved color constancy”, JOSA.11(4), 1553-1563, 1994.

Finlayson, G.D., Funt, B.V., "Coefficient channels: Derivation and relationship to other theoretical studies", Color Res. Appl. 21(2), 87-96, 1996.

Finlayson, G. D., Hordley, S. D., Hubel, P. M., "Color by correlation: a simple unifying framework for color constancy", IEEE Transactions on Pattern Analysis and Machine Intelligence, vol. 23, no.11, pp. 1209-1221, 2001.

Finlayson, G. D., Hordley, S. D., and Tastl, I., "Gamut constrained illuminant estimation”, International Journal of Computer Vision, vol. 67, no. 1, pp.93-109, 2006.

Finlayson, G.D., and Schaefer, G., "Solving for color constancy using a constrained dichromatic reflection model", International Journal ofComputer Vision, 42(3): 127-144, 2001 .

Finlayson, G., and Trezzi, E., "Shades of gray and colour constancy", IS\&T/SID Twelfth Color Imaging Conference, pp. 37-41, 2004.

Gehler, P.V., Rother, C., Blake, A., Minka, T., Sharp, T., "Bayesian color constancy revisited", IEEE Conference on Computer Vision and Pattern Recognition, vol., no., pp.1-8, 23-28, 2008.

Gijsenij, A, Gevers, T. , "Color Constancy using Natural Image Statistics and Scene Semantics", IEEE Transactions onPattern Analysis and Machine Intelligence, 2010.

Huynh, C. P., and Robles-Kelly, A., "A Solution of the Dichromatic Model for Multispectral Photometric Invariance", International Journal of Computer Vision, Vol. 40(1), 1-27, 2010.

Ji, T. L., Sundareshan, M. K., Roehrig, H., “Adaptive image contrast enhancement based on human visual properties", IEEE Transactions onMedical Imaging, vol.13, no.4, pp.573-586, Dec 1994.

Kaufman, L., and Rousseeuw, P. J., "Finding Groups in Data: an Introduction to Cluster Analysis", Wiley, New York, 1990.

Kawakami R.,Tan T. R., Ikeuchi K., "Consistent surface color for texturing large objects in outdoor scenes", IEEE International Conference on Computer Vision, Vol.2, pp.1200-1207, Beijing, China, Oct. 2005.

Klinker, G.J., Shafer, S.A., and Kanade, T., “The Measurement of Highlights in Color 
Images", International Journal of Computer Vision, vol. 2, pp. 7- 32, 1990.

Lee, H. C., Breneman, E. J., and Schulte, C. P., "Modeling light reflection for computer color vision", IEEE Transactions on Pattern Analysis and Machine Intelligence, 12(4), 402-409, 1990.

Lee, H. C., "Method for computing the scene-illuminant from specular highlights", Journal of Optics Society of America A., 3(10): 1694-1699, 1986.

Lin, S., Li, Y., Kang, S.B., Tong, X. and Shum, H.Y., "Diffuse-Specular Separation and Depth Recovery from Image Sequences", Proc. European Conf. Computer Vision, pp. 210-224, 2002.

Lin, S., and Shum, H.Y., "Separation of Diffuse and Specular Reflection in Color Images", Proc. IEEE Conf. Computer Vision and Pattern Recognition, 2001.

Mallick, S. P., Zickler, T., Belhumeur, P. N., Kriegman, D. J., "Specularity removal in images and videos: a PDE approach", IEEE European Conference on Computer Vision, 2006.

Mallick, S., Zickler, T., Kriegman, D., and Belhumeur, P., "Beyond lambert: Reconstructing specular surfaces using colors", In IEEE conference on Computer Vision and Pattern Recognition, II: 619-626, 2005.

Nayar, S.K., Fang, X.S., and Boult, T., "Separation of Reflection Components Using Color and Polarization", International Journal of Computer Vision, vol. 21, no. 3, 1996.

Nishino, K., Sato, Y., Ikeuchi, K., "Eigen-Texture method: appearance compression and synthesis based on a 3D model", Pattern Analysis and Machine Intelligence, IEEE Transactions on, vol.23, no.11, pp.1257-1265, Nov 2001.

Park, J. W., Lee, K. H., "Inpainting highlights using color line projection", IEICE Transactions on Information Systems, E90D: 250-257, 2007.

Parkkinen, J.P.S., Hallikainen, J., and Jasskelainen, T., "Characteristic Spectra of Munsell Colors", J. Optics Soc. Am. A, vol. 6, 1989.

Ping, T., Lin S., Quan L., and Shum H. Y., "Highlight removal by illuminationconstrained inpainting", In ICCV, pages 164.169, Nice, France, 2003.

Planck M., "The theory of heat radiation”, Dover Publication, NY, 1959.

Rahman Z., Jobson J. D., and Woodell A. G., "Retinex processing for automatic image enhancement", J. Electron. Imaging 13, 100, 2004.

Sato, Y., and Ikeuchi, K., "Temporal-Color Space Analysis of Reflection”, J. Optics 
Soc. Am. A, vol. 11, 1994.

Shafer, S., "Using color to separate reflection components", Color research and applications, 10(4): 210-218, 1985.

Storring, M., Andersen, H.J., Granum, E., "Estimation of the illuminant colour from human skin colour", Proceedings of the fourth IEEE International Conference on Automatic Face and Gesture Recognition, pp.64-69, 2000.

Tan, R. T., and Ikeuchi, K., "Separating reflection components of textured surfaces using a single image", IEEE Transactions on Pattern Analysis and Machine Intelligence, 27: 178-193, 2005.

Tan, R. T., Nishino, K., and Ikeuchi, K., "Separating reflection components based on chromaticity and noise analysis", IEEE Transactions on Pattern Analysis and Machine Intelligence, 26: 1373-1979, 2004.

Tan, R. T., Nishino, K., and Ikeuchi, K., "Color constancy through inverse-intensity chromaticity space", Journal of the Optical Society of America A. 21(3), pp.321-334, 2004b.

Tominaga, S., and Moruchi, Y., "Principal Component Analysis-Based Reflectance Analysis/ Synthesis of Cosmetic Foundation", The Journal of Imaging Science and Technology.vol. 53(6), 2009.

Tominaga, S., and Wandell, B. A., "Standard surface-reflectance model and illumination estimation", Journal of Optics Society of America A., 6(4): 576-584, 1989.

Wolff, L.B., and Boult, T., "Constraining Object Features Using Polarization Reflectance Model”, IEEE Trans. Pattern Analysis and Machine Intelligence, vol. 13, no. 7, pp. 635-657, July 1991.

Wyszecki, G., and Stiles, W.,"Color Science: concepts and methods, quantitative data and formulae", Wiley, 1982.

Yoon, K., and Kweon, I. S., "Correspondence search in the presence of specular highlights using specular-free two band images", In Proceedings of Asian Conference on Computer Vision, pp. 761-770, 2006.

Zickler, T., Mallick, P., and Kriegman, D. J., "Color subspaces as photometric invariants", International Journal ofComputer Vision, 79: 13-30, 2008. 
VITA

MOUNCEF LAHLOU

$\begin{array}{ll}1981 & \text { Born, Casablanca, Morocco } \\ 2002 & \begin{array}{l}\text { Scientific CPGE } \\ \text { Euro American Institute of Technology, SKEMA } \\ \text { Sophia Antipolis, France }\end{array} \\ & \text { B.S., Computer Engineering } \\ & \text { Florida International University } \\ & \text { Miami, Florida } \\ & \\ & \text { M.S., Computer Engineering } \\ & \text { Florida International University } \\ & \text { Miami, Florida } \\ 2006 & \text { Doctoral Candidate, Electrical Engineering } \\ & \begin{array}{l}\text { Florida International University } \\ \text { Miami, Florida }\end{array}\end{array}$

\begin{abstract}
AWARDS
International Student Scholarship, E.A.I. Tech, 2000 - 2001.

International Student Tuition Waver Award, FIU, 2004.

Graduate Student Research Assistantship, CATE/FIU, 2005 - 2010.

Dissertation Year Fellowship, FIU, 2010 - 2011.
\end{abstract}

\title{
PUBLICATIONS AND PRESENTATIONS
}

1. Lahlou, M., and Adjouadi, M., "Color based specular reflectance estimation for scene illumination rendering”. Journal of Vision, Submitted, 2011.

2. Lahlou, M., and Adjouadi, M., "Surface reflectance components separation from single image using the mean-shift-decomposition technique". International Journal of Innovative Computing, Information and Control, submitted, 2011. 
3. Lahlou, M., and Adjouadi, M., "Color constancy based dichromatic objects reflectance decomposition". IEEE Transactions on Image Processing, to be submitted, 2011.

4. Lahlou, M., and Adjouadi, M., "Color based specular reflectance estimation for scene illumination rendering". Computer Graphics International (CGI), accepted, 2011.

5. Lahlou, M., Adjouadi, M., and Barreto, A., "A Robust Face Detection Method Using Intrinsic Images and an Energy Minimization Technique under the NearIR light Spectrum", The 2009 International Conference on Image Processing, Computer Vision, and Pattern Recognition, Vol. I, pp. 351-356, July 2009.

6. Lahlou, M., Adjouadi, M., and Barreto, A., "Illumination Invariant Face Detection with Time-Dependent Intrinsic Images under Near-IR lighting", Proceedings of the CAHSI Annual Meeting, pp. 26-29, Google headquarters, Mountain View, CA, Jan 2009.

7. Lahlou, M., and Adjouadi, M., "Human-Computer Interface Design for Cognitive Workload Detection Using Pupillary Response", pp. 46-48, Proceedings of the 2nd Annual CAHSI Workshop, Miami, FL, Dec 2007.

8. Delgado, J., Guillen, M. R., Lahlou, M., Adjouadi, M., Barreto, A., and Rishe, N., "MIND: A Tiled Display, Visualization System at CATE/FIU", Proceedings of the IASTED International Conference on Graphics and Visualization in Engineering, pp. 68-73, ISBN 978-0-88986-67, Clearwater, FL, Jan 2007.

9. Lahlou, M., Guillen, M. R., Adjouadi, M., and Gaillard, W. D., "MEDIAR: An Online Web-Based Repository site of fMRI Medical Images and clinical data for Childhood Epilepsy", 11th World Congress on Internet in Medicine, Ontario, Canada, October 13-20, Oct 2006.

10. "Application of Nonlinear Classifiers with PCA in fMRI Language Activation Pattern Recognition in Multisite Study for Pediatric Epilepsy". International Conference on Image Processing, Computer Vision, and Pattern Recognition, IPCV, Las Vegas, NV, July 2009.

11. "Modified Fast Principle Component Analysis". International Conference on Image Processing, Computer Vision, and Pattern Recognition, IPCV, Las Vegas, NV, July 2009.

12. "A Robust Face Detection Method Using Intrinsic Images and an Energy Minimization Technique under the Near-IR Light Spectrum". IPCV, Las Vegas, NV, July 2009. 